Science and Engineering Research Council

Rutherford Appleton Laboratory

Chilton DIDCOT Oxon OX11 0QX

RAL-90-077

***** RAI IIBARI R6I ****

ACC_No: 208462

Shelf: RAL 90077

\title{
Physics and Chemistry of materials
} from Neutron Diffraction and Spectroscopy

Ulf Dahlborg and Stephen W. Lovesey

November 1990

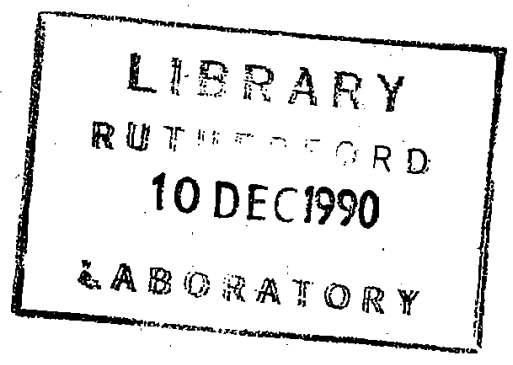


Science and Engineering Research Council "The Science and Engineering Research Council does not accept any responsibility for loss or damage arising from the use of information contained in any of its reports or in any communication about its tests or investigations" 


\title{
PHYSICS AND CHEMISTRY OF MATERIALS FROM
}

\section{NEUTRON DIFFRACTION AND SPECTROSCOPY}

\author{
by \\ ULF DAHLBORG \\ Institute of Physics, \\ Department of Neutron and Reactor Physics, \\ Royal Institute of Technology, S-100 44, Stockholm, Sweden \\ and \\ STEPHEN W. LOVESEY \\ Rutherford Appleton Laboratory, \\ Oxfordshire, OX11 0QX, England \\ and \\ Institute of Physics, \\ Uppsala University, S-751 21, Uppsala, Sweden
}



Abstract

A short introduction to the powerful techniques of neutron diffraction and spectroscopy is illustrated largely with achievements by Swedish researchers in the past few years. Background material on sources and instrumentation is included, together with a directory of facilities routinely available to the Swedish scientific community. 


\section{Prologue}

Scattering experiments are important in many branches of chemistry and physics research. In condensed matter and materials research the projectiles used include atomic ions, electrons, neutrons and photons. While some experiments, like laser spectroscopy and $x$-ray diffraction, require a modest financial outlay, most demand access to major experimental facilities. The construction and operational costs are such that there is a trend toward central domestic and multi-national facilities, e.g. within Europe there is the neutron reactor source at the Institut Laue-Langevin, and the European Synchrotron Radiation Facility under construction next door to the ILL in Grenoble.

Multi-national facilities are often particularly well-funded and provide advanced instrumentation, but over-demand frequently forces discrimination against timeconsuming complex experiments and training exercises. These are two of the major reasons why, more modest, domestic facilities like the R2 reactor at Studsvik are essential. Another is that optimal use of multi-national facilities is only possible initial feasibility studies. Very often the information obtainable from a neutron scattering experiment is not available from other experimental techniques. The host of valuable and unique features of the neutron scattering technique vindicate the cost of building and operating neutron beam facilities.

Neutron diffraction studies of crystals and disordered systems provide structural information which complements $x$-ray results since, for non-magnetic materials, neutrons scatter predominantly from nuclei. The strength of this scattering varies quite strongly from one isotope to isotope, in an almost random manner, so contrast studies using isotopic substitution are widely used and particularly with hydrogenous materials. Magnetic diffraction provides information on both the configuration and distribution of the magnetization density. Neutron spectroscopy is widely used for the determination of; (a) dispersion curves of collective excitations in crystals (phonons), liquids and magnetic materials (spin waves); (b) localized, non-dispersive excitations such as hydrogen vibrational states in hydrides and macromolecules and atomic and crystal field states in magnetic materials; (c) diffusion of protons and (d) proton motions in 
macromolecular (polymer) systems.

The intention of this paper is two-fold. First, to serve as a brief introduction to neutron scattering (diffraction and spectroscopy) in investigations of properties of matter on an atomic scale. Emphasis is placed on results deduced from the interpretation of experiments rather than experimental methods, instrumentation, and data analysis. In view of the limited space available, an attempt is made to reference recent review articles, books and conference proceedings.

Secondly, the aim is to provide a perspective of Swedish neutron scattering work in chemistry and physics, and the facilities currently available to the Swedish science community. To this end, access to instrumentation on the $\mathrm{R} 2$ reactor at Studsvik, and the pulsed neutron facility ISIS at the Rutherford Appleton Laboratory, is described in an annex together with cameos of various spectrometers. Here again, the information we can provide is necessarily limited. The reader interested in performing experiments should consult the reference manuals listed and one of the personnel named in the annex.

In many respects the Swedish scientific community is privileged as regards access to neutron scattering facilities. The dornestic reactor facility at Studsvik has recently been refurbished with large beam tubes. By the end of 1990 there will be a suite of instruments suitable for a range of diffraction and spectroscopic studies of crystals, powders, amorphous materials and liquids. Instrumentation on the ISIS facility is largely complementary, giving access to large ranges of energies and wavelengths. The very large signal-to-rioise ratio available on ISIS instruments means that the resolution attainable in several cases is unmatched by similar instruments placed at reactors.

The new user can draw on a store of experience held by Swedish scientists who have worked at the forefront of neutron scattering research. Admittedly the experience is predominantly with nuclear scattering, but studies of magnetic phenomena are now planned.

Basic properties of the neutron and a sketch of its discovery are provided in sections 2 and 3 , respectively. A broad based review, which includes a listing of neutron 
properties, historical notes and a survey of techniques and sources has been prepared by Scherm [1]. Here, neutron sources are briefly reviewed in section 4 and the basic concepts required for the interpretation of experiments are outlined in section 5 . Theoretical work is underpined by the framework of linear response theory [2], which acts also as a unifying language with the interpretation of many other experiments such as NMR, Mössbauer, $\mu \mathrm{SR}$, and light scattering [2]. Illustrative examples of neutron beam research, with a strong slant towards work involving Swedish scientists and facilities, are provided in section 6 . In view of this selection the examples are mostly studies in which nuclear scattering is used. However, the material in section 5 on magnetic scattering should hopefully give the reader an inkling as to the power of neutron scattering in studies of magnetic phenomena. Reviews of the relevant theory and experiments can be found in references $[2,3,4]$. Our speculation on future trends in neutron beam research is given in section 7 .

\section{Neutron Properties}

The utility of neutron scattering as an atomic-scale probe of condensed matter stems from the relative weakness of neutron-matter scattering, the compatibility of neutron energies and wavelengths with characteristic energies and lengths of atomic motions in solids and liquids, and the magnetic moment of the neutron. The neutron-matter interaction is so weak that first-order perturbation theory is wholly adequate to account for the neutron scattering cross-section. In other words, neutron scattering provides information on the chemical and physical properties of matter that is undistorted by the radiation. Furthermore, the interpretation of the measured cross-section is not clouded by uncertainty about the nature of the radiation-matter interaction or specification of the cross-section.

Neutrons interact with the nuclei and electrons in matter, and the scattering crosssections are similar in magnitude. It is possible to discriminate between scattering events involving nuclei and electrons. In consequence, the neutron scattering technique provides unambiguous information on the positions and motions both of nuclei and of electrons. Moreover, since neutrons penetrate deeply into matter they provide an ideal probe of the bulk properties of matter [1-4]. However, neutrons are also absorbed in matter. 
Neutron nuclear scattering as well as the absorption cross sections vary from one isotope to isotope in a more or less random manner. In many applications it is particularly advantageous that the scattering cross sections for the proton and deuteron are very different. The cross section for a proton is a massive 82 barns ( 1 barn $=10^{-24}$ $\mathrm{cm}^{2}$ ) whereas for a deuteron it is an order of magnitude smaller. Hence, the proton function in macromolecules, membranes, etc, is readily studied, while deuteration enables us to pick out properties of the host environment. The magnitude of neutron scattering and absorption cross sections for some selected nuclei are given in table 1.

The energy $E$ of a neutron with a wave vector $k$ is

$$
E=\hbar^{2} k^{2} / 2 m
$$

where $k=|k|$ and $m$ is the neutron mass. Energies are often given in units of $m e V=$ $10^{-3} \mathrm{eV}$, and $\left(\hbar^{2} / 2 \mathrm{~m}\right)=2.08 \mathrm{meV} \AA^{2}$. In terms of the neutron wavelength $\lambda$ we have,

$$
\lambda=\left(h^{2} / 2 \mathrm{mE}\right)^{1 / 2}=9.04 \mathrm{E}^{-1 / 2},
$$

where $\lambda$ is in $A$ and $E$ is in meV. Here we have chosen the energy unit favoured by physicists. Other energy units frequently used in spectroscopy are related to the meV through

$$
1 \mathrm{meV} \equiv 0.24 \mathrm{THz} \equiv 8.07 \mathrm{~cm}^{-1} \equiv 11.61 \mathrm{~K}
$$

and the conversion to temperature is included for completeness.

It is perhaps useful at this junction to recall that in contrast to neutron scattering from materials photon scattering is dominated by the photon-electron interaction, described by quantum electrodynamics. Absorption of photons due to the photo-electric process and Compton effect severely reduce photon penetration in matter, and so the technique is highly surface sensitive. The photon-matter interaction contains complicated processes, some of which involve nonlinear events not describable within the framework of linear reponse theory that underpins the interpretation of neutron scattering (excluding events that involve compound nuclear resonance states). 
The high intensity of photon beams from synchrotron sources makes it feasible to exploit magnetic photon scattering ( a relativistic correction to the Thompson amplitude) as a probe of condensed matter [5]. This technique has some advantages with respect to magnetic neutron scattering, although it is likely to be confined to elastic (diffraction) and Compton scattering studies for the most part in the near future.

By and large, selection rules, which are manifestations of high symmetry conditions, operate more forcefully in photon scattering than in neutron scattering. In optical spectroscopy, for example, the change in photon wave vector, $\mathbf{k}$, is so small that events obey the dipole selection rule, whereas in a corresponding neutron induced event $\mathbf{k}$ can be relatively large and additional processes are engaged, c.f. section 5.2. Similar reasoning holds for the excitation of states in a crystal. Selection rules are manifest at points of high symmetry in the Brillouin zone, such as the zone centre and boundary, and generally absent at an arbitrary point on a dispersion curve.

\section{History}

Joliot-Curie's communication of 28 January 1932, reported that alleged $\gamma$-rays from the $\alpha$-beryllium reaction were capable of ejecting protons from paraffin. When the paper reached Chadwick, at the Cavendish Laboratory, he went to work and on 17 February submitted a paper entitled 'possible existence of a neutron' in which he proposed that the $\alpha$-beryllium reaction is $\alpha+\mathrm{Be}^{9}=\mathrm{C}^{12}+\mathrm{n}$. Chadwick's discovery of the neutron, in a few days of strenuous work, concluded a search that, off and on, had been conducted at the Cavendish for more than a decade.

In early 1934 Fermi submitted the first in a series of articles on radioactivity induced by neutron bombardment. With this paper Fermi started experimental studies in neutron physics that made him perhaps the world's leading authority on the subject during the nineteen thirties. His pseudopotential method, applied to neutron scattering by nuclei in condensed matter, appeared in 1936 and it remains the cornerstone of the interpretation of low energy neutron-nucleus scattering experiments.

In the same year Bloch predicted that the electromagnetic neutron-electron amplitude is similar in magnitude to the classical electron radius, and therefore comparable to 
ruclear scattering amplitudes. The following year Schwinger queried Bloch's calculation: the latter is now known to be incorrect, and Schwinger provides the correct result although his reasoning is erroneous. A correct calculation, and physical interpretation, is provided by Migdal in a paper submitted in July 1938 to a Russian Journal. However, Migdal's work was unnoticed in the West, and the Bloch-Schwinger controversy was finally settled in 1951 when two independent experiments found unambiguous evidence to support the result given by Schwinger. The basis of the interpretation of neutronelectron scattering experiments is the paper by Halpern and Johnson published in 1939. (Although these authors subscribe to the Schwinger view they nevertheless propose experiments to settle the Bloch-Schwinger controversy.)

The major sources of neutrons in the early nineteen thirties were radium-beryllium sources. Even though it was possible to demonstrate the diffractive properties of neutrons, the low intensities from radium-beryllium did not permit the practical use of neutron beams to study the properties of condensed matter. The latter began to flourish with the development of nuclear reactors. The Oak Ridge Graphite Reactor and the CP-3 reactor at the Argonne National Laboratory became operational in 1943 and 1944 , respectively.

Neutron intensities produced by modern, high-flux reactors are three orders of magritude larger that those obtained with the early reactors at the Oak Ridge and Argorıne Laboratories. Moreover, new vistas are now being opened with advanced spallation sources which utilize protons, accelerated in a synchrotron, to liberate a very large supply of energetic neutrons from heavy metal targets.

\section{Neutron Sources}

The self-sustaining nuclear fission reaction has been used as the basis for most neutron sources primarily constructed for scattering experimerits. The effective thermal neutron fluxes available from steady-state reactors has steadily improved over the past $\mathbf{4 0}$ years, and a saturation level has been reached at approximately $1.2 \times 10^{15} \mathrm{n} / \mathrm{cm}^{2} \mathrm{~s}$, the flux provided by the high-flux reactor at the institut Laue Langevin (ILL), Grenoble. 
The practical limits in this flux are imposed by a combination of engineering constraints, due to the large power density in the core, as well as to economic factors. Plans are underway to construct a new high flux reactor at the Oak Ridge National Laboratory, USA, which has a flux increase of the order 2-4 over the ILL reactor; this will require the solution of new technological problems and an investment in the region of 2000 MSEK. Future improvements at existing reactor sources will result from upgrading their power and their associated experimental facilities, and a review of current proposals has been published by Moon and West [6]. It is nevertheless generally agreed that the practically attainable flux from a steady state reactor has reached its limit.

Pulsed neutron sources are able to provide instantaneous neutron fluxes which are higher than those from continuous reactors. Since they only operate for a fraction of a time period, their momentary power densities and neutron fluxes can be very high, while their average power densities can be kept at modest levels compatible with ease of heat extraction. Practical pulsed sources for neutron scattering can be either pulsed reactors or accelerator-based systems in which the burst of fast (MeV) neutrons is moderated in a hydrogenous moderator to provide the effective source for the experiments. The proton spallation process is the most competitive accelerator-based neutron source to date; it has the highest neutron yield per incident particle and also the lowest energy dissipation in the target per neutron generated. Typical values of these parameters for the most frequently used neutron-producing reactions are summarized in table 2 together with corresponding target powers for existing sources.

An important parameter, perhaps most relevent one when discussing the possibilities to remove energy in a neutron-producing facility, is the power density. For the ILL reactor this is about $600 \mathrm{~kW} / \mathrm{l}$ while the corresponding figure for ISIS is about $75 \mathrm{~kW} /$ which indicates that the spallation sources by no means have reached its maximum strength with the advent of ISIS: A pulsed beam imposes, however, a higher demand on the efficiency of energy removal.

As a final topic in this section we mention that a pulsed reactor has been constructed at Dubna, USSR. Fission occurs during the short time that a rotating reflector blade (rotational frequency $5 \mathrm{~Hz}$ ) passes $\mathrm{a}^{239} \mathrm{PuO}_{2}$ target, and the average power generated is approximately $4 \mathrm{MW}$ giving an impressive instantaneous thermal neutron flux of order 
$10^{16} \mathrm{n} / \mathrm{cm}^{2} \mathrm{~s}$. The major disadvantage is the inherently long pulse width (approximately $250 \mu$ s after moderation) so that only relatively poor resolution experiments are possible, particularly with short wavelength neutrons. There is also the potential hazard associated with moving substantial mechanical objects to a high degree of reliability in an intense radiation environment.

\section{Baslc Concepts}

Since the scattering of slow neutrons is a weak process it can be described by firstorder perturbation theory, i.e. Fermi's Golden Rule for transition rates. In consequence, we treat the incident and scattered neutron states as plane waves with energies $E, E^{\prime}$ and wave vectors $\mathbf{k}, \mathbf{k}^{\prime}$, related as in eqn (1). The cross-section, or response function, is described in a four dimensional space spanned by the variables

$$
\hbar \omega=E-E^{\prime}
$$

and

$$
\mathbf{Q}=\mathbf{k}-\mathbf{k}^{\prime}
$$

If the target sample is spatially isotropic the response function depends only on $\omega$ and $Q=|Q|$.

The differential cross-section, which has the dimension of (area/energy), is readily expressed in terms of correlation functions that are determined solely by the chemical and physical properties of the target sample. This is by far the most elegant and powerful representation, and the orie adopted here. Further details, including an exposition of linear response theory, are found in [2] and the theory chapters in $[3,4]$. We will separately describe nuclear and magnetic scattering, though some basic concepts are common to both, of course. Although polarization phenomena are important, and more widely used today with improved sources and instrumentation, we do not include it in the basic theory because this is intended very much as an introduction and therefore it should be kept as simple as is reasonably possible. Full accounts of polarization phenomena are provided in references $[2,7]$. 


\subsection{Nuclear Scattering}

Slow neutron scattering is described by a single parameter, namely a scattering length $b$ which is assumed to have minimal energy dependence. The imaginary part of $b$ gives rise to absorption. This is extremely large for some isotopes, e.g. about 15000 barns for $\mathrm{He}^{3}$ and $5 \AA$ neutrons.

The nuclear scattering cross-section is a scattering length weighted sum of correlation functions. Let $i, j$ label the scattering nuclei, and denote the correlation function by $Y_{i j}(Q, t)$. With this notation the partial differential cross-section is,

$$
\frac{d^{2} \sigma}{d \Omega d E^{\prime}}=\left(E / E^{\prime}\right)^{1 / 2} \int d t(1 / 2 \pi \hbar) e^{-\mid \omega t} \sum_{1, j} b_{1}{ }^{*} b_{j} Y_{\|}(Q, t),
$$

where $d \Omega$ is the solid angle subtended by the detector. The function $Y_{i j}(Q, t)$ possesses properties that make the right-hand side of (5) both real and either positive or zero, as required for a response function. It is necessary to average the cross-section over the distribution of nuclear spins (assumed to be completely random), isotopes, substitutional disorder, etc. We will deal with these averages as and when required.

5.1.1. Purely elastic scattering. This is generated by the value of the correlation function at infinite time; $Y_{1 \mathrm{j}}(Q, \infty)$ with $Q \neq 0$ is finite for crystals and fully arrested super-cooled liquids, for example, but vanishes for normal liquids. For a crystal, it is customary to write

$$
Y_{i j}(\mathbf{Q}, \infty)=\exp \left\{i Q \cdot\left(\mathbf{R}_{j}-\mathbf{R}_{i}\right)-W_{j}(\mathbf{Q})-W_{i}(\mathbf{Q})\right\}
$$

where $\mathbf{R}_{\mathbf{1}}$ is the equilibrium lattice position of the $\mathbf{i}^{\prime}$ th atom and the remaining terms are Debye-Waller factors. An explicit expression for $W(Q)$ valid for a harmonic lattice is given following eqn (16).

Bragg scattering is elastic and coherent and occurs under special geometrical conditions. It is generated by a perfect crystal, which means that the appropriate crosssection is formed with the square of the average of the effective scattering length per 
unit cell. Let the crystal contain $\mathrm{N}$ unit cells of volume $\mathrm{v}_{\mathrm{o}}$ in which the atoms are at sites defined by position vectors $\mathbf{d}$. The elastic coherent cross-section is then

$\left(\frac{\mathrm{d} \sigma}{\mathrm{d} \Omega}\right)_{\text {coh }}^{\text {el }}=N(2 \pi)^{3} / v_{0} \sum_{\tau}\left|F_{N}(\tau)\right|^{2} \delta(Q-\tau)$

in which $\{\tau\}$ are reciprocal lattice vectors and the unit cell structure factor

$F_{N}(Q)=\sum_{d} \bar{b}_{d} \exp \left(i Q . d-W_{d}(Q)\right)$

where $\bar{b}$ is the scattering length averaged over isotope and nuclear spin distributions. From (7) it is evident that scattering occurs only when the condition $\tau=Q$ is satisfied, which is a statement of Bragg's Law.

The difference between the total elastic scattering and the Bragg intensity frorn a solid is due to disorder and defects in the crystal, nuclear spins and mixtures of isotopes. By definition it is not coherent and it occurs to some extent at all scattering angles. It is customary, but not completely logical, to use the term incoherent scattering for nonBragg scattering generated by nuclear spins and isotope mixtures (note that a sample of a pure isotope can produce incoherent scattering if the nuclear spin is finite, e.g. $\mathrm{He}^{3}$ ). All the remaining non-Bragg scattering is called diffuse scattering.

Incoherent elastic scattering from a crystal measures the quantity,

$$
N \sum_{d}\left[\overline{b_{d}^{2}}-\left(\bar{b}_{d}\right)^{2}\right] \exp \left[-2 W_{d}(Q)\right]
$$

This can be interpreted as the sum over atoms in the unit cell of the Debye-Waller factor weighted by the mean-square fluctuation in the scattering length.

As an example of elastic diffuse scattering consider a binary system in which type-2 atoms (impurity atoms, say) occur with concentration $c$. All other things being equal apart from a difference in coherent scattering lengths, diffuse scattering occurs which is proportional to, 
$\left(\bar{b}_{2}-\bar{b}_{1}\right)^{2} c(1-c)$

A more realistic model would allow for the difference in Debye-Waller factors and the deformation in the host lattice created by the impurity atoms. In the latter case, the appropriate cross-section is proportional to the absolute square of the spatial Fourier transform of the deformation, which can be compared to theoretical predictions.

5.1.2. Total scattering. For a liquid strictly elastic events occur only if $\mathbf{Q}=0$, which corresponds to no scattering. Hence, the total scattering is measured in a neutron scattering experiment without energy analysis. This quantity is thus the response function integrated over all neutron energy transfers. With a monatomic sample, in which quantum effects are negligible (achieved with large $A$ atoms at relatively high temperatures), the total coherent scattering, at constant $Q$, is proportional to the structure factor,

$S(Q)=1+\rho_{0} \int d r e^{i Q r}[g(r)-1]$

in which $\rho_{0}$ is the particle density and $g(r)$ is the pair distribution function. It is, perhaps, useful to note that $r^{2} g(r)$ is the probability distribution for the particle density about the origin. Hence, the number of particles within a sphere of radius $R$ prescribed about a given particle is

$$
4 \pi \rho_{0} \int_{0}^{R} d r r^{2} g(r)
$$

In the limit $R \rightarrow \infty$ this quantity approaches the total number of atoms in the sample, as required.

Before turning to inelastic scattering we draw attention to a basic difference between Bragg scattering and total scattering. The latter is readily shown to be proportional to $Y_{i j}(Q, 0)$, i.e. the instantaneous value of the correlation function. On the other hand, Bragg scattering is proportional to the square of a time-averaged variable (a basic principle in statistical physics is that statistical averaging is completely equivalent to time averaging). The difference between these two extreme limits of the correlation function is related to the appropriate isothermal susceptibility, i.e. the mean-square fluctuation 
in the number density. We conclude that the difference is small except in the vicinity of a phase transition, when fluctuations take macroscopic values. One final point to make is that total scattering is often referred to as the static approximation to the crosssection, the choice of terminology being more or less self-evident in view of what we have just said.

5.1.3. Neutron spectroscopy. This corresponds to scattering events for which $\omega \neq 0$ and thus are termed inelastic. It is customary to use the label quasi-elastic for the part of the inelastic spectrum which arises from random, or stochastic, processes that occur over relatively long time scales [8], e.g. diffusive motion of an atom in a liquid. If classical statistics apply, the incoherent (single-particle) scattering cross-section is in this case determined by the correlation function

$$
Y(Q, t) \sim \exp \left(-Q^{2} D|t|\right)
$$

where $D$ is the self-diffusion constant. The corresponding cross-section is

$$
\frac{d^{2} \sigma}{d \Omega d E^{\prime}}=\left(\frac{E}{E^{\prime}}\right)^{1 / 2} \frac{\sigma_{1}}{4 \pi^{2}} \frac{Q^{2} D}{\omega^{2}+\left(Q^{2} D\right)^{2}}
$$

in which $\sigma_{1}$ is the incoherent cross-section (79.8 barns and 2.0 barris for a proton and deuteron, respectively). A similar result holds for a particle jumping between interstitial sites of a lattice, e.g. hydrogen diffusion in metals. In this instance $D=\ell^{2} / \tau$, where $\ell$ is a length, of order the lattice constant, and $\tau$ is the residence time at a given site.

The generic form of the correlation function that appears in quasi-elastic scattering is, to a good approximation,

$$
Y(Q, t)=\exp \left(-Q^{2} \mu(t) / 2\right)
$$

in which $3 \mu(t)$ is the mean-square displacement after a time $t$. The result $\mu(t)-|t|$ found for uncorrelated jumps on a lattice is characteristic of a random-walk process.

If a particle is bound in a crystal or macromolecule then often a useful starting point is to consider scattering from a harmonically bound particle. The scattering response for a particle with a natural frequency $\omega_{0}$ is, 
$\exp [-2 W(Q)+(1 / 2) \hbar \omega B] \sum_{n=-\infty}^{\infty} l_{n}(y) \delta\left[\hbar \omega-n \hbar \omega_{0}\right]$

The interpretation of (16) is straightforward; the scattering vanishes unless $\omega=n \omega_{0}$, and the integer $n$ measures the number of units of energy $\hbar \omega_{0}$ lost $(n>0)$ or gained $(n<0)$ by the neutron. The various quantities in (16) are; $B=1 / k_{B} T$ ( $T$ is the absolute temperature), $I_{n}(y)$ is a Bessel function of the first kind, and using the dimensionless variables $x=\left(\hbar \omega_{0} B / 2\right)$ and $\gamma=\left(\hbar Q^{2} / 2 M \omega_{0}\right)$ where $M$ is the mass of the scattering particle, $y=\gamma / \sinh (x)$ and $W=(\gamma / 2) \operatorname{coth}(x)$. Note that the elastic contribution $(n=0)$ contains $I_{0}(y)$. This factor arises from thermal fluctuations of the bound particle, which are negligible if the particle participates in a bulk collective motion.

The terms in (16) with $n= \pm 1$ are usually labelled the fundamental modes. If $y<<1$, as is often the case, the intensity of higher-order modes is very small in comparison with the fundamental since,

$$
I_{n}(y) \sim(1 / n !) y^{|n|} ; y \rightarrow 0
$$

In the limit $\left(\hbar Q^{2} / M \omega_{0}\right) \gg>1$, which can be achieved with a pulsed neutron source, it is not possible to select the fundamental mode in the response. A palisade of modes of near equal amplitude are engaged in scattering, so the expansion leading to the representation (16) is of minimal value. It can be shown that, in the limit of large $Q$, the scattering response tends to a Gaussian envelope function centred at the recoil energy $(\hbar Q)^{2} / 2 M$ with a mean-square width of $2 \omega_{0}{ }^{2} W(Q)$.

A far more realistic model of single particle dynamics is achieved by considering the particle as a defect in a host matrix. Two parameters characterize the particle matrix system, namely the ratio of the two masses and the deviation of the particle-matrix stretching force from that in the bulk matrix. The dynamics of the particle can be obtained in closed form if the stretching forces are harmonic. Calculations with this model reveal that a light mass (e.g. proton in a macromolecule) creates a high frequency mode which is well separated from the maximum phonon frequency in the pure host. By using a Debye model for the latter the frequency of the new mode is

$$
\omega=\left(0.6 \mathrm{M} / \mathrm{M}^{\prime}\right)^{1 / 2} \omega_{\mathrm{D}}
$$


where $\left(M / M^{\prime}\right)$ is the host-particle mass ratio, assumed to be large, and $\omega_{D}$ is the Debye frequency. This formula accounts for hydrogen mode frequencies in metal-hydrogen systems apart from those using palladium, which display other slightly unusual properties, (see section 6.4).

Detailed numerical calculations of the scattering response for a particle embedded in a matrix show that it contains a myriad of features [9]. For the two extreme cases of $Q \rightarrow 0$ and $Q \rightarrow \infty$ the response is well approximated by the fundamental contributions and a Gaussian envelope function, respectively. But, for intermediate $Q$ the line shapes are highly structured. Satellites to the harmonics appear which can be traced back to structure in the host density of states. Such features are understood by viewing the light mass particle as a probe of host lattice vibrations.

We turn now to scattering from collective atomic motions, or phonons. Neutron scattering is the established method of measuring phorion dispersion curves. To understand how this is possible consider the fundamental contribution to the harmonic oscillator response given in eqn. (16); this vanishes unless the neutron energy change matches the energy of one quantum. When scattering from a collective motion the energy selection-rule is supplemented by a wave vector selection-rule, $\mathbf{Q}=\mathbf{q}+\tau$ where $\mathbf{q}$ is the lattice wave vector (confined to a Brillouin zorie) which labels the phonon mode. In an experiment both $\mathbf{Q}$ and $\omega$ are determiried, and hence a point on the phonon dispersion curve is established.

The one-phonon scherne we have just described has no value in tackling the interpretation of coherent scattering from a liquid for which two extreme limits are well understood. For small $Q$ and $\omega$ we can appeal to linear hydrodynamics. The response for fixed $Q$ is found to consist of an elastic peak (Rayleigh line) and two inelastic peaks (Brillouin lines) at $\omega= \pm Q c_{0}$ where $c_{0}$ is the velocity of sound. In the opposite limit of large $Q$ the response resembles that of a free particle, namely a Guassian centred at the recoil energy $E_{R}=(\hbar Q)^{2} / 2 M$ with a mean-square width proportional to $\left(k_{B} T E_{R}\right)$. While much has been learnt about the nature of physical processes engaged at intermediate $Q$, the subject is on-going, particularly with regard to molecular liquids and the super-cooled state. 


\subsection{Magnetic Scattering}

Neutrons scatter off the magnetic field generated by unpaired electrons in a sample. Note that the number of electrons involved is generally a small fraction of those engaged in an $x$-ray experiment.

The magnetic field has two sources. First, the spins of electrons lead to a dipole-dipole interaction. Second, mobile electrons generate a field obtained from Biot-Savart's formula. In the forward direction $(Q \rightarrow 0)$ the neutron-electron interaction is simply related to the total magnetic moment. Hence, for an atom characterized by total spin and angular momentum $\mathbf{S}$ and $\mathbf{L}$, respectively, the forward scattering amplitude is generated by $(L+2 S)$.

The general form of the neutron-electron interaction for arbitrary $Q$ is quite complicated. Calculation of the matrix elements required in the interpretation of neutron-electron spectroscopy of isolated ions requires the full battery of Racah algebra used in atomic and nuclear spectroscopy. Even so, we can gain a good insight into the behaviour of magnetic neutron scattering by employing a relatively simple expression for the interaction, valid for modest values of $Q$.

For not too large $Q$, the magnetic interaction operator is

$$
r_{0}(1 / 2) g F(Q) J
$$

where the interaction strength $r_{0}=-0.54 .10^{-12} \mathrm{~cm}, \mathrm{gJ}$ is the magnetic moment operator and $F(Q)$ is an atomic form factor. For a spin-only ion $g J \rightarrow 2 S$, whereas for a rare earth ion $J=L+S$ and $g$ is the Lande' splitting factor. The form factor is defined to be unity in the forward direction, and decreases monotonically to a value of $\sim 0.2$ at $Q \sim 8$ $\AA^{-1}$ in a typical case. But, be warned, some form factors achieve a maximum value at a finite $Q[10]$.

The definitions of Bragg and total scattering carry over from the previous discussion of nuclear scattering. We begin our discussion with the total scattering from $N$ paramagnetic ions, namely, 


$$
\frac{d \sigma}{d \Omega}=N\left[\left(r_{d} / 2\right) g F(Q)\right]^{2}(2 / 3) J(J+1)
$$

where $J$ is the magnitude of the spin, i.e. $J \cdot J=J(J+1)$. This formula shows that the cross-section is large for large values of $\mathrm{J}$, just as we would expect. The dependence on the scattering vector $Q$ comes only through the form factor $F(Q)$.

There is, of course, a very strong $Q$ dependence in Bragg scattering from an ordered magnetic material. First, scattering vanishes unless $Q=\tau$ where $\{\tau\}$ are reciprocal lattice vectors for the magnetic structure. A second dependence arises from the fact that the component of $J$ perpendicular to $Q$ is observed. This feature often enables the moment orientation to be established. No such factor is explicit in (18) because the paramagnetic ions are randomly orientated. The Bragg cross-section for a collinear magnetic structure is

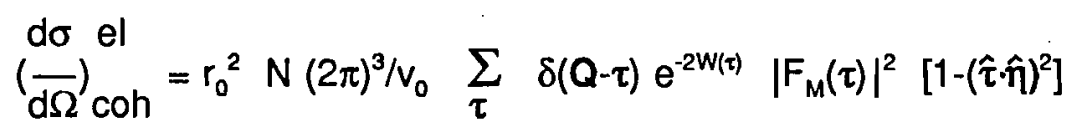

where $\hat{\eta}$ is a unit vector that defines the preferred magnetic (easy) axis and

$$
F_{M}(Q)=\sum_{d}(1 / 2) g_{d}<J>F_{d}(Q) \xi_{d} e^{l a d}
$$

in which $\xi_{d}= \pm 1$ according to the orientation of the magnetic moment relative to the preferred axis. Several features merit explicit mention. The total Bragg scattering from a magnetic material is described by the sum of (7) and (19). The moment, proportional to the thermal average $\langle J\rangle$, vanishes at the phase transition to the paramagnetic state. Bragg intensities can be used to obtain the critical exponent for the continuous decrease of the magnetization in the immediate vicinity of the transition. A magnetic material usually forms domains, in which case the orientation factor $\left\{1-(\hat{\tau} \hat{\eta})^{2}\right\}$ must be averaged over the easy axes for $\eta$. If all directions in space were equally likely, then clearly $(\hat{\tau} \hat{\eta})^{2}$ would average to (1/3). The same result is also correct for cubic symmetry.

Diffuse magnetic scattering is observed with a mixed magnetic system. If one component of a birlary system occurs with a small concentration then the diffuse crosssection can be shown to be proportional to the square of the spatial Fourier transform of the magnetization defect created in the host matrix. Analysis of measurements on 
such systems has been central to the development of the theory of the electronic structure of magnetic alloys. As the temperature of the alloy is raised toward the critical temperature, the spatial range of the defect increases. In consequence, the diffuse cross-section as a function of $Q$ becomes increasingly narrow, and it is believed to be singular at the critical temperature.

Next we discuss the elementary properties of neutron-electron spectroscopy. To be concrete consider the matrix elements for transistions between J-multiplets in the energy-level diagram of a rare earth ion. The ion can be regarded as isolated, to a good approximation, and not subject to a significant molecular field, for example. The expression (17) is no longer valid since it is based on the relation $L+2 S=g \mathrm{~J}$ which is restricted to a J-multiplet.

The matrix element $<J M|L+2 S| J^{\prime} M^{\prime}>$ vanishes except for $\left|J-J^{\prime}\right|=1$; thus for small $\mathbf{Q}$ we observe dipole-allowed transitions [10]. Beyond the limit of small $\mathbf{Q}$ higher-order transitions contribute to the cross-section. Even though the higher-order transitions are very weak compared to dipole-allowed contributions they have been unambiguously observed [12]. It is found that the energy separation between multiplets is essentially the same in concentrated and dilute magnetic systems if the levels come from a single Coulomb term. Significant differences are seen in data for the two types of system for levels that belong to different terms. This feature is attributed to screening of the Coulomb interaction by conduction electrons.

Data on dilute systems is readily obtained by optical spectroscopy. This technique is constrained by the dipole-selection rule, and it is not useful for concentrated metallic magnets. At present, neutron-electron spectroscopy, which is free of both constraints, has been successfully applied to transitions with energy separations up to $1.8 \mathrm{eV}$, and the technique is very much in its infancy.

Another form of magnetic spectroscopy is the study of crystal field levels. This is now very well established as a tool for direct observation of the crystal field energy level scheme [13], in 3d, rare earth and actinide compounds.

The introduction of exchange interactions between ions couples the single-ion crystal 
field states discussed in the preceding paragraph. Collective excitations are formed from phase-related linear combinations of the single-ion transitions. These exciton states, as they are usually called, display significant dispersion which can be followed throughout the Brillouin zone in many cases. The limiting factor is energy, but this has been ameliorated with the development of advanced pulsed sources and appropriate time-of-flight instrumentation.

A spin wave is the coherent propagation of a single unit of spin deviation. It is in some senses the magnetic analogue of a phonon, in as much that the neutron cross-section vanishes unless there is simultaneous conservation of energy and wave vector. Spin wave and phonon excitations can usually be distinguished in the scattered spectrum by one of several simple tests. First, spin wave intensities decrease with increasing $Q$ because of the atomic form factor, whereas phonon intensities increase, with a $Q^{2}$ dependence. Secondly, a spin wave is an excitation away from an ordered state so it vanishes above the critical temperature. Hence, spin wave excitations are usually more sensitive than phonons to variations in temperature. On approaching the transition temperature spin waves soften and become more heavily damped.

Neutron polarization analysis affords a completely unambiguous method by which to identify spin waves [7] because creation or annihilation of a spin wave induces polarization in an unpolarized neutron beam. Phonons do not produce such an effect, as might be expected from physical intuition.

Polarization analysis is even more useful when it comes to isolatirng paramagnetic spin fluctuation scattering, particularly when it is a case of studying a material with a high transition temperature and hence a strong phonon background. In the case of a paramagnet, which does not possess a preferred axis, there is no creation of polarization. An initially polarized beam has a final polarization in the direction of $\mathbf{Q}$, and a magnitude controlled by the projection of the incident polarization onto $\mathbf{Q}$. Hence, the final polarization vanishes if the incident polarization and $\mathbf{Q}$ are perperidicular, and it achieves a maximum value when they are parallel.

The main problem in practice has been to obtain efficient methods of producing polarized beams or of analysing the polarization of a beam scattered by the sample. 
Inelastic scattering events are typically $10^{-3}$ of the elastic intensity, so a relatively inefficient polarizing method that is tolerable for elastic studies may render inelastic measurements impossible.

At the time of writing there is renewed interest in the dynamics of paramagnetic spin fluctuations. Various experimental groups have reported, over the past few years, careful measurements of the paramagnetic response of insulating (EuO, EuS) and metallic ( $\left.\mathrm{Fe}, \mathrm{Ni}, \mathrm{Pd}_{2} \mathrm{MnSn}\right)$ systems. The consensus opinion is that the response evolves with increasing $Q$ from a Lorentzian-like function centred at $\omega=0$ to a squarer or top-hat function. But recent data for Gd [14] shows a distinct peak at finite $\omega$ for large $Q$, near the zone boundary, with a strong dispersion. This feature persists deep into the paramagnetic region, and there is, of now, no satisfactory explanation of this sloppyspin-wave-like excitation. 


\section{Illustrative Examples}

The use of neutron scattering in condensed matter and materials research began in real earnest in the early part of the nirieteen fifties with particularly significant contributions coming from the USA and Cariada. Instrumentational concepts from this time survived although now vastly more efficient because of fast dedicated computers and the benefits of a steady evolution in the efficiency of collimators, monochromators and detectors.

Luminaries in the subject gathered in Stockholm in October 1957 for a meeting on the use of slow neutrons to investigate the solid state: the organizers, Pauli and Stedman, attracted Brockhouse, Egelstaff, de Gennes, Larsson, Palevsky, Riste and Waller, among others, as key speakers. Several experiments on solids and liquids, including the first observation of the roton minimum in the superfluid $\mathrm{He}^{4}$ phonon dispersion curve, were made at the R1 reactor in Stockholm (see fig. 1). New possibilities came with the commissioning of the R2 reactor at Studsvik in 1962, and routine operation of state of the art diffractometers and spectrometers. Flux at the sample on a Studsvik instrument was significantly higher than on a comparable instrument placed at the heavy water reactor R1 in Stockholm. Ph.D. theses submitted to the universities of Gothenburg, Stockholm and Uppsala in the period 1968-1974 by, amongst others, Nelin, Nilsson, Sköld and Stedman contained results which attracted much acclaim. Stedrnan's contributions both in original instrument design (double crystal monochromator and constant-q method in phonon spectroscopy) and very precise lattice dynamics studies, mapping Fermi surface features observed in Kohn anomalies, stand out. In fig. 2 the experimental achievement during this period at Studsvik is demonstrated. Significant theoretical work was published somewhat ahead of the experimental programme by Sjölander; his review article published in 1965 [19] stimulated research on liquids and solids. In the dawn of Sweden's neutron research programme scientific interests did not include magnetic phenomena and this situation largely prevails today.

The following subsections are written with the aim of imparting to the reader a flavour of scientific progress in a range of topics that have benefited frorn use of the neutron scattering technique. At the same time, we give a perspective on work by Swedish scientists, using instrumentation based at the Studsvik reactor arıd overseas facilities. 
An effort has been made to reference recent papers and review articles.

Unfortunately, any choice of topics runs the risk of being invidious. To those researchers who do not find their work cited we hope no lasting offence is taken because surely none is intended. There is no special order in which topics are presented. With such a wealth of material to draw from we recognize that other authors could select a quite different set of topics and papers and still fulfil the same aims.

\subsection{Collective excitations in quantum fluids}

Helium-3 has such a high absorption for low energy neutrons that it is a favoured material for detectors. On the other hand, it forms at low temperatures an almost perfect quantum Fermi liquid which is of interest in its own right and as a guide to other, more complicated, Fermi systems. Among these are electrons in metals, where there is the added complication of scattering by ion cores, nuclei which are finite size twocomponent systems, and neutron stars whose remoteness make them less than ideal subjects for microscopic studies.

One intriguing phenomenon in liquid $\mathrm{He}^{3}$ is the existence of a collective density oscillation which, in some respects, resembles a sound mode. However, as made clear by Landau in his seminal papers published in 1956, zero-sound is a purely quantum phenomenon, associated with distortions in the Fermi surface, which occurs in the collisionless regime of dynamics. Ordinary sound is a purely classical phenomenon which occurs in the hydrodynamic (collision dominated) regime of many particle dynamics.

The dispersion of zero-sound, obtained with inelastic neutron scattering, was first reported by Sköld et al. [20] (fig. 3). These experimentalists surmounted the seemingly daunting problem due to absorption by using a clever design for the sample container and counting for very long times (at a medium flux reactor) with a correlation chopper spectrometer. Since then the damping and pressure dependence of zero-sound has been measured [21]. Mixtures of the quantum liquids $\mathrm{He}^{3}$ and $\mathrm{He}^{4}$ (Fermi and Bose liquids, respectively) pose challenges to theoretical physicists. While existing theories 
are moderately successful when confronted with data [22] a number of interesting features remain unexplained.

\subsection{Are classical liquids hard sphere assemblies?}

By and large, the structure of a monatomic liquid is the same as that of an assembly of hard spheres of the appropriate radius and packing density. This is the conclusion of $x$-ray and neutron diffraction measurements of the static structure factor $S(Q)$ defined in eqn (11). Neutron diffraction data for the structure factor of liquid lead are showr in fig. 4 together with the appropriate hard-sphere values. One carı thus corıclude that the physically essential information obtainable from a $S(Q)$ is to be found in the relatively small difference between the measured curve and the corresponding hard-sphere quantity.

This is a sad state of affairs, since it means that it is a struggle to extract information on the molecular potential from measurements of $S(Q)$. It also means that measurements and data analysis must be performed with great attention to detail if useful results are to be obtained. Both have been refined to a state where molecular potentials are extracted through an iterative process in which computer simulation and neutron scattering results are compared.

Computer simulations and neutron scattering experiments go hand-in-hand. There is probably no better example of the impact of the twin approach than in the advancements made in the past few years in understanding the static and dynamic properties of liquids [23]. It is fair to say that up to quite recently simulation studies set a standard for neutron measurements, but now groups in Sweden (Dahlborg and collaborators) and the Netherlands (de Graaf and collaborators) have brought the accuracy of neutron measurements on a par with highest quality simulation data. It is even possible to go as far as obtaining from data correlation functions in space and time which are the natural co-ordinates in a computer simulation $[24,25]$.

Results for bismuth, a semi-metal, illustrate what can be achieved. First, a molecular potential has been constructed which yields simulation data in accord with measured 
values of $S(Q)$ (see fig. 5), including a shoulder on the large $Q$ side of the main peak in the structure factor that seems to be a characteristic feature of semi-metals [26] (and not present in $S(Q)$ for a hard-sphere assembly shown in fig. 4). Secondly, the selfparticle dynamics has been extracted from the data, even though bismuth is a totally coherent scatterer [25]. The data have then been analysed in terms of the mean-square displacement which, as expected from simple physical considerations, increases linearly with time at sufficiently long times with a gradient proportional to the diffusion constant. Values of the latter obtained from computer simulations and scattering experiments agree well with the observed value (fig.6). This is the first time this has been demonstrated. At intermediate times, before the diffusive region is reached, there is a significant discrepancy between simulation and neutron scattering results, which awaits a satisfactory explanation.

\subsection{Structure and dynamics in hydrogenous substances.}

Water, ever present in living matter, and other hydrogen bonded systems have been extensively investigated with $x$-ray and neutron scattering by several groups at almost all universities in Sweden. The amount of effort devoted to such systems reflects both their basic importance in chemistry and biology, and the very convenient neutron scattering properties of the proton and deuteron which allows use of the isotope substitutional technique. In this report we will not consider liquid water (although it has been studied extensively during the 60 's) and we refer the interested reader to a review article [27].

Protons are strong incoherent neutron scatterers, so the signal from a hydrogenous material is dominated by the properties of individual protons, whereas deuterons are predominantly coherent scatterers. Bragg scattering from deuterated crystals thus yields useful structural information whereas $X$-ray scattering is poor in locating protons because of the weak scattering power of hydrogen.

In conjunction with vibrational and NMR spectroscopy and theoretical calculations neutron and $x$-ray diffraction have been key tools in a long-standing hydrogen bond project co-ordinated by Olovsson at Uppsala University. This project has focussed on a 
broad range of structural and dynamical molecular-level aspects of the chemistry and physics of $\mathrm{H}$-bonded crystals. Neutron diffraction became early an essential technique in many of the problem areas studied by this group, such as structure and dynamics of water in solids [28], electron density mappings [29], the hydrated proton (ref. [30] and references therein), ferroelectricity and other physical properties [31], H-bond geometry and models for $\mathrm{H}$-bond interactions [32], order-disorder problems in $\mathrm{H}$-bonds [33] and proton conductors [34].

A recent example of work in this project is the determination of the electron density in $\mathrm{NaHC}_{2} \mathrm{O}_{4} \cdot \mathrm{H}_{2} \mathrm{O}$ at $120 \mathrm{~K}$, using $x$-ray and neutron diffraction techniques [35]. This is one of a limited number of investigations at low temperatures of the effect of the crystalline field on the charge density of the water molecule (see fig. 7). Deformation densities in the three independent hydrogen bonds are consistent with an electrostatic model for bonds of weak to intermediate strength in which polarization and other contributions become more important as the proton-acceptor distance decreases. The hydrogen bonding affects both the covalent bonds and the lone-pair electron density of the acceptor oxygen atorris.

Another example of the function of $\mathrm{H}$-atoms in crystals is the fact that the presence of hydrogen can induce structural ordering, although the mechanism for this is not always well understood. The $\mathrm{Pd}_{3} \mathrm{Mn}$ structure for example crystallizes in a disordered fcc structure above approximately $800 \mathrm{~K}$, and sluggishly transforms to an ordered tetragonal structure at lower temperatures. In the presence of hydrogen the initially disordered sample transforms to an ordered structure. As a first step toward understanding the mechanism of this type of ordering, the locations of the hydrogen atoms were determined by powder diffraction using a Rietveld profile refinement technique [36]. It was found that the deuterium atoms mainly occupy octahedral sites with six nearest-neighbour Pd sites (fig. 8).

Today powder diffraction methods in conjunction with the successful implementation of the Rietveld refinement technique scheme [37] has become a very useful structuresolving tool to the extent that it competes favourably with single-crystal work. 
6.4 Proton jumps and vibrations in hydrogen-metal systems.

Research on hydrogen-metal systems forms a small industry of work by chemists, physicists and metallurgists pursuing strategic and basic goals. Strategic work is aimed at energy storage, fusion technology, hydrogen embrittlement and the like. In addition, due largely to the small mass of the hydrogen, there are a variety of subtle effects such as fast hydrogen diffusion, large isotope effects, and hydrogen turnelling. Moreover, there is a conceptual relation between hydrogen and muon behaviour in metals [38].

At low concentrations, hydrogen atoms undergo a diffusive motion when viewed over long (macroscopic) times. The standard model assumes that diffusion occurs over welllocalized interstitial sites interconnected by a set of jump vectors e. The jump probability to any of the $n$ adjacent sites is $(1 / n \tau)$ where $\tau$ is the residence time at a given site. For a simple Bravais interstitial lattice the cross-section is a Lorentzian like eqn (14) with a half-width at half-height,

$\Gamma(Q)=(1 / n \tau) \sum_{j=1}^{n}\left[1-\cos \left(Q \cdot e_{j}\right)\right]\left\{=Q^{2} \ell^{2} / \tau ; Q \rightarrow 0\right\}$

where the second equality defines a length $\ell$ introduced in section 5.1.3.

The first experiment in which the quasielastic scattering from a metal hydride was interpreted in terms of diffusion was that by Sköld and Nelin [39] on hydrogen in fcc palladium ( $\alpha$-phase) in 1967 (see fig. 9). Bcc metals are more complicated to model. At room temperature the standard model works quite well, but at higher temperatures the measured width is significantly smaller than calculated values. Satisfactory agreement between measurements and theory is achieved when the latter is generalized to include a double-jump mechanism with sequences of two rapid jumps to nearest and next-nearest neighbor interstitial positions and also backward jumps.

Additional information can be extracted from measurements by analysing the intensity of the quasielastic line. In this respect, eqn (14) is modified by inclusion of a DebyeWaller factor $\left.\exp \left(-Q^{2}<u^{2}\right\rangle\right)$ where $\left\langle u^{2}\right\rangle$ is interpreted as the mean-square vibration amplitude of the hydrogen during its residence time. Values of $\left\langle u^{2}\right\rangle$ vary between 0.02-0.08 $\AA^{2}$ depending on the system and temperature. The value for hydrogen in palladium is particularly large, and this is attributed to the relatively low frequency of the 
localized mode in this system, mentioned already in section 5.1.3.

When viewed over short times a hydrogen atom vibrates about an interstitial site. This can be viewed as motion of a particle in a harmonic potential, centred at the interstitial site, to a good approximation. Better still, it is modelled by an impurity embedded in a matrix of atoms, as mentioned in section 5.1.3. The latter approach stresses the picture of a hydrogen atom as a probe of lattice dynamics, in much the same way as is customary when interpreting muon behaviour in a metal. Experiments on localized modes are very much improved by using a spallation source because they occur at energies around $130 \mathrm{meV}$ at which the intensity and resolution available at reactor based spectrometers has seriously degraded $[40,41]$.

\subsection{Zero-moment magnetic materials}

Magnets that contain rare earth or actinide ions (4f and $5 f$ unpaired electrons, respectively) possess a rich variety of features which can border on exotic. Rare earth magnets are relatively easy to understand because $4 f$ electrons are compact and hence not too strongly perturbed by the local environment and conduction electrons. Nonetheless, some properties, of even elemental rare earth magnets such as gadolinium, defy explanation with current theoretical ideas and methods [14,42].

But here we want to draw attention to an intriguing effect in some actinide magnets, namely, the almost complete cancellation of spin and orbital contributions which results in a zero total moment. Johansson and colleagues [43] attribute this to strong interactions between the $f$ and conduction electrons ( $s, p$ and $d$ ) aiding the interatomic correlations which produce oppositely aligned spin and orbital moments in the first half of $f$ electron series. They predict that $\mathrm{UFe}_{2}$ is an intinerant ferromagnet with a magnetic moment due mairly to the iron moments. From the discussion given in section 5.2 we conclude that the magnetic neutron scattering amplitude for the uranium ion should be very srnall near the forward scattering direction.

Wulff et al. [9] have confirmed this to be the case. The measured total uranium moment is zero within experimental error. The Q-dependence of the corresponding magnetic 
amplitude is very strong and in accord with theoretical predictions by Brooks et al [43].

\subsection{Arresting Relaxation in Liquids}

Our understanding of glasses is very much in its infancy. With silica glass, for example, it is commonly believed that the structure should contain $\mathrm{SiO}_{4}$ tetrahedra similar to those of the different forms of crystalline $\mathrm{SiO}_{2}$ [44]. The nature of short and medium range order is a subject of much current debate. None of the existing models is in complete accord with $\mathrm{x}$-ray and neutron diffraction work. Nor is there satisfactory agreement with available computer simulations. As with studies of liquids, there is a need for accurate data for the static structure factor $S(Q)$ and dynamic scattering function $S(Q, \omega)$ over wide ranges of $Q$ and $\omega$.

A new kind of intermediate range ordering has recently been observed in superionic borate, phosphate and molybdate glasses. It manifests itself in an anomalous peak in $S(Q)$ at a low $Q$ of $0.8 \AA^{-1}$ [45] (fig. 10). Torell and collaborators attribute this to the formation and ordering of Agl-clusters. There are strong indications from neutron diffraction in combination with a variety of other experimental results that the Agl clusters constitute a fractal percolating network within the host glass which serves as a conducting pathway for the $\mathrm{Ag}^{+}$ions $[46,47]$.

A theory of the glass transition developed by Sjölander and collaborators [48-52] is based on the premise that the transition is basically of a dynamic nature, which stems from changes in the self-diffusion constant and viscosity, rather than driven by a mode softening at the homogeneous nucleation limit. In a supercooled liquid structural relaxation times, governed by atomic diffusion, are very long. Ultimately, a glass state is formed in which atoms form a disordered solid, structural degrees of freedom are frozen out, and structural relaxation times are truly macroscopic. At an atomic level, motion of a single tagged particle involves a non-linear coupling of self- and collective motions which gives rise to a cage effect where the medium blocks the particle's passage. The cage effect progresses to self-trapping in the super-cooled state, due to the slow response of the liquid to disturbances, and this results in a small value of the self-diffusion constant. The theory focuses on the normalized intermediate scattering function which is postulated to have a particular functional shape. 
These concepts have largely been vindicated by computer simulation [53-55] and neutron scattering studies [56-58]. In particular, there is good evidence for the predicted two slow-relaxation steps, i.e. slow on the time scale of normal lattice vibrations (see fig. 11). One of the two steps exists on both sides of the transition temperature and slows down critically. The second step exists only in the liquid phase, and when the transition is approached the divergence of the associated time scale outstrips the time scale for the first step. There is also a divergence of transport constants, as might be expected. However, since the transition is not a continuous phase transition, as in a simple magnet, established universality classes and scaling concepts do not apply. Even so, theory predicts that the second relaxation process obeys a master curve, which is a form of scaling, and this feature is indeed borne out by experiment.

\section{Future trends}

The few examples discussed above just give a flavour of the overwhelming possibilities of the neutron scattering technique. Many areas were not even mentioned such as the small-angle scattering technique by which important contributions to many different kinds of sciences has been made. On the one hand, the neutron scattering technique complements other experimental methods, like $\mathrm{X}$-ray and light scattering, but in many instances it provides information not available by other means. It has also the advantage of being a direct probe which makes meaningful comparisons between theoretical predictions and experimental results feasible provided, of course, that the experimental data has been treated with up-to-date methods with respect to data correction procedures.

What are the general trends and how will the technique develop in the future?

Several different research areas of increased interest can be identified. To be mentioned here is especially the enhanced use of polarized neutrons which has been made possible through the development of efficient polarizers, above all the supermirror [7]. This has resulted in an efficient tool that makes it easy to distinguish between nuclear and magnetic phenomena in a scattering experiment and this can now be used with advantage also at reactors of medium source strength. The construction of a facility for 
polarized neutrons at the $\mathrm{R} 2$ reactor is in line with this development and it is anticipated that it will be of great use for Swedish scientists in the future. Other developments which also certainly will continue is the increased interest in studies of complex chemical systems, amorphous materials (both magnetic and non-magnetic), thin films, biological substances, the energy levels of the crystal field in solids, etc.

Another insight which has slowly emanated over the years among neutron scattering scientists is the observation that one experimental technique alone is not able to give answers to all questions. This does also apply to the neutron scattering technique and thus a stronger and stronger interplay between neutron scattering and other experimental methods (X-ray diffraction, light scattering and absorption, molecular dynamics simulations) have to be employed in order to map static and dynamic phenomena especially in complex systems. Complex in this respect means both systems which behave in a complicated and subtle way, but also chemically complex. As an example we can mention ordinary water, the behaviour and interaction of which on a molecular level, even after intense scientific onslaught over many decades, is to a large degree unexplained, materials of technological interest like soft amorphous and partly crystalized magnetic materials.

In order to extract some relevant information today from a diffraction or from a neutron inelastic scattering experiment it is necessary not only to have high quality data (good statistics, large enough $Q-\omega$ range, appropriate resolution in order to unravel the desired information from the measured data, etc) but also to have as a full control of the experiment itself as possible. What is even more important is that the experimentalist must be able to accurately correct the data for all the experimental effects. These criteria work hand in hand and to improve one and not the others to the same degree is meaningless. For example, with the advent of dedicated spectrometers for measurements of dynamic scattering functions $S(Q, \omega)$ at ILL, ISIS, and Studsvik it is thus essential to put a lot of effort into the development of accurate correction procedures. In order to optimize the experimental accuracy it will be more and more common to simulate the complete experiment on a computer by Monte-Carlo methods before it is actually performed.

It is definitely so that the pulsed neutron sources, especially ISIS, have brought a new dimension into the neutron scattering field and that they will be of outstanding 
importance (two examples only to be mentioned) for studies of magnetic phenomena and for studies of high-energy collective excitations at small Q. It should however be pointed out that a high-flux research reactor like the one at ILL and the ISIS spallation source are complementary. Both kinds of sources are needed in order to cover in the best possible way the vast research areas where the neutron scattering technique can give unique and substantial information about microscopic phenomena. It has also to be observed that this does not mean that the scientific case for medium flux research reactors, like $\mathrm{R} 2$, is defunct. There are many examples where the quality of the data obtained at for example R2 or other smaller research reactors are as good as those obtained at the IIL. We should not dwell into explaining the reasons for this but rather state it as a fact. It is certainly also so that the exploratory measurements in the future preferably are to be made at smaller centres as well as those experiments requiring very complicated and fancy auxiliary equipment. 
Acknowledgements

We are grateful to K. Hermansson, E.B. Karlsson, I. Olovsson, K. Sköld, R. Tellgren, J.O. Thomas and L.M. Torell for discussions and correspondence. 
References

1. Scherm, R.H. in The Art of Measurement, edited by B. Kramer (VCH, Weinheim 1988)

2. Lovesey S.W. Theory of Neutron Scattering from Condensed Matter, Vols 1 and 2 , 3rd impression (Oxford University Press, Oxford, 1987)

3. Neutron Scattering, Methods of Experimental Physics Vols 23A,B,C, edited by D.L. Price and K. Sköld (Academic Press, N.Y. 1986)

4. Neutron Scattering at a Pulsed Source, edited by R.J. Newport, B.D. Rainford and R. Cywinski (Adam Hilger, Bristol, 1988)

5. Lovesey, S. W., Inst. Phys. Conf. Ser. No 101, 133 (1990).

6. Moon, R.M. and West, C.D., Physica 137B, 347 (1986)

7. Williams, W.G., Polarized Neutrons (Oxford University Press, Oxford, 1988).

8. Bee, M., Quasi-elastic Neutron Scattering (Adam Hilger, 1989)

9. Williams, R.D. and Lovesey, S.W., Z. Phys. B62, 413 (1986)

10. Wulff, M., Lander, G. H., Lebech, B. and Delapalme, A., Phys Rev B39, 4719 (1989)

11. Williams, W.G., Boland, B.C., Bowden, Z.A., Taylor, A.D., Culverhouse, S. and Rainford, B.D., J. Phys. F17, L151 (1987)

12. Taylor, A.D., Osborn, R., McEwen, K.A., Stirling, W.G., Bowden, Z.A., Balcar, E. and Lovesey, S.W., Phys. Rev. Lett. 61, 1309 (1988). 
13. Fulde, P. and Loewenhaupt, M., Adv. Phys. 34, 589 (1986).

14. Cable, J.W., and Nicklow, R.M., Phys. Rev. B39, 11732 (1989).

15. Palevsky, H., Otnes, K. and Larsson, K.-E., Phys. Rev. 112, 11 (1958)

16. Brockhouse, B.N. and Stewart, A.T., Rev. Mod. Phys. 30, 236 (1958)

17. Larsson, K.-E., Dahlborg, U. and Holmryd, S., Ark. Fys. 17, 369 (1960)

18. Stedman, R. and Nilsson, G., Phys. Rev. 145, 492 (1966)

19. Sjölander, A., chap. 7 in Thermal Neutron Scattering, edited by P.A.Egelstaff, Academic Press, London and New York, 1965

20. Sköld, K., Pelizzari, C.A., Kleb, R. and Ostrowski, G., Phys. Rev. Letters 37, 842 (1976)

21. Scherm, R., Guckelsberger, K., Fak, B., Sköld, K., Dianoux, A. J., Godfrin, H. and Stirling, W.G., Phys. Rev. Lett. 59, 217 (1987).

22. Scherm, R., Dianoux, A.J., Fak, B., Guckelsberger, K., Körfer, M and Stirling, W.G., Physica B 156 and 157, 311 (1989).

23. Larsson, K-E., Phys. Chem. Liq. 12, 273 (1983)

24. Dahlborg, U., Söderström, O., Gudowski, W., Larsson, K.-E. and Davidovic, M., J .Phys. F: Met. Phys. 15, 2053 (1985)

25. Dahlborg U, Gudowski, W. and Davidovic, M., J. Condens. Matter 1, 6173 (1989).

26. Dzugutov, M. and Dahlborg, U., Phys. Rev. A40, 4103 (1989).

27. Hermansson, K., Portugaliae Physica 19, 133 (1988). 
28. Eriksson, A. and Hermansson, K., Acta Cryst. B 39, 703 (1983)

29. Hermansson, K., Acta Cryst. B 41, 161 (1985)

30. Gustafsson, T., Acta Cryst. C 43, 816 (1987)

31. Liminga, R. and Tellgren, R., Acta cryst. B 38, 1551 (1982)

32. Olovsson, I. and Jönsson, P.G., chap. 8 in The hydrogen bond - recent developments in theory and experiments, edited by P.Schuster et al. (North-Holland Publ. Co, Amsterdam) 1976.

33. Thomas, J.O., Tellgren, R. and Olovsson, I., Acta Cryst. B 30, 2540 (1974)

34. Thomas, J.O. and Farrington, G.C., Acta Cryst B 29, 227 (1983)

35. Delaplane, R.G., Tellgren, R. and Olovsson, I., Acta Cryst. B 46, 361 (1990)

36. Ahlzen, P.-J., Andersson, Y., Tellgren, R., Rodic, D., Flanagan, T.B. and Sakamoto, Y.Z., Z. Phys. Chem. 163, 213 (1989)

37. Rietveld, H.M., J. Appl. Phys. 2, 65 (1969)

38. Karlsson, E.B. Int. J. Quan. Chem. XXXV, 779 (1989)

39. Sköld, K. and Nelin, G., J. Phys. Chem. Solids 28, 2369 (1967)

40. Bennington, S.M., Benham, M.J., Ross, D.K., Taylor, A.D. and Bowden, Z.A., Z. Phys. Chem. 164, 1071 (1989)

41. Noreus, D. and Tomkinson, J., Chem. Phys. Lett. 154, 439 (1989) 
42. Hartmann, O., Karlsson, E.B., Wäppling, R., Chappert, J., Yaouanc, A., Asch, L., and Kalvius, G.M., J. Phys. F: Met Phys. 16, 1593 (1986).

43. Brooks, M.S.S., Eriksson, O., Johansson, B., Franse, J.J.M., and Frings, P.H., J. Phys F 18, L33 (1988).

44. Graneli, B. and Dahlborg, U., J. Non-Cryst. Solids 109, 295 (1989).

45. Börjesson, L., Torell, L.M., Dahlborg, U., and Howells, W.S., Phys. Rev. B 39, 3404 (1989)

46. Börjesson, L., Torell, L.M. and Howells, W.S., Phil. Mag. B 59, 105 (1989)

47. Börjesson, L. and Howells, W.S., Solid State lonics (in press).

48. Bengtzelius, U., Götze, W. and Sjölander, A., J. Phys. C 17, 5915 (1984)

49. Bengtzelius, U. and Sjögren, L., J. Chem. Phys. 85, 1744 (1986)

50. Götze, W., Z. Phys. B 60, 195 (1984)

51. Götze, W., Phys. Scripta 34, 66 (1986)

52. Götze, W. and Sjögren, L., Z. Phys. B 65, 415 (1987)

53. Signorini, G.F., Barrat, J-L., and Klein, M.L., J. Chem. Phys 92, 1294 (1990)

54. Kinell, T. and Lovesey, S.W., J. Phys. C 19, L791 (1986)

55. Dzugutov, M. and Dahiborg, U., Proc. from International Discussion Meeting on Relaxations in Complex Systems, Heraklion June 18-29, 1990. (to be published)

56. Mezei, F., Knaak, W. and Farago, B., Eur. Phys. Lett. 7, 529 (1988) 
.066 I | | I t

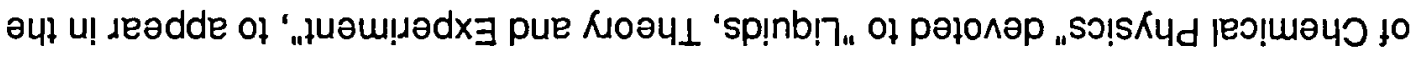

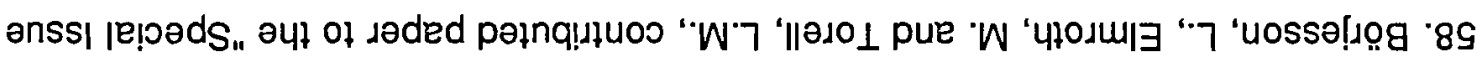

(686!

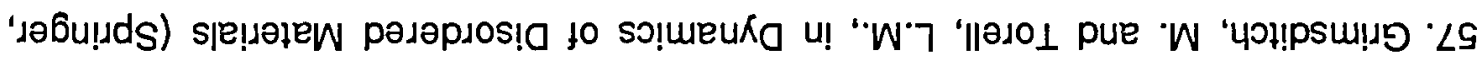
$8 \varepsilon$ 
Table 1.

Neutron scattering and absorption data for some selected elements (taken from a compilation by L.Koester and W.B.Yelon, 1982.)

\begin{tabular}{|c|c|c|c|c|c|}
\hline Element & $A$ & $\%$ abundance & $b$ & $\sigma_{\mathrm{s}}$ & $\sigma_{\mathrm{a}}$ \\
\hline \multirow[t]{3}{*}{$\mathrm{H}$} & 1 & 100. & -0.3741 & 81.87 & 0.3326 \\
\hline & 2 & 0.0149 & 0.6674 & 7.63 & 0.000519 \\
\hline & 3 & & 0.494 & 3.03 & $<0.000006$ \\
\hline \multirow[t]{3}{*}{$\mathrm{He}$} & $*$ & & 0.326 & 1.21 & $<0.001$ \\
\hline & 3 & 0.00014 & 0.574 & 5.6 & 5333. \\
\hline & 4 & 100. & 0.326 & 1.21 & $\sim 0$ \\
\hline \multirow[t]{4}{*}{$\mathrm{Li}$} & * & & -0.203 & 1.40 & 70.5 \\
\hline & 6 & 7.5 & 0.187 & 0.98 & 940. \\
\hline & & & $-0.026 i$ & & \\
\hline & 7 & 92.5 & -0.220 & 1.44 & 0.0454 \\
\hline \multirow[t]{4}{*}{$c$} & * & & 0.6648 & 5.564 & 0.00350 \\
\hline & 12 & 98.89 & 0.6653 & 5.564 & 0.00353 \\
\hline & 13 & 1.11 & 0.62 & 5.5 & 0.00137 \\
\hline & 14 & & & & $<10^{-6}$ \\
\hline \multirow[t]{4}{*}{0} & $\star$ & & 0.5805 & 4.234 & 0.00019 \\
\hline & 16 & 99.762 & 0.5805 & 4.234 & 0.00019 \\
\hline & 17 & 0.038 & 0.578 & 4.20 & 0.24 \\
\hline & 18 & 0.200 & 0.584 & 4.3 & 0.00016 \\
\hline $\mathrm{Na}$ & 23 & 100. & 0.363 & 3.23 & 0.530 \\
\hline \multirow[t]{3}{*}{$\mathrm{Cl}$} & * & & 0.9579 & 16.63 & 33.5 \\
\hline & 35 & 75.77 & 1.17 & 21.62 & 44.1 \\
\hline & 37 & 24.23 & 0.308 & 1.2 & 0.433 \\
\hline \multirow[t]{4}{*}{$\mathrm{K}$} & * & & 0.367 & 2.10 & 2.1 \\
\hline & 39 & 93.258 & 0.379 & 2.19 & 2.1 \\
\hline & 40 & 0.012 & & & 34. \\
\hline & 41 & 6.73 & 0.258 & 0.83 & 1.46 \\
\hline
\end{tabular}




\begin{tabular}{|c|c|c|c|c|c|}
\hline \multirow{3}{*}{ V } & * & & -0.0382 & 4.953 & 5.08 \\
\hline & 50 & 0.25 & & & 60. \\
\hline & 51 & 99.75 & -0.0414 & 4.946 & 4.9 \\
\hline \multirow{5}{*}{$\mathrm{Fe}$} & $\star$ & & 0.954 & 11.66 & 2.56 \\
\hline & 54 & 5.8 & 0.42 & 2.2 & 2.3 \\
\hline & 56 & 91.72 & 1.01 & 12.8 & 2.6 \\
\hline & 57 & 2.2 & 0.23 & $<1$ & 2.5 \\
\hline & 58 & 0.28 & 1.5 & 28. & 1.28 \\
\hline \multirow[t]{6}{*}{$\mathrm{Ni}$} & * & & 1.03 & 17.56 & 4.5 \\
\hline & 58 & 68.27 & 1.44 & 25.87 & 4.6 \\
\hline & 60 & 26.10 & 0.28 & 0.96 & 2.9 \\
\hline & 61 & 1.13 & 0.76 & 7.23 & 2.5 \\
\hline & 62 & 3.59 & -0.87 & 9.6 & 14.5 \\
\hline & 64 & 0.91 & -0.038 & 0.02 & 1.52 \\
\hline \multirow[t]{3}{*}{$\mathrm{Ag}$} & * & & 0.597 & 5.09 & 63.3 \\
\hline & 107 & 51.83 & 0.764 & & 38. \\
\hline & 109 & 48.17 & 0.419 & & 91. \\
\hline \multirow[t]{5}{*}{$\mathrm{Pb}$} & * & & 0.9401 & 11.11 & 0.17 \\
\hline & 204 & 1.4 & & & 0.66 \\
\hline & 206 & 24.1 & & & 0.0305 \\
\hline & 207 & 22.1 & & & 0.709 \\
\hline & 208 & 52.4 & & & 0.00049 \\
\hline $\mathrm{Bi}$ & 209 & 100. & 0.8533 & 9.156 & 0.033 \\
\hline
\end{tabular}

$b\left(10^{-12} \mathrm{~cm}\right)$ Coherent scattering length for bound atoms. Complex values correspond to a neutron wave length of $1 \AA$.

$\sigma_{\mathrm{s}}$ (barns) Total scattering cross sections of bound atoms for thermal neutrons

$\sigma_{a}$ (barns) Absorption cross sections for thermal neutrons ( $2200 \mathrm{~m} / \mathrm{sec}$ )

Natural isotope mixture 
Table 2.

Characteristic parameters of neutron-producing reactions used in neutron sources

$\begin{array}{lllll}\text { reaction } & \begin{array}{l}\text { number of } \\ \text { neutrons }\end{array} & \text { example } & \begin{array}{l}\text { neutron } \\ \text { production }\end{array} & \begin{array}{l}\text { target } \\ \text { power }\end{array} \\ \text { fission } & -2.5 \text { per } & \text { ILL high-flux } & 2 \times 10^{18} \mathrm{n} / \mathrm{s} & 57 \mathrm{MW} \\ & \text { fission } & \text { reactor } & & \\ & (1 \text { effective) } & \text { (continuous) } & & \end{array}$

$\begin{array}{lllll}\text { spallation } & -26 \text { per } 800 & R A L \text { spallation } & 3 \times 10^{16} \mathrm{n} / \mathrm{s} & \sim 0.2 \mathrm{MW} \\ \text { in } U^{238} & \text { MeV proton } & \text { source (pulsed) } & \end{array}$


Figure captions

Fig. 1. Dispersion curve for the excitations in superfluid ${ }^{4} \mathrm{He}$ at about $1.5 \mathrm{~K}$. The dashed line is the prediction of the Landau-Feynman theory. (from Palevsky et al [15])

Fig.2. Demonstration of the enormous increase in accuracy of the measured dispersion curves during the late 50 's and early 60 's. As example are results for aluminium in the [200] direction of the Brillouin zone taken. a) Brockhouse and Stewart 1958 [16], b) Larsson et al 1960 [17], c) Stedman and Nilsson 1966 [18]. The dashed and full lines correspond to different theories.

Fig. 3. a) Example fit of a phenomenological function to measured data. The dashed curves are the individual components of the fit (spin fluctuation, zero-sound and multi-pair) and the solid cirve is the sum. b) Dispersion relation for collective ("zero-sound") excitations in liquid ${ }^{3} \mathrm{He}$. Solid curves are theoretical predictions and dashed line an extrapolation of the zero-sound velocity at low frequencies. (Sköld, private communication)

Fig. 4. Measured $S(Q)$ for liquid lead (circles) and calculated curve according to the Percus-Yevick approximation for hard spheres.

Fig. 5. Comparison between the zeroth moments of the dynamic scattering function $S(Q)$ obtained from an optimized molecular dynamics simulation procedure [ ] and experimental results. The small $Q$ region is shown in the inset. (from Dzugutov and Dahiborg [26])

Fig. 6. The mean square displacement (MSD) of atom obtained from the width of the self correlation correlation function $G_{s}(r, t)$ for liquid bismuth. The full curve corresponds to molecular dynamics simulation results. (from Dahlborg et al [25]) 
Fig. 7. (a) The geometry of $\mathrm{NaHC}_{2} \mathrm{O}_{4}$ at $120 \mathrm{~K}$. Thermal ellipsoids are plotted at $75 \%$ probability. (b) to (d) show the experimental deformation densities in the $\mathrm{HC}_{2} \mathrm{O}_{4}$ ion. Each composite map has been plotted separately through the $O(1), C(1)$, $O(2)$ and $O(3), C(2), O(4)$ planes. (b) $X-N$ difference density, (c) high-order $X$ $(X, N)$ difference density, (d) dynamic $(X, N)$ multipole deformation density. Contours are at intervals of $0.05 \mathrm{e}^{-3}$. Solid and dashed lines denote positive and negative contours, respectively; the zero-level has been omitted. (from Delaplane et al. [35])

Fig.8. The tetragonal structure of $\mathrm{Pd}_{3} \mathrm{Mn}$. The coordinations of the two deuterium positions are shown in the lower part of the figure. In the upper part, the stacking sequence is indicated. (from Ahizen et al. [36])

Fig. 9. Observed width of the scattering function in $\mathrm{Pd}-\mathrm{H}$ for three temperatures. The curves predicted by the octahedral model are shown by the solid lines for the best value of $\tau$ at each temperature. Dashed line shows the curve predicted by the tetrahedral model for an arbitrary chosen value of $\tau$. (from Sköld and Nelin [39])

Fig. 10. The structure factors for two $(\mathrm{Agl})_{\mathrm{x}}-\left(\mathrm{Ag}_{2} \mathrm{O}-2 \mathrm{~B}_{2} \mathrm{O}_{3}\right)_{1-x}$ glasses with $\mathrm{x}=0.6$ (upper curves) and $x=0.1$ (lower curves) measured on the liquid and amorphous diffractometer LAD at ISIS at scattering angles (a) $146^{\circ}$ and (b) $10^{\circ}$. The upper curves have been shifted by 0.3 units to higher values for clarity. The difference between the intensities of the $x=0.5$ (upper curve), the $x=0.3$ (lower curve) glass and the undoped binary glass $x=0$ is shown in (c) for the $10^{\circ}$ scattering angle. (from Börjesson et al [46])

Fig.11. The normalized density correlation function at the reduced temperature 0.5 . solid line: $Q \sigma=7$; dashed line: $Q \sigma=6.2$; chain-dotted line: $Q \sigma=5.4 . \sigma$ is a characteristic length and $\tau$ is characteristic time related to the particular shape of the assumed interaction potential. The squares represent fits of Kohlrausch functions. The time scales of two different relaxation mechanisms are clearly seen. (from Dzugutov and Dahlborg [55]) 


\section{INSTRUMENTATION FOR THERMAL NEUTRON SCATTERING AT THE REACTOR R2, STUDSVIK}

\begin{tabular}{|c|c|c|}
\hline $\begin{array}{l}\text { Neutron } \\
\text { beam tube }\end{array}$ & Instrumentation & Responsible \\
\hline $\mathrm{H} 1$ & Service instrument & $\begin{array}{l}\text { NFL } \\
\text { (scientist: W.Gudowski) }\end{array}$ \\
\hline $\mathrm{H} 3$ & $\begin{array}{l}\text { Time-of-flight spectrometer } \\
\text { for thermal neutrons } \\
\text { (operational summer 1990) }\end{array}$ & $\begin{array}{l}\text { Department of Neutronphysics, } \\
\text { KTH, Stockholm } \\
\text { (scientist: U.Dahlborg) }\end{array}$ \\
\hline H5 & Triple-axis spectrometer & $\begin{array}{l}\text { Department of Neutron Research, } \\
\text { UU, Studsvik (scientist: K.Sköld) }\end{array}$ \\
\hline $\mathrm{H} 6$ & $\begin{array}{l}\text { Diffractometer for protein } \\
\text { studies } \\
\text { (operational winter 1990) }\end{array}$ & $\begin{array}{l}\text { NFL } \\
\text { (scientist: L.Sjölin, CTH) }\end{array}$ \\
\hline $\mathrm{H} 7$ & $\begin{array}{l}\text { Diffractometer for } \\
\text { disordered structures } \\
\text { (operational winter 1990) }\end{array}$ & $\begin{array}{l}\text { NFL } \\
\text { (scientist: R.Delaplane) }\end{array}$ \\
\hline H8 & Powder diffractometer & $\begin{array}{l}\text { Department of Chemistry, UU } \\
\text { (scientist: R.Tellgren) }\end{array}$ \\
\hline $\mathrm{H} 9$ & $\begin{array}{l}\text { Single-crystal diffractometer } \\
\text { (operational but temporarally } \\
\text { placed at beam tube } H 3 \text { ) }\end{array}$ & $\begin{array}{l}\text { Department of Chemistry, UU } \\
\text { (scientist: R.Tellgren) }\end{array}$ \\
\hline $\mathrm{H} 10$ & $\begin{array}{l}\text { Time-of-flight spectrometer } \\
\text { for cold neutrons } \\
\text { (operational winter 1991) }\end{array}$ & $\begin{array}{l}\text { Department of Neutronphysics, } \\
\text { KTH, and NFL } \\
\text { (scientists: U.Dahlborg and K.Sköid) }\end{array}$ \\
\hline $\mathrm{H} 10 \mathrm{~A}$ & $\begin{array}{l}\text { Spectrometer for polarized } \\
\text { neutrons } \\
\text { (operational winter 1991) }\end{array}$ & $\begin{array}{l}\text { Department of Neutronphysics, } \\
\text { KTH, and NFL } \\
\text { (scientist: U.Dahlborg) }\end{array}$ \\
\hline
\end{tabular}

KTH: Royal Institute of Technology, Stockholm; tel. 08/7906396

UU: Uppsala University; tel. 018/183776

CTH: Chalmers University, Gothenburg; tel. 031/722856

NFL: Neutron Research Laboratory, Studsvik; tel. $0155 / 21852$ (UD), 21843 (RD), 21849 (KS) 


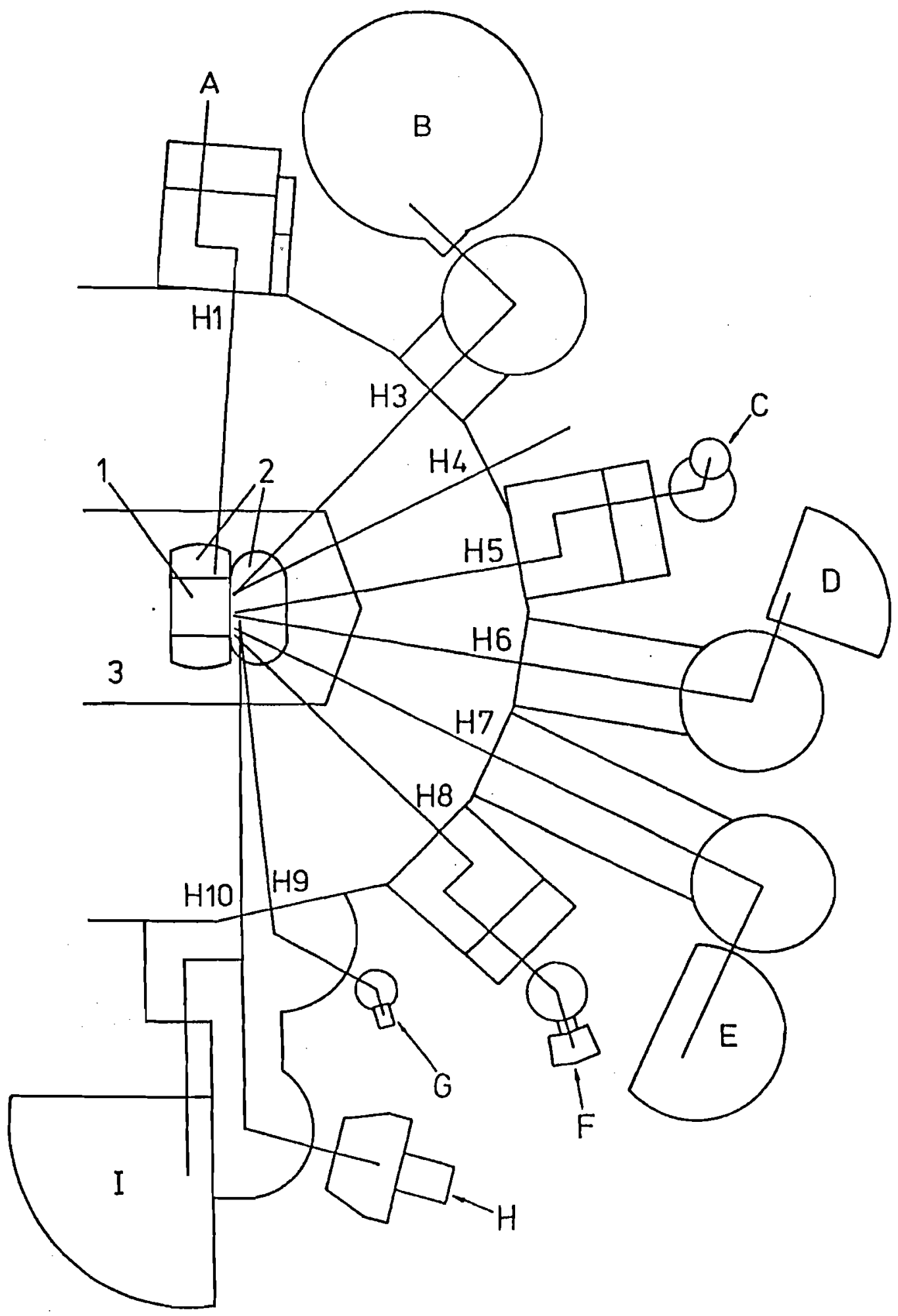

Diagram shows layout of the instrumentation around R2, Studsvik.

1) Reactor core (enriched ${ }^{235} \mathrm{U}$ and $\mathrm{H}_{2} \mathrm{O}$ ); 2) $\mathrm{D}_{2} \mathrm{O}$ moderator; 3) reactor pool 


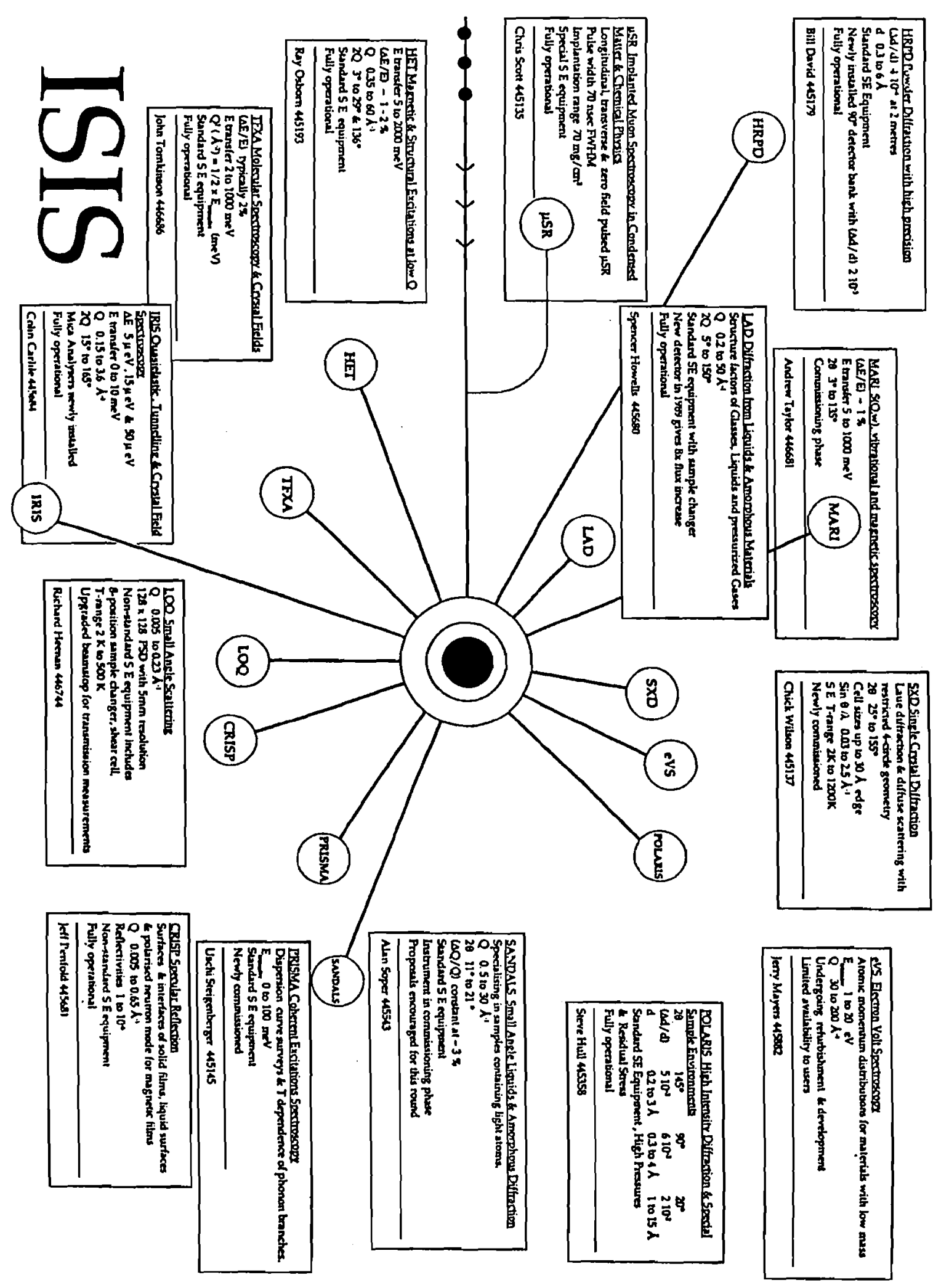

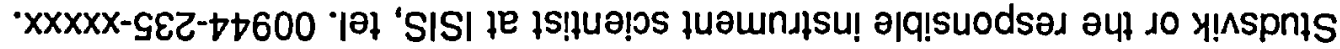

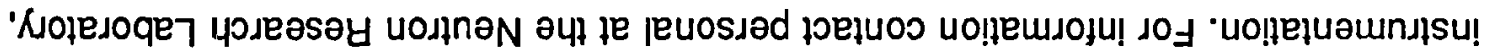

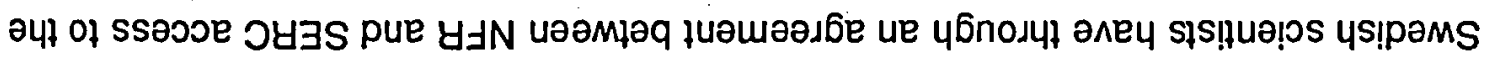




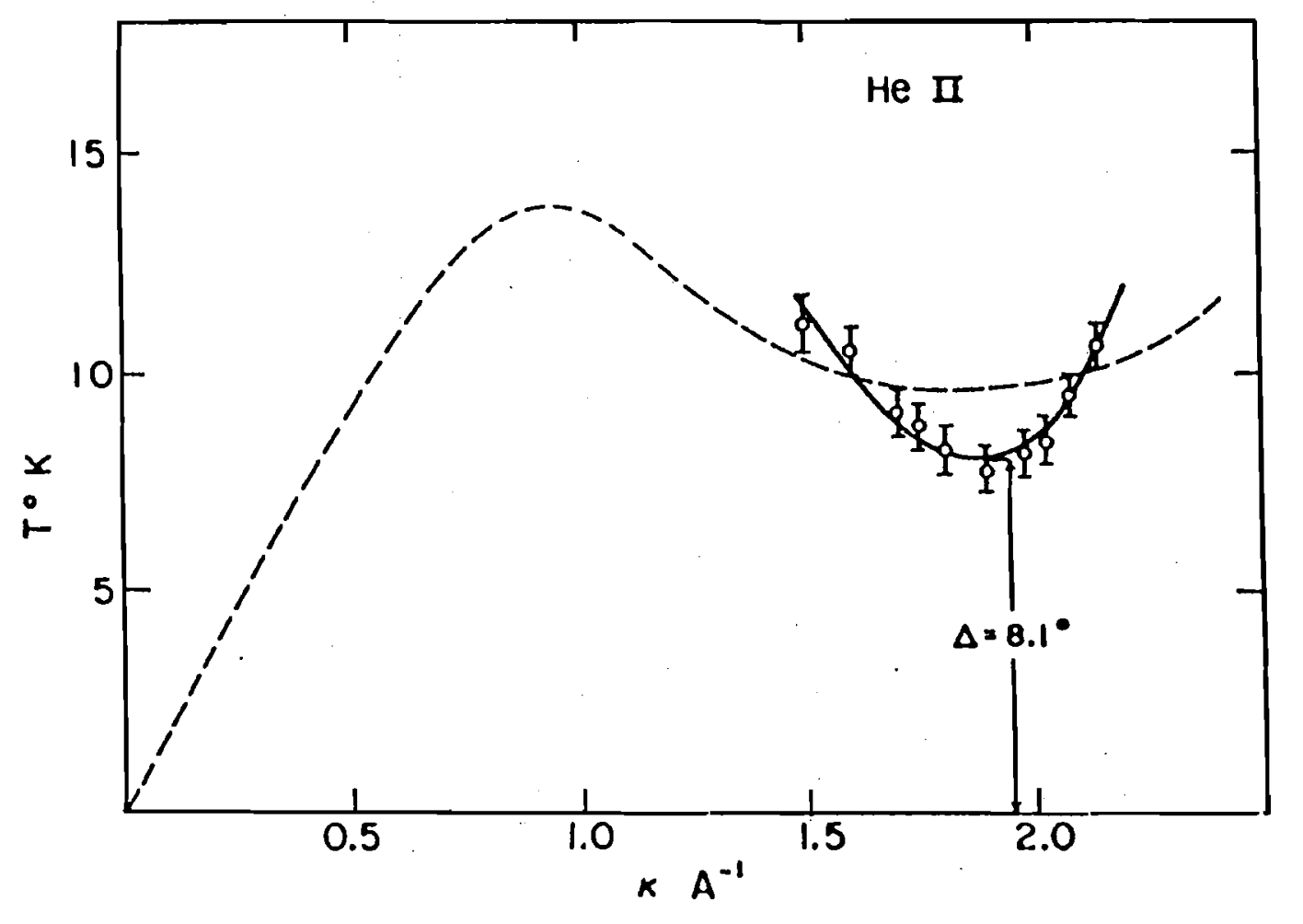

Fig. 1 
2. 占里
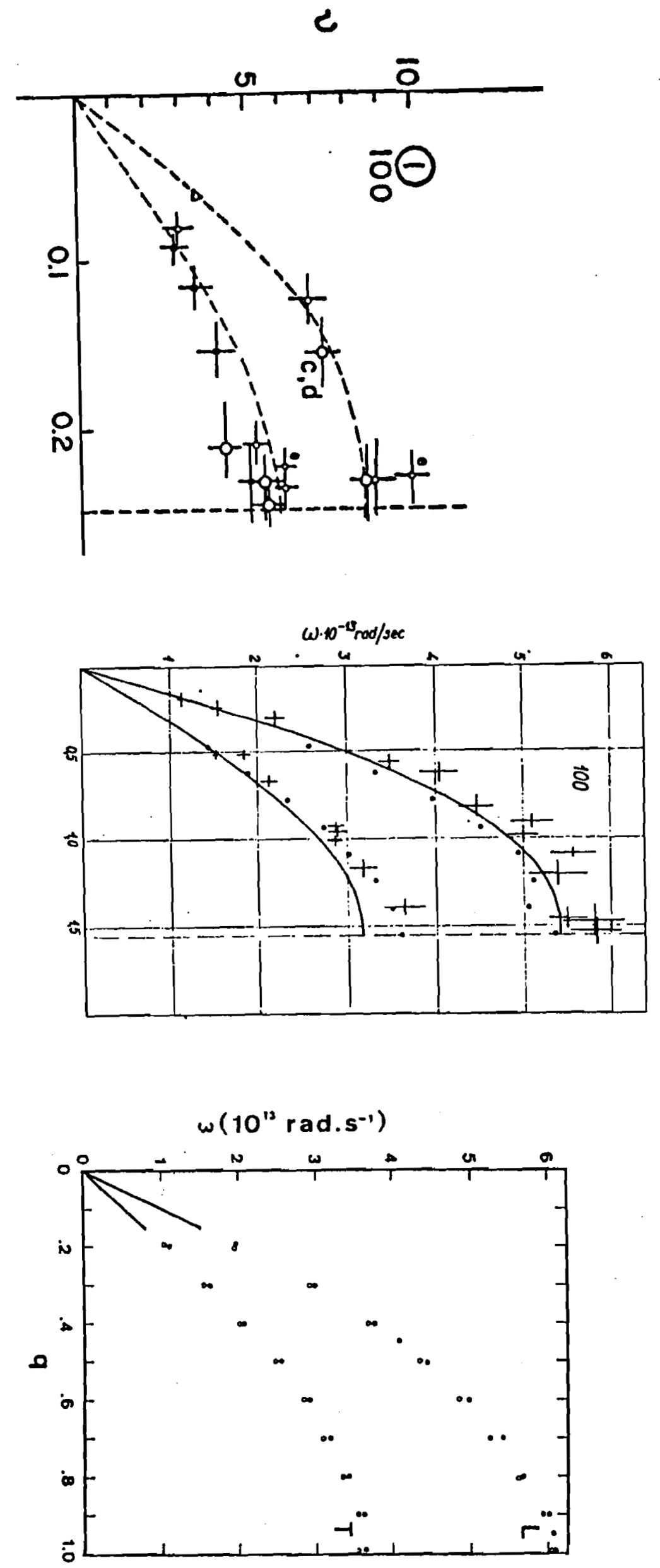

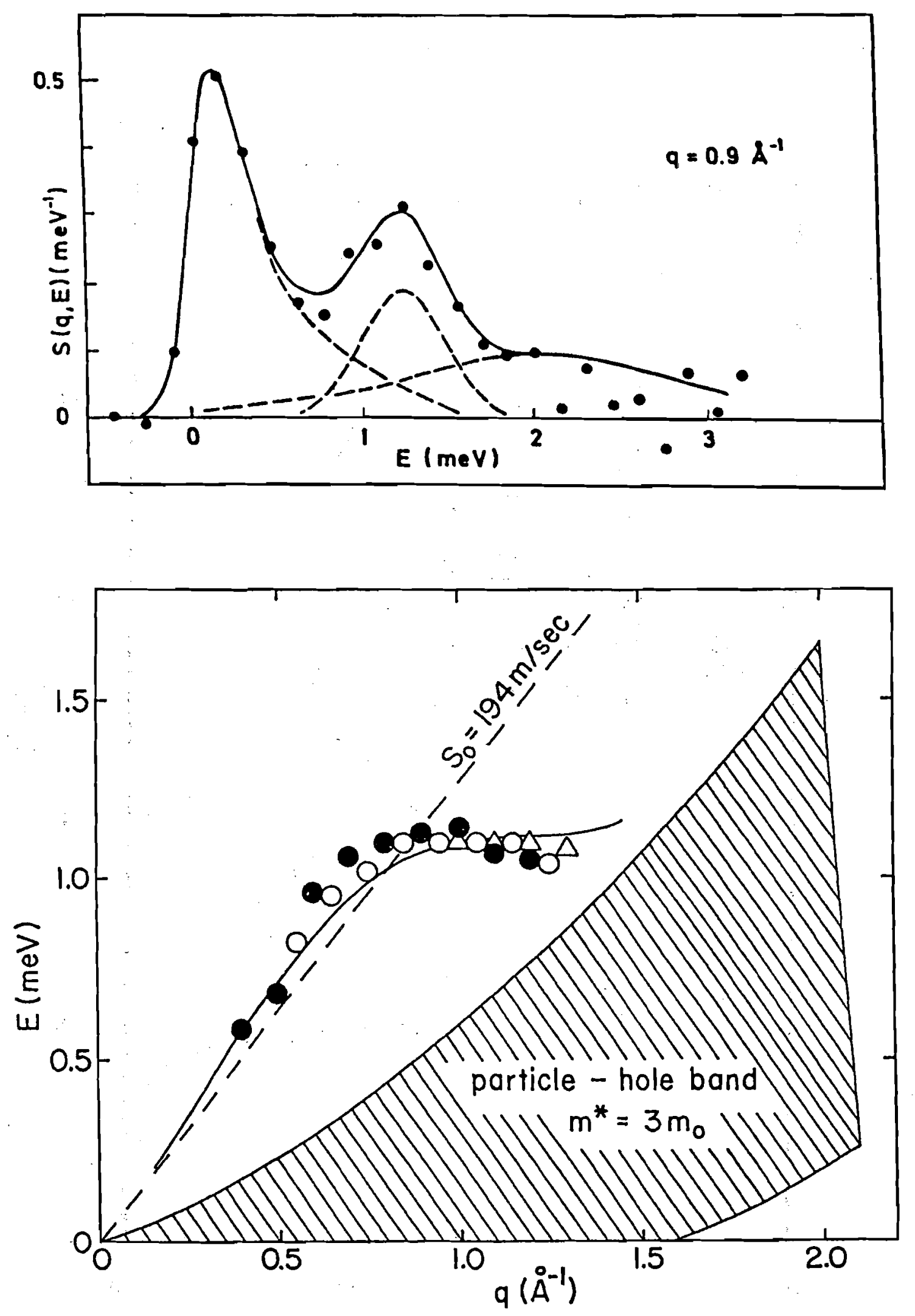

$$
\text { Figs }
$$


4 粲

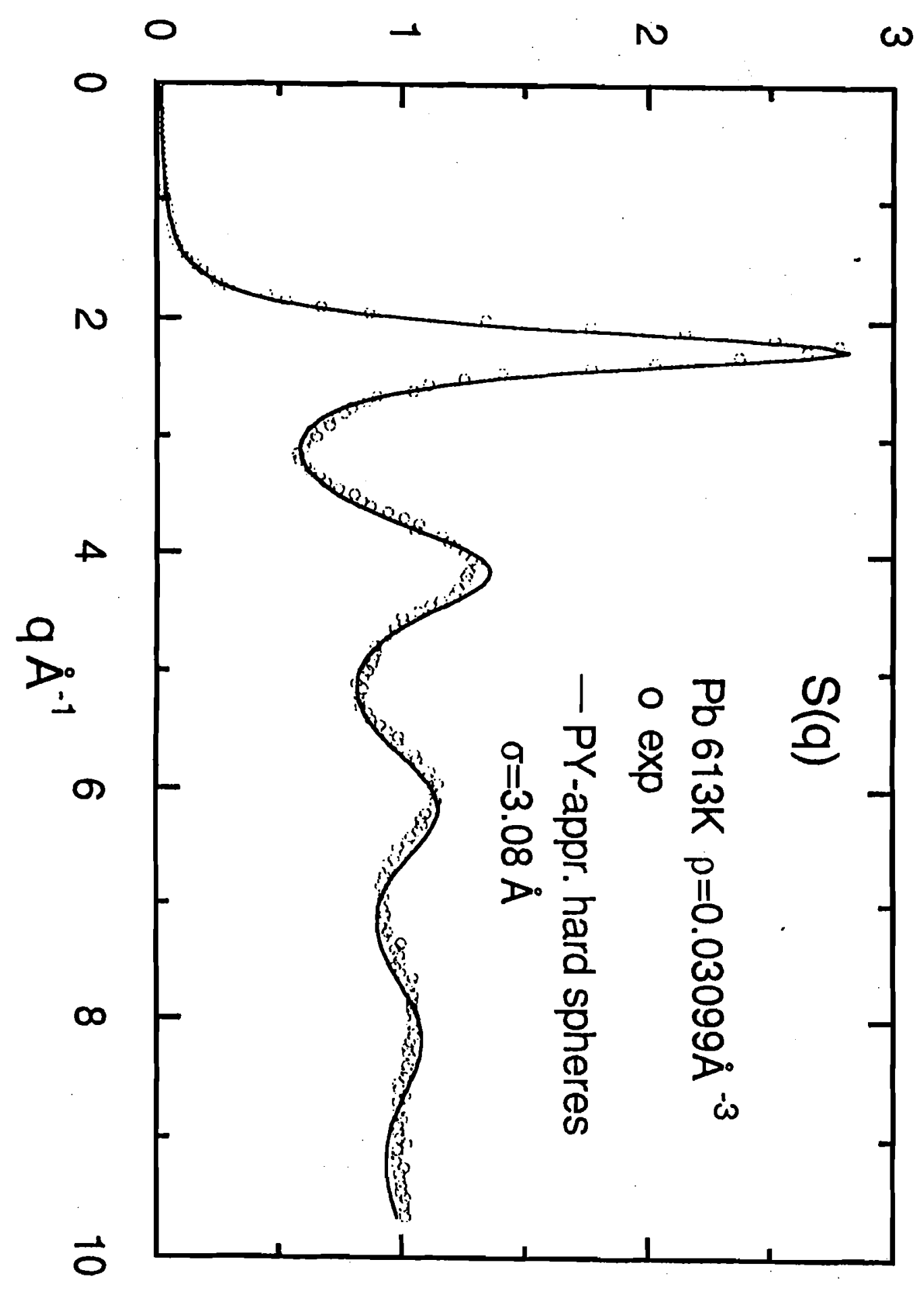




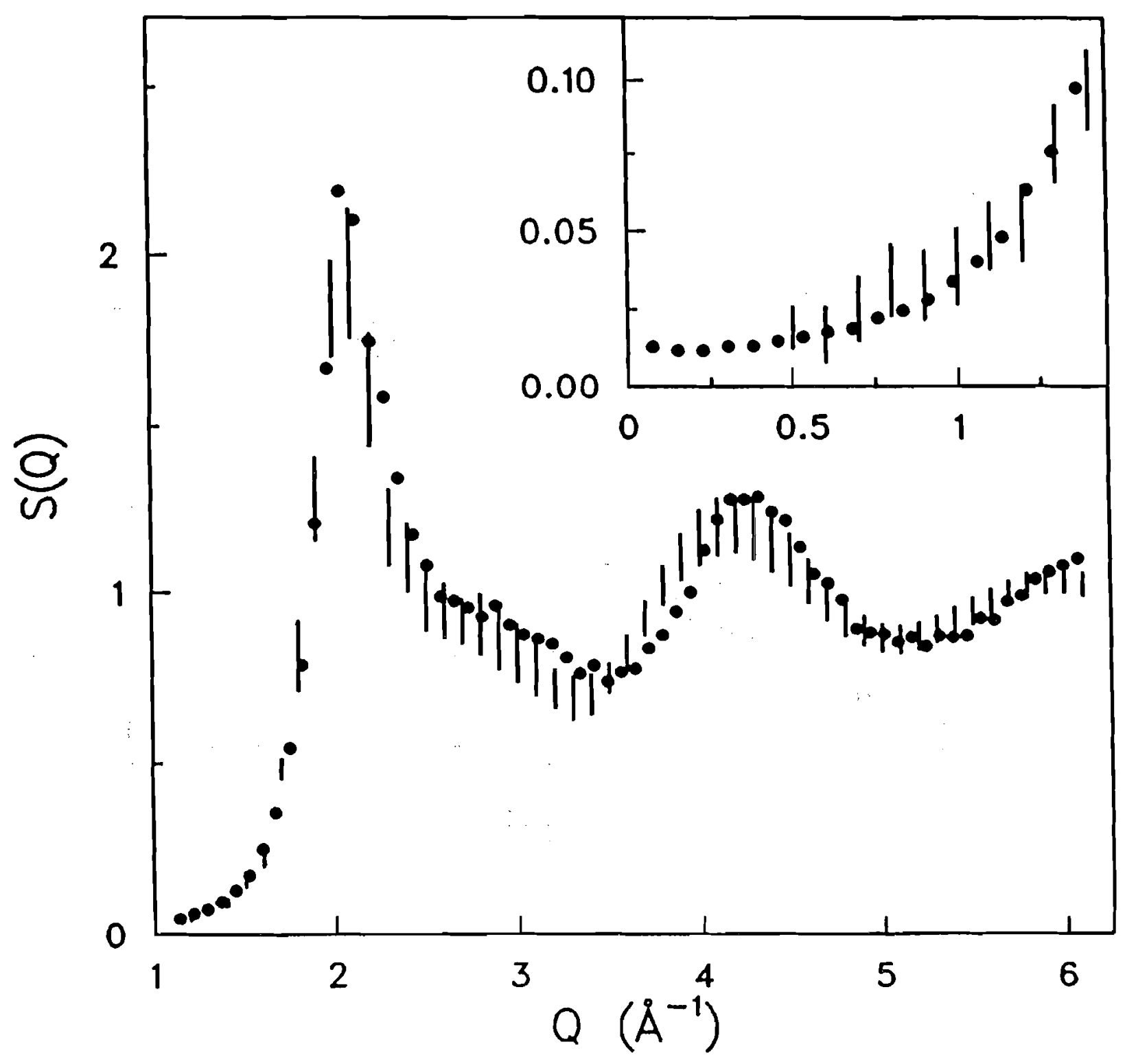

Fig 5 
9 b्u

(sd) $t$

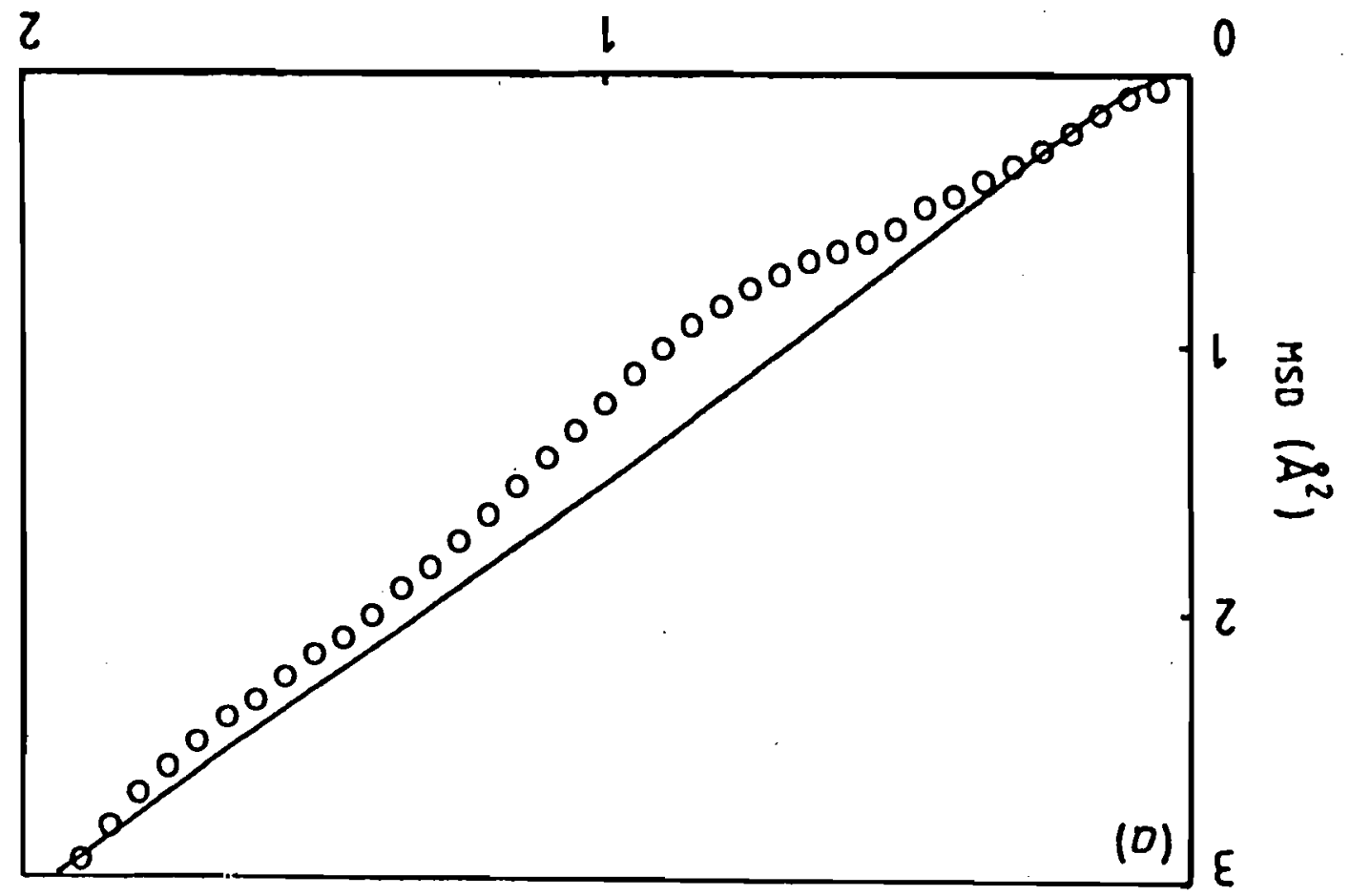



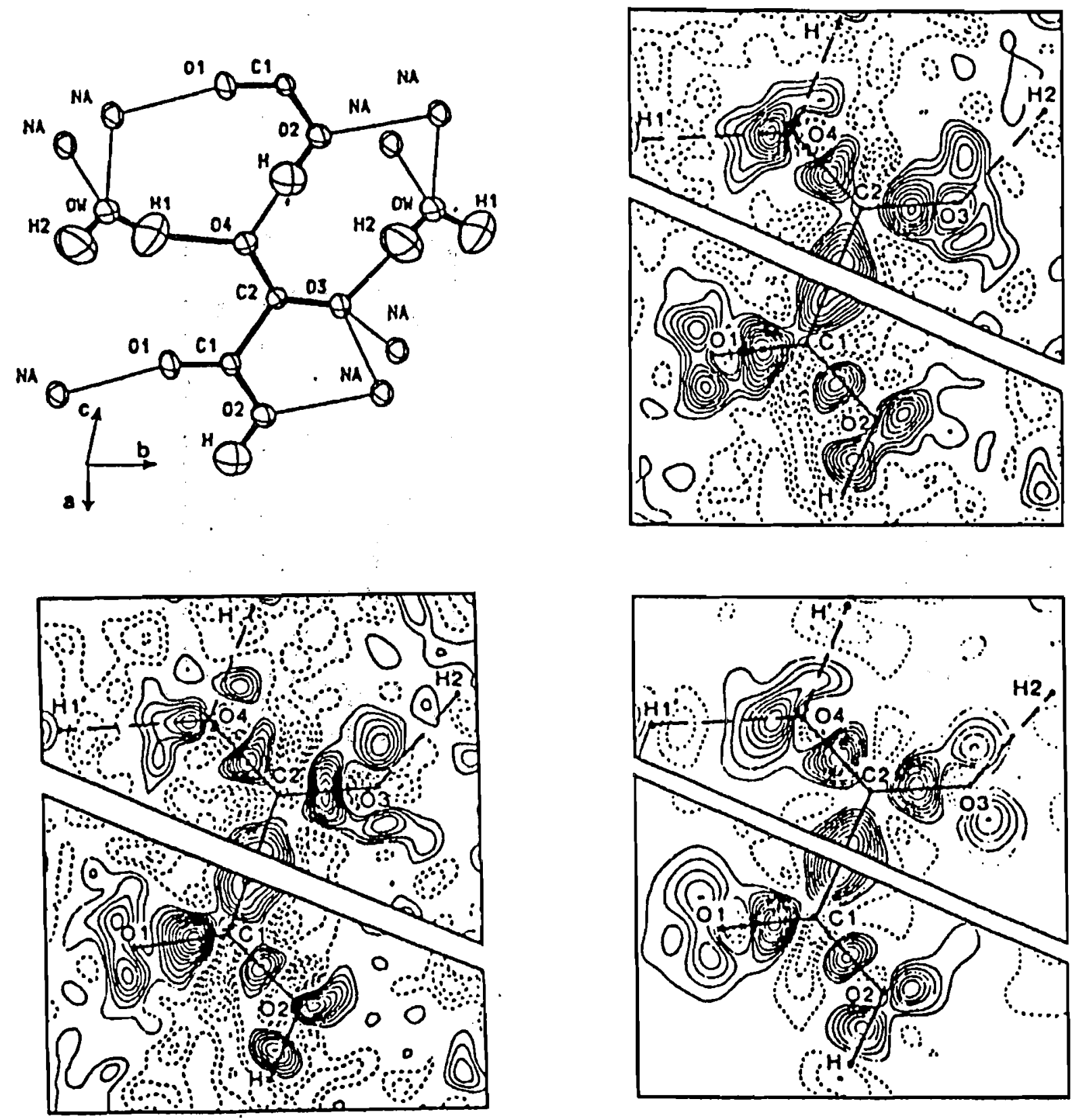
8 占出

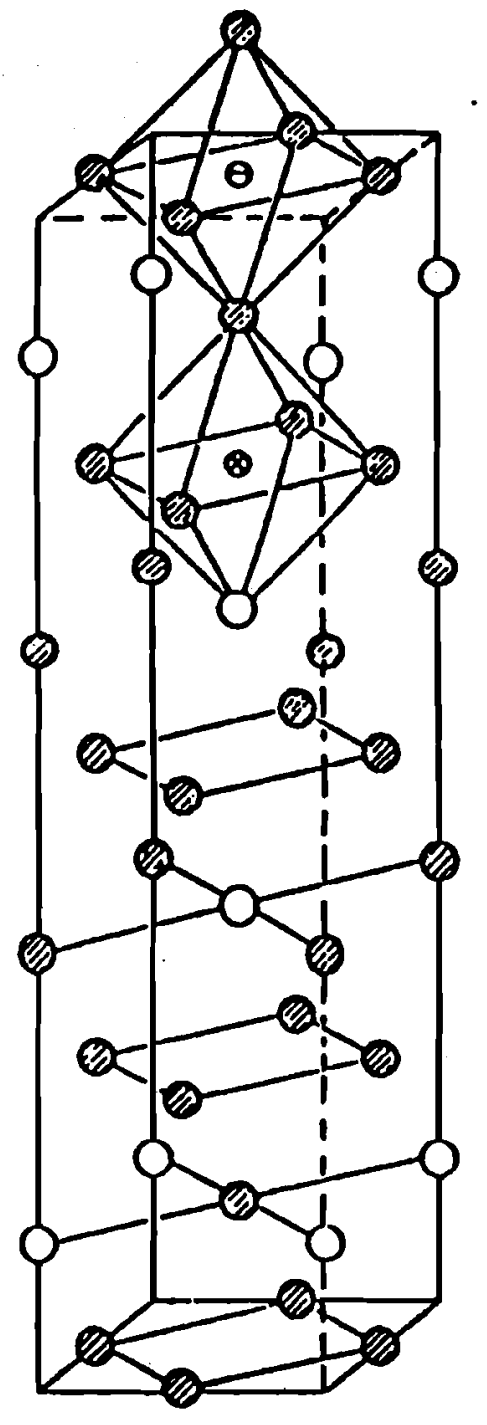

(2) $\mathrm{d} \otimes$

(1)d $\theta$

uW 0

pd 


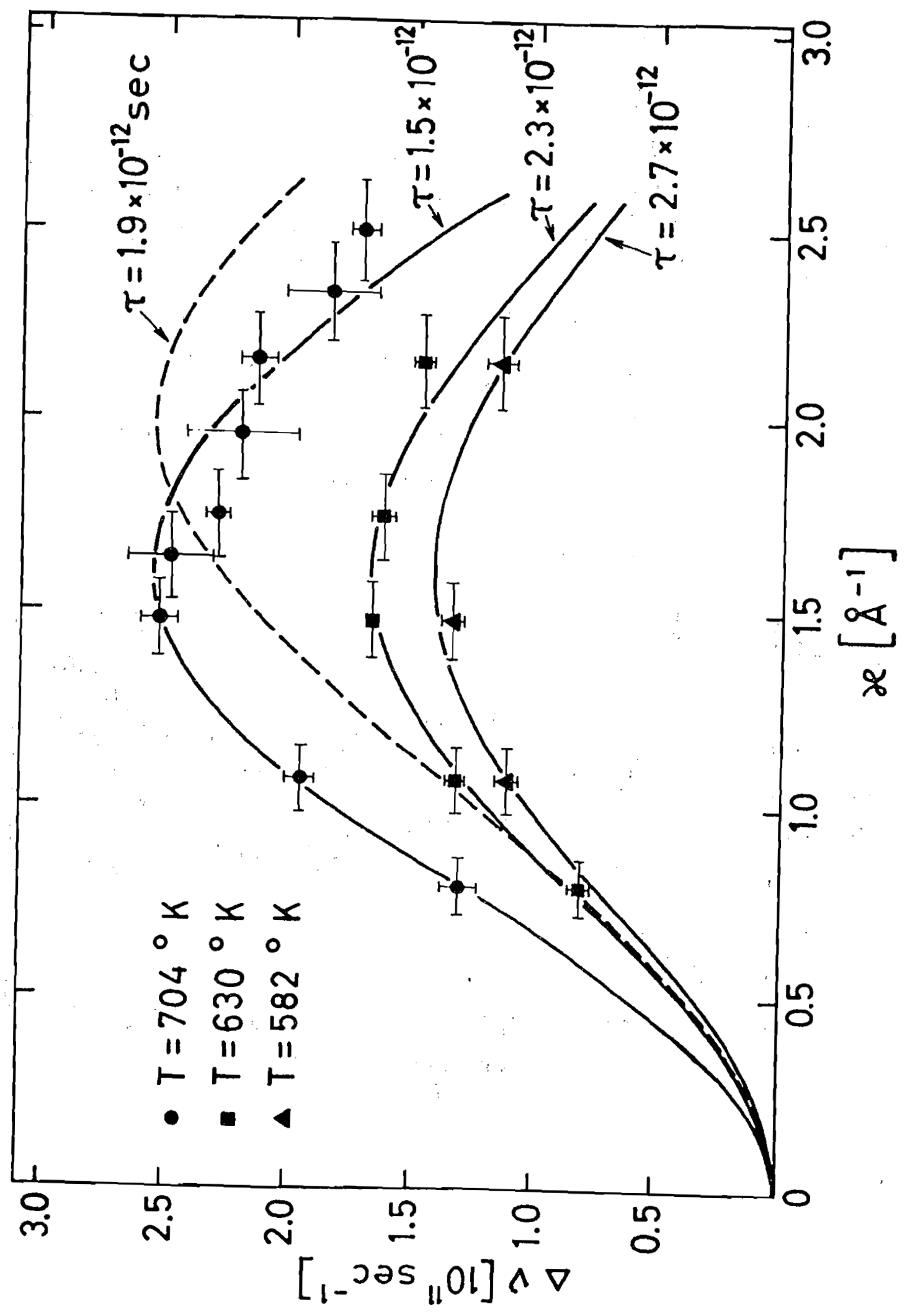

Fig 9 
o) 占办

(q)

(1- Y) O YヨISNYYI WNIN3WOW

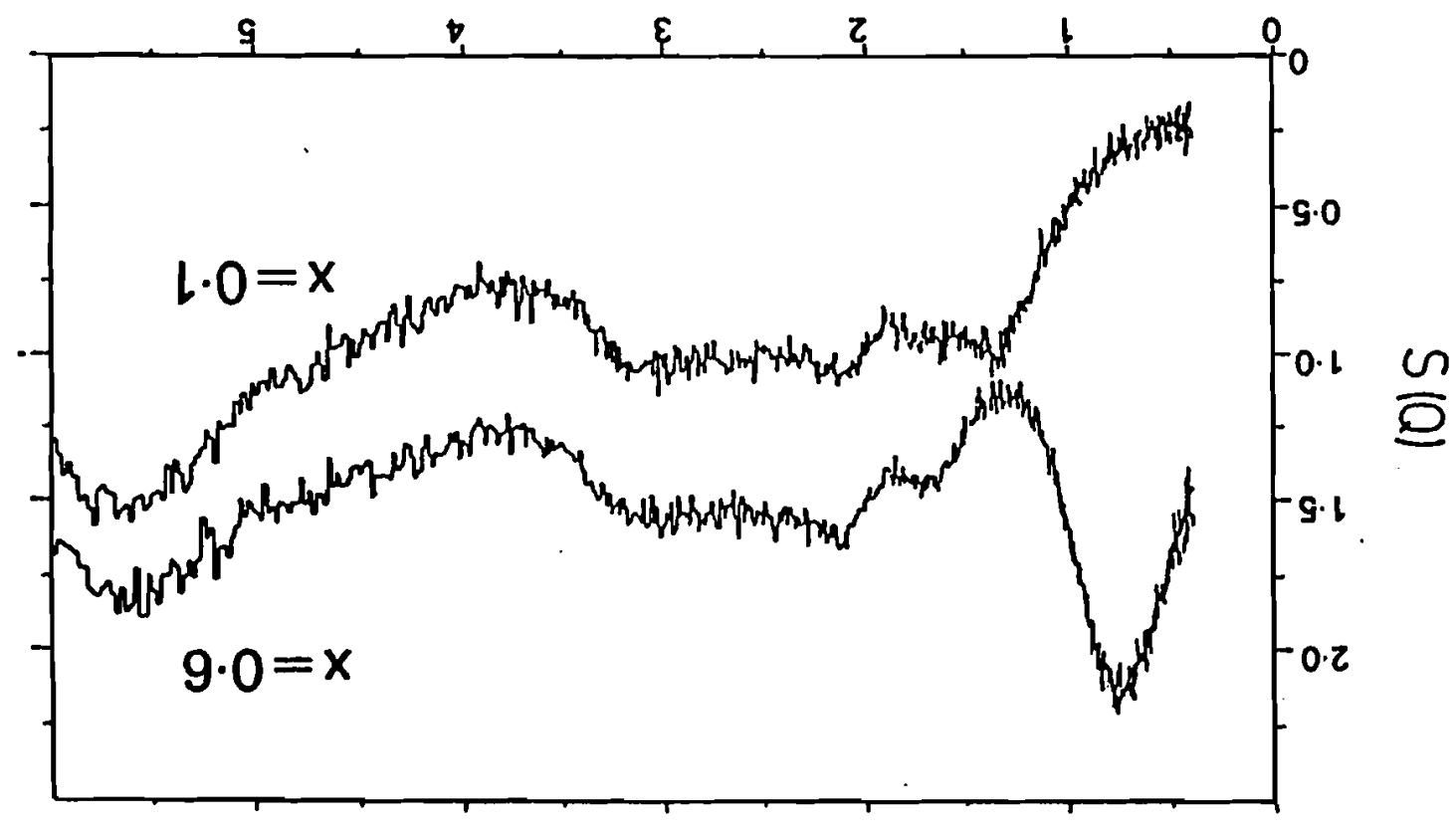

(n)

$(1-Y) O$ \&3ะSNYYI WกINGWOW

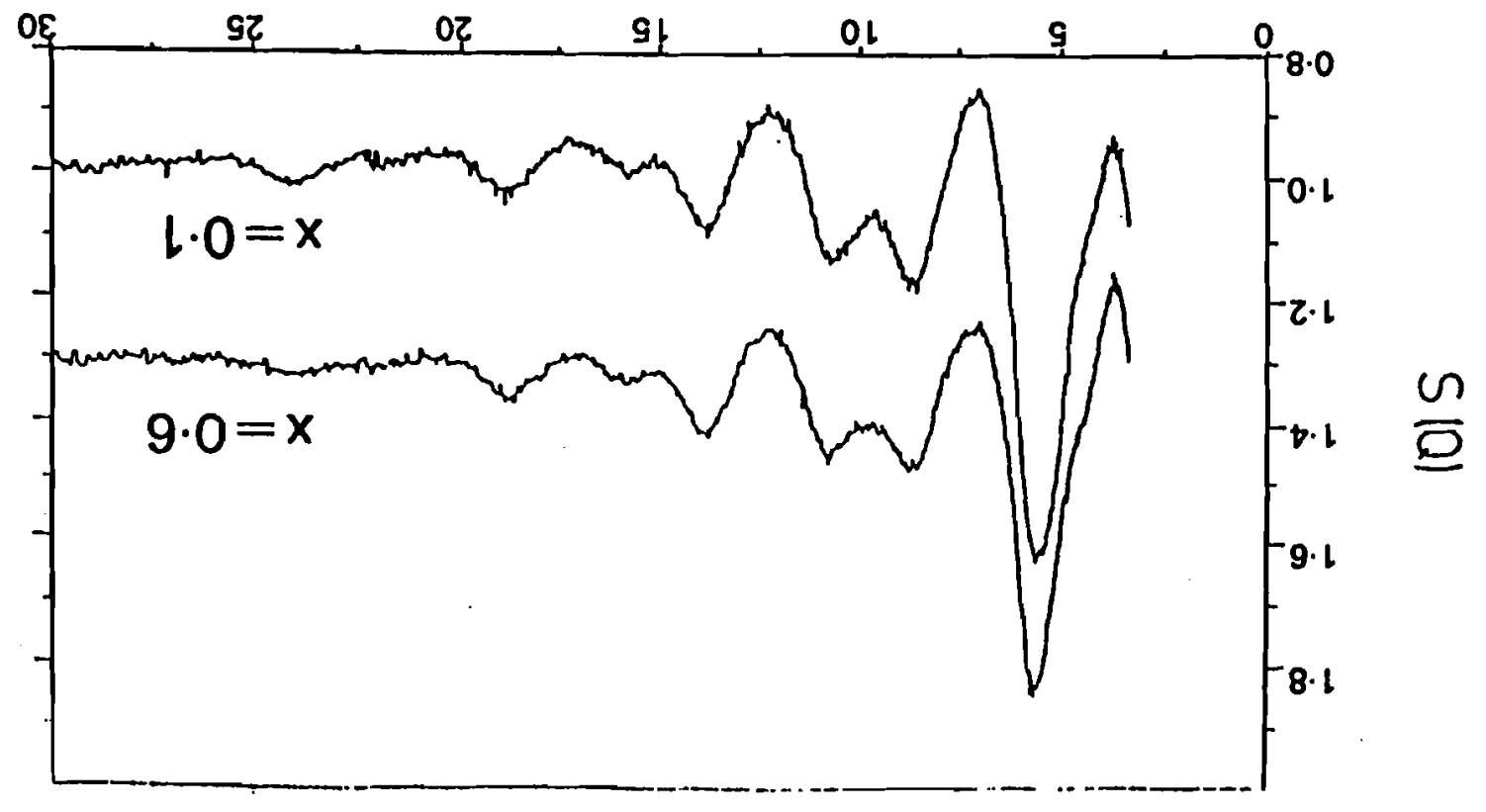




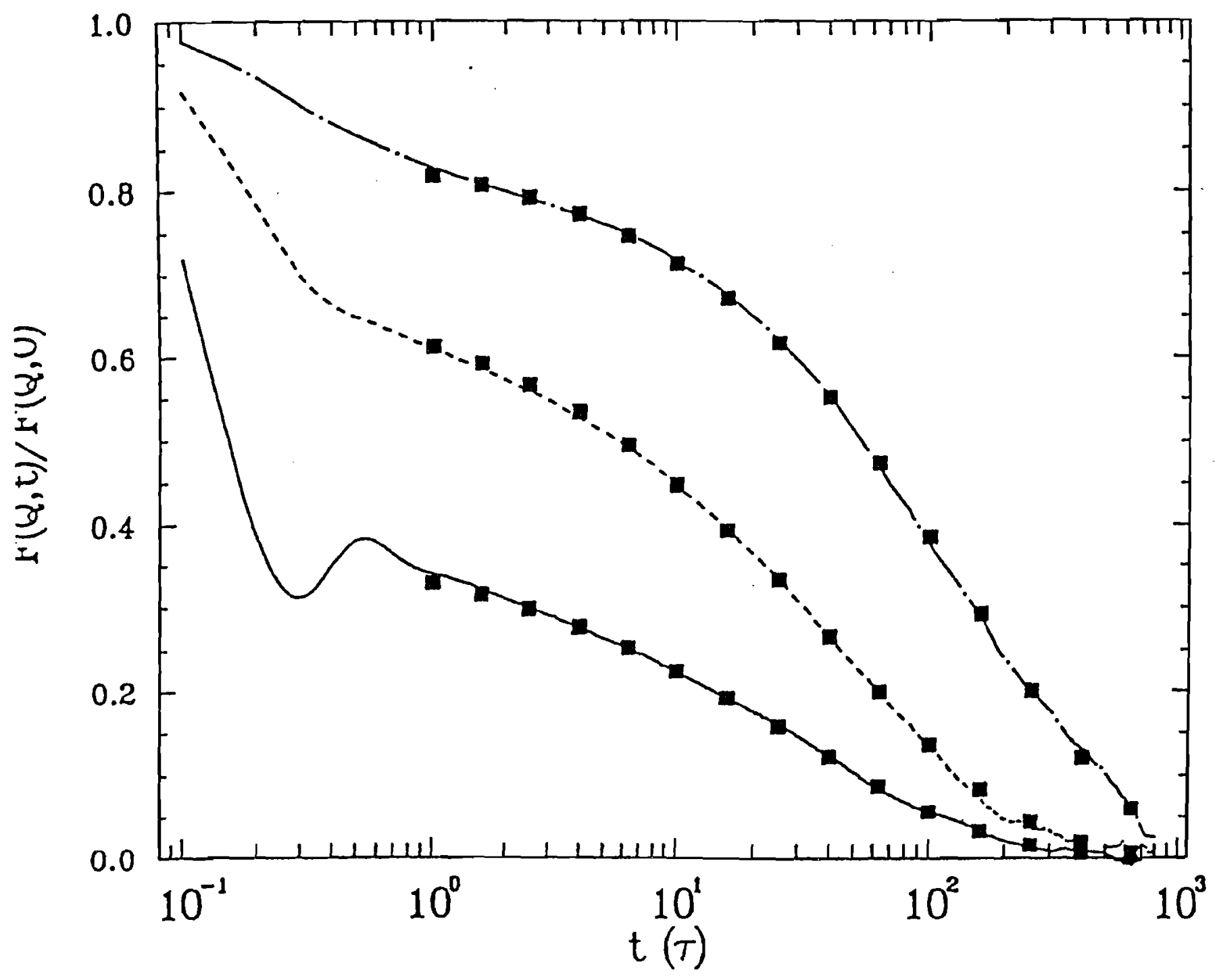

$\overline{T_{j}} "$ 


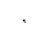




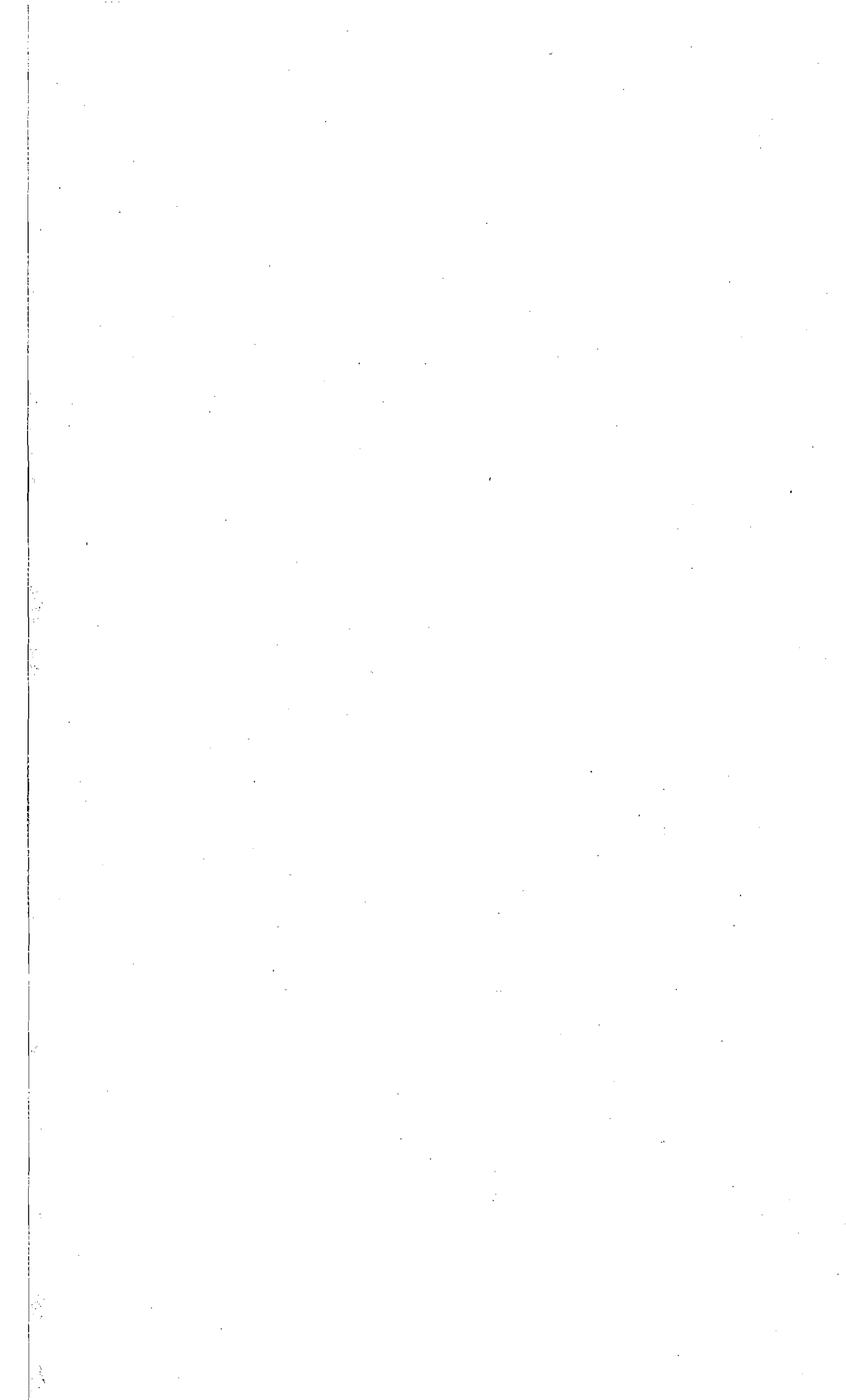




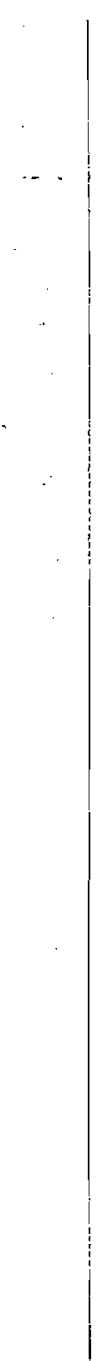


Science and Engineering Research Council

Rutherford Appleton Laboratory

Chilton DIDCOT Oxon OX11 0QX

RAL-90-077

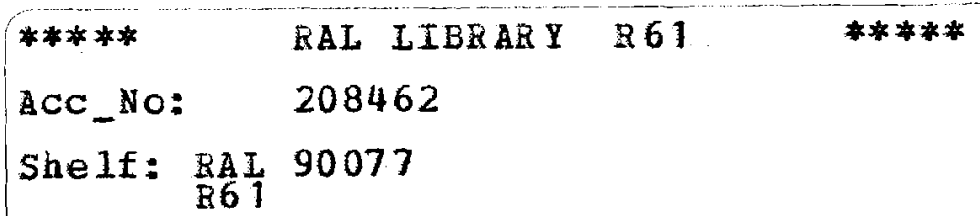

\section{Physics and Chemistry of materials} from Neutron Diffraction and Spectroscopy

Ulf Dahlborg and Stephen W. Lovesey

November 1990

$$
\begin{aligned}
& \text { LIRARY } \\
& R U T H=R D \\
& 10 \text { DEC } 1990
\end{aligned}
$$

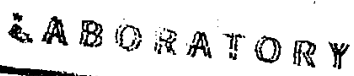


Science and Engineering Research Council

"The Science and Engineering Research Council does not accept any responsibility for loss or damage arising from the use of information contained in any of its reports or in any communication about its tests or investigations" 


\title{
PHYSICS AND CHEMISTRY OF MATERIALS FROM NEUTRON DIFFRACTION AND SPECTROSCOPY
}

\author{
by \\ ULF DAHLBORG \\ Institute of Physics, \\ Department of Neutron and Reactor Physics,
}

Royal Institute of Technology, S-100 44, Stockholm, Sweden

and

STEPHEN W. LOVESEY

Rutherford Appleton Laboratory,

Oxfordshire, OX11 OQX, England

and

Institute of Physics,

Uppsala University, S-751 21, Uppsala, Sweden 


\section{Abstract}

A short introduction to the powerful techniques of neutron diffraction and spectroscopy is illustrated largely with achievements by Swedish researchers in the past few years. Background material on sources and instrumentation is included, together with a directory of facilities routinely available to the Swedish scientific community. 



\section{Prologue}

Scattering experiments are important in many branches of chemistry and physics research. In condensed matter and materials research the projectiles used include atomic ions, electrons, neutrons and photons. While some experiments, like laser spectroscopy and x-ray diffraction, require a modest financial outlay, most demand access to major experimental facilities. The construction and operational costs are such that there is a trend toward central domestic and multi-national facilities, e.g. within Europe there is the neutron reactor source at the Institut Laue-Langevin, and the European Synchrotron Radiation Facility under construction next door to the ILL in Grenoble.

Multi-national facilities are often particularly well-funded and provide advanced instrumentation, but over-demand frequently forces discrimination against timeconsuming complex experiments and training exercises. These are two of the major reasons why, more modest, domestic facilities like the $\mathrm{R} 2$ reactor at Studsvik are essential. Another is that optimal use of multi-national facilities is only possible initial feasibility studies. Very often the information obtainable from a neutron scattering experiment is not available from other experimental techniques. The host of valuable and unique features of the neutron scattering technique vindicate the cost of building and operating neutron beam facilities.

Neutron diffraction studies of crystals and disordered systems provide structural information which complements $x$-ray results since, for non-magnetic materials, neutrons scatter predominantly from nuclei. The strength of this scattering varies quite strongly from one isotope to isotope, in an almost random manner, so contrast studies using isotopic substitution are widely used and particularly with hydrogenous materials. Magnetic diffraction provides information on both the configuration and distribution of the magnetization density. Neutron spectroscopy is widely used for the determination of; (a) dispersion curves of collective excitations in crystals (phonons), liquids and magnetic materials (spin waves); (b) localized, non-dispersive excitations such as hydrogen vibrational states in hydrides and macromolecules and atomic and crystal field states in magnetic materials; (c) diffusion of protons and (d) proton motions in 
macromolecular (polymer) systems.

The intention of this paper is two-fold. First, to serve as a brief introduction to neutron scattering (diffraction and spectroscopy) in investigations of properties of matter on an atomic scale. Emphasis is placed on results deduced from the interpretation of experiments rather than experimental methods, instrumentation, and data analysis. In view of the limited space available, an attempt is made to reference recent review articles, books and conference proceedings.

Secondly, the aim is to provide a perspective of Swedish neutron scattering work in chemistry and physics, and the facilities currently available to the Swedish science community. To this end, access to instrumentation on the $\mathrm{R} 2$ reactor at Studsvik, and the pulsed neutron facility ISIS at the Rutherford Appleton Laboratory, is described in an annex together with cameos of various spectrometers. Here again, the information we can provide is necessarily limited. The reader interested in performing experiments should consult the reference manuals listed and one of the personnel named in the annex.

In many respects the Swedish scientific community is privileged as regards access to neutron scattering facilities. The domestic reactor facility at Studsvik has recently been refurbished with large beam tubes. By the end of 1990 there will be a suite of instruments suitable for a range of diffraction and spectroscopic studies of crystals, powders, amorphous materials and liquids. Instrumentation on the ISIS facility is largely complerrientary, giving access to large ranges of energies and wavelengths. The very large signal-to-noise ratio available on ISIS instruments means that the resolution attainable in several cases is unmatched by similar instruments placed at reactors.

The new user can draw on a store of experience held by Swedish scientists who have worked at the forefront of neutron scattering research. Admittedly the experience is predominantly with nuclear scattering, but studies of magnetic phenomena are now planned.

Basic properties of the neutron and a sketch of its discovery are provided in sections 2 and 3, respectively. A broad based review, which includes a listing of neutron 
properties, historical notes and a survey of techniques and sources has been prepared by Scherm [1]. Here, neutron sources are briefly reviewed in section 4 and the basic concepts required for the interpretation of experiments are outlined in section 5 . Theoretical work is underpined by the framework of linear response theory [2], which acts also as a unifying language with the interpretation of many other experiments such as NMR, Mössbauer, $\mu$ SR, and light scattering [2]. Illustrative examples of neutron beam research, with a strong slant towards work involving Swedish scientists and facilities, are provided in section 6 . In view of this selection the examples are mostly studies in which nuclear scattering is used. However, the material in section 5 on magnetic scattering should hopefully give the reader an inkling as to the power of neutron scattering in studies of magnetic phenomena. Reviews of the relevant theory and experiments can be found in references $[2,3,4]$. Our speculation on future trends in neutron beam research is given in section 7 .

\section{Neutron Properties}

The utility of neutron scattering as an atomic-scale probe of condensed matter stems from the relative weakness of neutron-matter scattering, the compatibility of neutron energies and wavelengths with characteristic energies and lengths of atomic motions in solids and liquids, and the magnetic moment of the neutron. The neutron-matter interaction is so weak that first-order perturbation theory is wholly adequate to account for the neutron scattering cross-section. In other words, neutron scattering provides information on the chemical and physical properties of matter that is undistorted by the radiation. Furthermore, the interpretation of the measured cross-section is not clouded by uncertainty about the nature of the radiation-matter interaction or specification of the cross-section.

Neutrons interact with the nuclei and electrons in matter, and the scattering crosssections are similar in magnitude. It is possible to discriminate between scattering events involving nuclei and electrons. In consequence, the neutron scattering technique provides unambiguous information on the positions and motions both of nuclei and of electrons. Moreover, since neutrons penetrate deeply into matter they provide an ideal probe of the bulk properties of matter [1-4]. However, neutrons are also absorbed in matter. 
Neutron nuclear scattering as well as the absorption cross sections vary from one isotope to isotope in a more or less random manner. In many applications it is particularly advantageous that the scattering cross sections for the proton and deuteron are very different. The cross section for a proton is a massive 82 barns ( 1 barn $=10^{-24}$ $\mathrm{cm}^{2}$ ) whereas for a deuteron it is an order of magnitude smaller. Hence, the proton furction in macromolecules, membranes, etc, is readily studied, while deuteration enables us to pick out properties of the host environment. The magnitude of neutron scattering and absorption cross sections for some selected nuclei are given in table 1.

The energy $E$ of a neutron with a wave vector $k$ is

$$
E=\hbar^{2} k^{2} / 2 m,
$$

where $\mathrm{k}=|\mathbf{k}|$ and $\mathrm{m}$ is the neutron mass. Energies are often given in units of $\mathrm{meV}=$ $10^{-3} \mathrm{eV}$, and $\left(\hbar^{2} / 2 \mathrm{~m}\right)=2.08 \mathrm{meV} \AA^{2}$. In terms of the neutron wavelength $\lambda$ we have,

$$
\lambda=\left(h^{2} / 2 \mathrm{mE}\right)^{1 / 2}=9.04 \mathrm{E}^{-1 / 2},
$$

where $\lambda$ is in $\AA$ and $E$ is in meV. Here we have chosen the energy unit favoured by physicists. Other energy units frequently used in spectroscopy are related to the meV through

$$
1 \mathrm{meV} \equiv 0.24 \mathrm{THz} \equiv 8.07 \mathrm{~cm}^{-1} \equiv 11.61 \mathrm{~K}
$$

and the conversion to temperature is included for completeness.

It is perhaps useful at this junction to recall that in contrast to neutron scattering from materials photon scattering is dominated by the photon-electron interaction, described by quantum electrodynamics. Absorption of photons due to the photo-electric process and Compton effect severely reduce photon penetration in matter, and so the technique is highly surface sensitive. The photon-matter interaction contains complicated processes, some of which involve nonlinear events not describable within the framework of linear reponse theory that underpins the interpretation of neutron scattering (excluding events that involve compound nuclear resonance states). 
The high intensity of photon beams from synchrotron sources makes it feasible to exploit magnetic photon scattering ( a relativistic correction to the Thompson amplitude) as a probe of condensed matter [5]. This technique has some advantages with respect to magnetic neutron scattering, although it is likely to be confined to elastic (diffraction) and Compton scattering studies for the most part in the near future.

By and large, selection rules, which are manifestations of high symmetry conditions, operate more forcefully in photon scattering than in neutron scattering. In optical spectroscopy, for example, the change in photon wave vector, $k$, is so small that events obey the dipole selection rule, whereas in a corresponding neutron induced event $\mathbf{k}$ can be relatively large and additional processes are engaged, c.f. section 5.2. Similar reasoning holds for the excitation of states in a crystal. Selection rules are manifest at points of high symmetry in the Brillouin zone, such as the zone centre and boundary, and generally absent at an arbitrary point on a dispersion curve.

\section{History}

Joliot-Curie's communication of 28 January 1932, reported that alleged $\gamma$-rays from the $\alpha$-beryllium reaction were capable of ejecting protons from paraffin. When the paper reached Chadwick, at the Cavendish Laboratory, he went to work and on 17 February submitted a paper entitled 'possible existence of a neutron' in which he proposed that the $\alpha$-beryllium reaction is $\alpha+\mathrm{Be}^{9}=\mathrm{C}^{12}+n$. Chadwick's discovery of the neutron, in a few days of strenuous work, concluded a search that, off and on, had been conducted at the Cavendish for more than a decade.

In early 1934 Fermi submitted the first in a series of articles on radioactivity induced by neutron bombardment. With this paper Fermi started experimental studies in neutron physics that made him perhaps the world's leading authority on the subject during the nineteen thirties. His pseudopotential method, applied to neutron scattering by nuclei in condensed matter, appeared in 1936 and it remains the cornerstone of the interpretation of low energy neutron-nucleus scattering experiments.

In the same year Bloch predicted that the electromagnetic neutron-electron amplitude is similar in magnitude to the classical electron radius, and therefore comparable to 
nuclear scattering amplitudes. The following year Schwinger queried Bloch's calculation: the latter is now known to be incorrect, and Schwinger provides the correct result although his reasoning is erroneous. A correct calculation, and physical interpretation, is provided by Migdal in a paper submitted in July 1938 to a Russian Journal. However, Migdal's work was unnoticed in the West, and the Bloch-Schwinger controversy was finally settled: in 1951 when two independent experiments found unambiguous evidence to support the result given by Schwinger. The basis of the interpretation of neutronelectron scattering experiments is the paper by Halpern and Johnson published in 1939. (Although these authors subscribe to the Schwinger view they nevertheless propose experiments to settle the Bloch-Schwinger controversy.)

The major sources of neutrons in the early nineteen thitties were radium-beryllium sources. Even though it was possible to demonstrate the diffractive properties of neutrons, the low intensities from radium-beryllium did not permit the practical use of neutron beams to study the properties of condensed matter. The latter began to flourish with the development of nuclear reactors. The Oak Ridge Graphite Reactor and the CP-3 reactor at the Argonne National Laboratory became operational in 1943 and 1944 , respectively.

Neutron intensities produced by modern, high-flux reactors are three orders of magnitude larger that those obtained with the early reactors at the Oak Ridge and Argonne Laboratories. Moreover, new vistas are now being opened with advanced spallation sources which utilize protons, accelerated in a synchrotron, to liberate a very large supply of energetic neutrons from heavy metal targets.

\section{Neutron Sources}

The self-sustaining nuclear fission reaction has been used as the basis for most neutron sources primarily constructed for scattering experiments. The effective thermal neutron fluxes available from steady-state reactors has steadily improved over the past 40 years, and a saturation level has been reached at approximately $1.2 \times 10^{15} \mathrm{n} / \mathrm{cm}^{2} \mathrm{~s}$, the flux provided by the high-flux reactor at the Institut Laue Langevin (ILL), Grenoble. 
The practical limits in this flux are imposed by a combination of engineering constraints, due to the large power density in the core, as well as to economic factors. Plans are underway to construct a new high flux reactor at the Oak Ridge National Laboratory, USA, which has a flux increase of the order 2-4 over the ILL reactor; this will require the solution of new technological problems and an investment in the region of 2000 MSEK. Future improvements at existing reactor sources will result from upgrading their power and their associated experimental facilities, and a review of current proposals has been published by Moon and West [6]. It is nevertheless generally agreed that the practically attainable flux from a steady state reactor has reached its limit.

Pulsed neutron sources are able to provide instantaneous neutron fluxes which are higher than those from continuous reactors. Since they only operate for a fraction of a time period, their momentary power densities and neutron fluxes can be very high, while their average power densities can be kept at modest levels compatible with ease of heat extraction. Practical pulsed sources for neutron scattering can be either pulsed reactors or accelerator-based systems in which the burst of fast ( $\mathrm{MeV})$ neutrons is moderated in a hydrogenous moderator to provide the effective source for the experiments. The proton spallation process is the most competitive accelerator-based neutron source to date; it has the highest neutron yield per incident particle and also the lowest energy dissipation in the target per neutron generated. Typical values of these parameters for the most frequently used neutron-producing reactions are summarized in table 2 together with corresponding target powers for existing sources.

An important parameter, perhaps most relevent one when discussing the possibilities to remove energy in a neutron-producing facility, is the power density. For the ILL reactor this is about $600 \mathrm{~kW} / \mathrm{l}$ while the corresponding figure for ISIS is about $75 \mathrm{~kW} / \mathrm{l}$ which indicates that the spallation sources by no means have reached its maximum strength with the advent of ISIS: A pulsed beam imposes, however, a higher demand on the efficiency of energy removal.

As a final topic in this section we mention that a pulsed reactor has been constructed at Dubna, USSR. Fission occurs during the short time that a rotating reflector blade (rotational frequency $5 \mathrm{~Hz}$ ) passes a ${ }^{239} \mathrm{PuO}_{2}$ target, and the average power generated is approximately $4 \mathrm{MW}$ giving an impressive instantaneous thermal neutron flux of order 
$10^{18} \mathrm{n} / \mathrm{cm}^{2} \mathrm{~s}$. The major disadvantage is the inherently long pulse width (approximately $250 \mu \mathrm{s}$ after moderation) so that only relatively poor resolution experiments are possible, particularly with short wavelength neutrons. There is also the potential hazard associated with moving substantial mechanical objects to a high degree of reliability in an intense radiation environment.

\section{Baslc Concepts}

Since the scattering of slow neutrons is a weak process it can be described by firstorder perturbation theory, i.e. Fermi's Golden Rule for transition rates. In consequence, we treat the incident and scattered neutron states as plane waves with energies $E, E^{\prime}$ and wave vectors $\mathbf{k}, \mathbf{k}^{\prime}$, related as in eqn (1). The cross-section, or response function, is described in a four dimensional space spanned by the variables

$$
\hbar \omega=E-E^{\prime}
$$

and

$$
\mathbf{Q}=\mathbf{k}-\mathbf{k}^{\mathbf{\prime}}
$$

If the target sample is spatially isotropic the response function depends orly on $\omega$ and $Q=|Q|$.

The differential cross-section, which has the dimension of (area/energy), is readily expressed in terms of correlation functions that are determined solely by the chemical and phiysical properties of the target sample. This is by far the most elegant and powerful representation, and the one adopted here. Further details, including an exposition of linear response theory, are found in [2] and the theory chapters in [3,4]. We will separately describe nuclear and magnetic scattering, though some basic concepts are common to both, of course. Although polarization phenomena are important, and more widely used today with improved sources and instrumentation, we do not include it in the basic theory because this is intended very much as an introduction and therefore it should be kept as simple as is reasonably possible. Full accounts of polarization phenomena are provided in references $[2,7]$. 


\subsection{Nuclear Scattering}

Slow neutron scattering is described by a single parameter, namely a scattering length $b$ which is assumed to have minimal energy dependence. The imaginary part of $b$ gives rise to absorption. This is extremely large for some isotopes, e.g. about 15000 barns for $\mathrm{He}^{3}$ and $5 \AA$ neutrons.

The nuclear scattering cross-section is a scattering length weighted sum of correlation functions. Let $\mathrm{i}, \mathrm{j}$ label the scattering nuclei, and denote the correlation function by $Y_{1]}(Q, t)$. With this notation the partial differential cross-section is,

$$
\frac{d^{2} \sigma}{d \Omega d E^{\prime}}=\left(E / E^{\prime}\right)^{1 / 2} \int d t(1 / 2 \pi \hbar) e^{-t \omega t} \sum_{i, j} b_{1}^{*} b_{j} Y_{l j}(Q, t),
$$

where $d \Omega$ is the solid angle subtended by the detector. The function $Y_{11}(Q, t)$ possesses properties that make the right-hand side of (5) both real and either positive or zero, as required for a response function. It is necessary to average the cross-section over the distribution of nuclear spins (assumed to be completely random), isotopes, substitutional disorder, etc. We will deal with these averages as and when required.

5.1.1. Purely elastic scattering. This is generated by the value of the correlation function at infinite time; $Y_{1}(Q, \infty)$ with $Q \neq 0$ is finite for crystals and fully arrested super-cooled liquids, for example, but vanishes for normal liquids. For a crystal, it is customary to write

$$
Y_{i j}(\mathbf{Q}, \infty)=\exp \left\{i Q \cdot\left(\mathbf{R}_{j}-\mathbf{R}_{i}\right)-W_{j}(\mathbf{Q})-W_{i}(\mathbf{Q})\right\}
$$

where $\mathbf{R}_{i}$ is the equilibrium lattice position of the $i^{\prime}$ th atom and the remaining terms are Debye-Waller factors. An explicit expression for $W(Q)$ valid for a harmonic lattice is given following eqn (16).

Bragg scattering is elastic and coherent and occurs under special geometrical conditions. It is generated by a perfect crystal, which means that the appropriate crosssection is formed with the square of the average of the effective scattering length per 
unit cell. Let the crystal contain $\mathrm{N}$ unit cells of volume $\mathrm{v}_{\mathrm{o}}$ in which the atoms are at sites defined by position vectors $\mathrm{d}$. The elastic coherent cross-section is then

$\left.\stackrel{d \sigma}{\left(\frac{\mathrm{d} \Omega}{d \Omega}\right.}\right)_{\text {coh }}^{\text {el }}=N(2 \pi)^{3} / v_{0} \sum_{\tau}\left|F_{N}(\tau)\right|^{2} \delta(Q-\tau)$

in which $\{\tau\}$ are reciprocal lattice vectors and the unit cell structure factor

$F_{N}(Q)=\sum_{d} \bar{b}_{d} \exp \left(i Q \cdot d-W_{d}(Q)\right)$

where $\bar{b}$ is the scattering length averaged over isotope and nuclear spin distributions. From (7) it is evident that scattering occurs only when the condition $\tau=Q$ is satisfied, which is a statement of Bragg's Law.

The difference between the total elastic scattering and the Bragg intensity from a solid is due to disorder and defects in the crystal, nuclear spins and mixtures of isotopes. By definition it is not coherent and it occurs to some exterit at all scatteririg angles. It is customary, but not completely logical, to use the term incoherent scattering for nonBragg scattering generated by nuclear spins and isotope mixtures (note that a sample of a pure isotope can produce incoherent scattering if the nuclear spin is finite, e.g. $\mathrm{He}^{3}$ ). All the remaining non-Bragg scattering is called diffuse scattering.

Incoherent elastic scattering from a crystal measures the quaritity,

$$
N \sum_{d}\left[\overline{b_{d}^{2}}-\left(\bar{b}_{d}\right)^{2}\right] \exp \left[-2 W_{d}(Q)\right]
$$

This can be interpreted as the sum over atoms in the unit cell of the Debye-Waller factor weighted by the mean-square fluctuation in the scattering length.

As an example of elastic diffuse scattering consider a binary system in which type-2 atoms (impurity atoms, say) occur with concentration c. All other things being equal apart from a difference in coherent scatterirg lengths, diffuse scattering occurs which is proportional to, 
A more realistic model would allow for the difference in Debye-Waller factors and the deformation in the host lattice created by the impurity atoms. In the latter case, the appropriate cross-section is proportional to the absolute square of the spatial Fourier transform of the deformation, which can be compared to theoretical predictions.

5.1.2. Total scattering. For a liquid strictly elastic events occur only if $Q=0$, which corresponds to no scattering. Hence, the total scattering is measured in a neutron scattering experiment without energy analysis. This quantity is thus the response function integrated over all neutron energy transfers. With a monatomic sample, in which quantum effects are negligible (achieved with large $A$ atoms at relatively high temperatures), the total coherent scattering, at constant $Q$, is proportional to the structure factor,

$$
S(Q)=1+\rho_{0} \int d r e^{i o r}[g(r)-1]
$$

in which $\rho_{0}$ is the particle density and $g(r)$ is the pair distribution function. It is, perhaps, useful to note that $r^{2} g(r)$ is the probability distribution for the particle density about the origin. Hence, the number of particles within a sphere of radius $R$ prescribed about a given particle is

$$
4 \pi \rho_{0} \int_{0}^{R} d r r^{2} g(r)
$$

In the limit $R \rightarrow \infty$ this quantity approaches the total number of atoms in the sample, as required.

Before turning to inelastic scattering we draw attention to a basic difference between Bragg scattering and total scattering. The latter is readily shown to be proportional to $Y_{j i}(Q, 0)$, i.e. the instantaneous value of the correlation function. On the other hand, Bragg scattering is proportional to the square of a time-averaged variable (a basic principle in statistical physics is that statistical averaging is completely equivalent to time averaging). The difference between these two extreme limits of the correlation function is related to the appropriate isothermal susceptibility, i.e. the mean-square fluctuation 
in the number density. We conclude that the difference is small except in the vicinity of a phase transition, when fluctuations take macroscopic values. One final point to make is that total scattering is often referred to as the static approximation to the crosssection, the choice of terminology being more or less self-evident in view of what we have just said.

5.1.3. Neutron spectroscopy. This corresponds to scattering events for which $\omega \neq 0$ and thus are termed inelastic. It is customary to use the label quasi-elastic for the part of the inelastic spectrum which arises from random, or stochastic, processes that occur over relatively long time scales [8], e.g. diffusive motion of an atom in a liquid. If classical statistics apply, the incoherent (single-particle) scattering cross-section is in this case determined by the correlation function

$$
Y(Q, t) \sim \exp \left(-Q^{2} D|t|\right)
$$

where $D$ is the self-diffusion constant. The corresponding cross-section is

$$
\frac{d^{2} \sigma}{d \Omega d E^{\prime}}=\left(\frac{E}{E^{\prime}}\right)^{1 / 2} \frac{\sigma_{1}}{4 \pi^{2}} \frac{Q^{2} D}{\omega^{2}+\left(Q^{2} D\right)^{2}}
$$

in which $\sigma_{i}$ is the incoherent cross-section (79.8 barns and 2.0 barns for a proton and deuteron, respectively). A similar result holds for a particle jumping between interstitial sites of a lattice, e.g. hydrogen diffusion in metals. In this instance $D=l^{2} / \tau$, where $l$ is a length, of order the lattice constant, and $\tau$ is the residence time at a given site.

The generic form of the correlation function that appears in quasi-elastic scattering is, to a good approximation,

$$
Y(Q, t)=\exp \left(-Q^{2} \mu(t) / 2\right)
$$

in which $3 \mu(t)$ is the mean-square displacement after a time $t$. The result $\mu(t) \sim|t|$ found for uncorrelated jumps on a lattice is characteristic of a random-walk process.

If a particle is bound in a crystal or macromolecule then often a useful starting point is to consider scattering from a harmonically bound particle. The scattering response for a particle with a natural frequency $\omega_{0}$ is, 


$$
\exp [-2 W(Q)+(1 / 2) \hbar \omega \beta] \sum_{n=-\infty}^{\infty} l_{n}(y) \delta\left[\hbar \omega-n \hbar \omega_{0}\right]
$$

The interpretation of (16) is straightforward; the scattering vanishes unless $\omega=n \omega_{0}$, and the integer $n$ measures the number of units of energy $\hbar \omega_{0}$ lost $(n>0)$ or gained $(n<0)$ by the neutron. The various quantities in (16) are; $B=1 / k_{B} T$ ( $T$ is the absolute temperature), $I_{n}(y)$ is a Bessel function of the first kind, and using the dimensionless variables $X=\left(\hbar \omega_{0} B / 2\right)$ and $\gamma=\left(\hbar Q^{2} / 2 M \omega_{0}\right)$ where $M$ is the mass of the scattering particle, $y=\gamma / \sinh (x)$ and $W=(\gamma / 2) \operatorname{coth}(x)$. Note that the elastic contribution $(n=0)$ contains $I_{0}(y)$. This factor arises from thermal fluctuations of the bound particle, which are negligible if the particle participates in a bulk collective motion.

The terms in (16) with $n= \pm 1$ are usually labelled the fundamental modes. If $y<<1$, as is often the case, the intensity of higher-order modes is very small in comparison with the fundamental since,

$$
I_{n}(y) \sim(1 / n !) y^{|n|} ; y \rightarrow 0
$$

In the limit $\left(\hbar Q^{2} / M \omega_{0}\right) \gg>1$, which can be achieved with a pulsed neutron source, it is not possible to select the fundamental mode in the response. A palisade of modes of near equal amplitude are engaged in scattering, so the expansion leading to the representation (16) is of minimal value. It can be shown that, in the limit of large $Q$, the scattering response tends to a Gaussian envelope function centred at the recoil energy $(\hbar Q)^{2} / 2 M$ with a mean-square width of $2 \omega_{0}{ }^{2} W(Q)$.

A far more realistic model of single particle dynamics is achieved by considering the particle as a defect in a host matrix. Two parameters characterize the particle matrix system, namely the ratio of the two masses and the deviation of the particle-matrix stretching force from that in the bulk matrix. The dynamics of the particle can be obtained in closed form if the stretching forces are harmonic. Calculations with this model reveal that a light mass (e.g. proton in a macromolecule) creates a high frequency mode which is well separated from the maximum phonon frequency in the pure host. By using a Debye model for the latter the frequency of the new mode is

$$
\omega=\left(0.6 \mathrm{M} / \mathrm{M}^{\prime}\right)^{1 / 2} \omega_{\mathrm{D}}
$$


where ( $\left.M / M^{\prime}\right)$ is the host-particle mass ratio, assumed to be large, and $\omega_{0}$ is the Debye frequency. This formula accounts for hydrogen mode frequencies in metal-hydrogen systems apart from those using palladium, which display other slightly unusual properties, (see section 6.4).

Detailed numerical calculations of the scattering response for a particle embedded in a matrix show that it contains a myriad of features [9]. For the two extreme cases of $Q \rightarrow 0$ and $Q \rightarrow \infty$ the response is well approximated by the fundamental contributions and a Gaussian envelope function, respectively. But, for intermediate $Q$ the line shapes are highly structured. Satellites to the harmonics appear which can be traced back to structure in the host density of states. Such features are understood by viewing the light mass particle as a probe of host lattice vibrations.

We turn now to scattering from collective atomic motions, or phonons. Neutron scattering is the established method of measuring phonon dispersion curves. To understand how this is possible consider the fundarnerital contribution to the harmonic oscillator response given in eqn. (16); this vanishes unless the neutron energy change matches the energy of one quantum. When scattering from a collective motion the energy selection-rule is supplemented by a wave vector selection-rule, $\mathbf{Q}=\mathbf{q}+\tau$ where $\mathbf{q}$ is the lattice wave vector (confined to a Brillouin zone) which labels the phonon mode. In an experiment both $Q$ and $\omega$ are determined, and hence a point on the phonon dispersion curve is established.

The one-phonon scheme we have just described has no value in tackling the interpretation of coherent scattering from a liquid for which two extreme limits are well understood. For small $Q$ and $\omega$ we can appeal to linear hydrodynamics. The response for fixed $Q$ is found to consist of an elastic peak (Rayleigh line) and two inelastic peaks (Brillouin lines) at $\omega= \pm Q c_{0}$ where $c_{0}$ is the velocity of sound. In the opposite limit of large $Q$ the response resembles that of a free particle, namely a Guassian centred at the recoil energy $E_{R}=(\hbar Q)^{2} / 2 M$ with a mean-square width proportional to $\left(K_{B} T E_{R}\right)$. While much has been learnt about the nature of physical processes engaged at intermediate $Q$, the subject is on-going, particularly with regard to molecular liquids and the super-cooled state. 


\subsection{Magnetic Scattering}

Neutrons scatter off the magnetic field generated by unpaired electrons in a sample. Note that the number of electrons involved is generally a small fraction of those engaged in an $x$-ray experiment.

The magnetic field has two sources. First, the spins of electrons lead to a dipole-dipole interaction. Second, mobile electrons generate a field obtained from Biot-Savart's formula. In the forward direction $(Q \rightarrow 0)$ the neutron-electron interaction is simply related to the total magnetic moment. Hence, for an atom characterized by total spin and angular momentum $\mathbf{S}$ and $\mathbf{L}$, respectively, the forward scattering amplitude is generated by $(\mathbf{L}+2 \mathbf{S})$.

The general form of the neutron-electron interaction for arbitrary $\mathbf{Q}$ is quite complicated. Calculation of the matrix elements required in the interpretation of neutron-electron spectroscopy of isolated ions requires the full battery of Racah algebra used in atomic and nuclear spectroscopy. Even so, we can gain a good insight into the behaviour of magnetic neutron scattering by employing a relatively simple expression for the interaction, valid for modest values of $Q$.

For not too large $Q$, the magnetic interaction operator is

$$
r_{0}(1 / 2) g F(Q) J
$$

where the interaction strength $r_{0}=-0.54 .10^{-12} \mathrm{~cm}, \mathrm{gJ}$ is the magnetic moment operator and $F(Q)$ is an atomic form factor. For a spin-only ion $g \mathbf{J} \rightarrow 2 S$, whereas for a rare earth ion $J=L+S$ and $g$ is the Lande' splitting factor. The form factor is defined to be unity in the forward direction, and decreases monotonically to a value of $\sim 0.2$ at $Q \sim 8$ $\AA^{-1}$ in a typical case. But, be warned, some form factors achieve a maximum value at a finite $Q[10]$.

The definitions of Bragg and total scattering carry over from the previous discussion of nuclear scattering. We begin our discussion with the total scattering from $\mathrm{N}$ paramagnetic ions, namely, 


$$
\frac{d \sigma}{d \Omega}=N\left[\left(r_{0} / 2\right) g F(Q)\right]^{2}(2 / 3) J(J+1)
$$

where $J$ is the magnitude of the spin, i.e. $J \cdot J=J(J+1)$. This formula shows that the cross-section is large for large values of $\mathrm{J}$, just as we would expect. The dependence on the scattering vector $Q$ comes only through the form factor $F(Q)$.

There is, of course, a very strong $Q$ dependence in Bragg scattering from an ordered magnetic material. First, scattering vanishes unless $Q=\tau$ where $\{\tau\}$ are reciprocal lattice vectors for the magnetic structure. A second dependence arises from the fact that the component of $\mathrm{J}$ perpendicular to $Q$ is observed. This feature often enables the moment orientation to be established. No such factor is explicit in (18) because the paramagnetic ions are randomly orientated. The Bragg cross-section for a collinear magnetic structure is

$$
\underset{\left(\frac{d \sigma}{d \Omega}\right)_{\text {coh }}^{\text {el }}}{=r_{0}^{2} N(2 \pi)^{3} / v_{0}} \sum_{\tau} \delta(Q-\tau) e^{-2 W(\tau)}\left|F_{M}(\tau)\right|^{2}\left[1-(\hat{\tau} \cdot \hat{\eta})^{2}\right]
$$

where $\hat{\eta}$ is a unit vector that defines the preferred magnetic (easy) axis and

$$
F_{M}(Q)=\sum_{d}(1 / 2) g_{d}<J>F_{d}(Q) \xi_{d} e^{10 \cdot d}
$$

in which $\xi_{d}= \pm 1$ according to the orientation of the magnetic moment relative to the preferred axis. Several features merit explicit mention. The total Bragg scattering from a magnetic material is described by the sum of (7) and (19). The moment, proportional to the thermal average $\langle J\rangle$, vanishes at the phase transition to the paramagnetic state. Bragg intensities can be used to obtain the critical exponent for the continuous decrease of the magnetization in the immediate vicinity of the transition. A magnetic material usually forms domains, in which case the orientation factor $\left\{1-(\hat{\tau} \cdot \hat{\eta})^{2}\right\}$ must be averaged over the easy axes for $\eta$. If all directions in space were equally likely, then clearly $(\hat{\tau} \hat{\eta})^{2}$ would average to $(1 / 3)$. The same result is also correct for cubic symmetry.

Diffuse magnetic scatteririg is observed with a mixed magnetic system. If one component of a binary system occurs with a small concentration then the diffuse crosssection can be shown to be proportional to the square of the spatial Fourier transform of the magnetization defect created in the host matrix. Analysis of measurements on 
such systems has been central to the development of the theory of the electronic structure of magnetic alloys. As the temperature of the alloy is raised toward the critical temperature, the spatial range of the defect increases. In consequence, the diffuse cross-section as a function of $Q$ becomes increasingly narrow, and it is believed to be singular at the critical temperature.

Next we discuss the elementary properties of neutron-electron spectroscopy. To be concrete consider the matrix elements for transistions between J-multiplets in the energy-level diagram of a rare earth ion. The ion can be regarded as isolated, to a good approximation, and not subject to a significant molecular field, for example. The expression (17) is no longer valid since it is based on the relation $L+2 S=g J$ which is restricted to a J-multiplet.

The matrix element <JM $|L+2 S| J^{\prime} M^{\prime}>$ vanishes except for $|J-J '|=1$; thus for small $Q$ we observe dipole-allowed transitions [10]. Beyond the limit of small $\mathbf{Q}$ higher-order transitions contribute to the cross-section. Even though the higher-order transitions are very weak compared to dipole-allowed contributions they have been unambiguously observed [12]. It is found that the energy separation between multiplets is essentially the same in concentrated and dilute magnetic systems if the levels come from a single Coulomb term. Significant differences are seen in data for the two types of system for levels that belong to different terms. This feature is attributed to screening of the Coulomb interaction by conduction electrons.

Data on dilute systems is readily obtained by optical spectroscopy. This technique is constrained by the dipole-selection rule, and it is not useful for concentrated metallic magnets. At present, neutron-electron spectroscopy, which is free of both constraints, has been successfully applied to transitions with energy separations up to $1.8 \mathrm{eV}$, and the technique is very much in its infancy.

Another form of magnetic spectroscopy is the study of crystal field levels. This is now very well established as a tool for direct observation of the crystal field energy level scheme [13], in $3 d$, rare earth and actinide compounds.

The introduction of exchange interactions between ions couples the single-ion crystal 
field states discussed in the preceding paragraph. Collective excitations are formed from phase-related linear combinations of the single-ion transitions. These exciton states, as they are usually called, display significant dispersion which can be followed throughout the Brillouin zone in many cases. The limiting factor is energy, but this has been ameliorated with the development of advanced pulsed sources and appropriate time-of-flight instrumentation.

A spin wave is the coherent propagation of a single unit of spin deviation. It is in some senses the magnetic analogue of a phonon, in as much that the neutron cross-section vanishes unless there is simultaneous conservation of energy and wave vector. Spin wave and phonon excitations can usually be distiriguished in the scattered spectrum by one of several simple tests. First, spin wave intensities decrease with increasing $Q$ because of the atomic form factor, whereas phonon intensities increase, with a $Q^{2}$ dependence. Secondly, a spin wave is an excitation away from an ordered state so it vanishes above the critical temperature. Hence, spin wave excitations are usually more sensitive than phonons to variations in ternperature. On approaching the transition temperature spin waves soften and become more heavily damped.

Neutron polarization analysis affords a completely unambiguous method by which to identify spin waves [7] because creation or annihilation of a spin wave induces polarization in an unpolarized neutron beam. Phonons do not produce such an effect, as might be expected from physical intuition.

Polarization analysis is even more useful when it comes to isolating paramagnetic spin fluctuation scattering, particularly when it is a case of studying a material with a high transition temperature and hence a strong phonon background. In the case of a paramagnet, which does not possess a preferred axis, there is no creation of polarization. An initially polarized beam has a final polarization in the direction of $\mathbf{Q}$, and a magnitude controlled by the projection of the incident polarization onto $\mathbf{Q}$. Hence, the final polarization vanishes if the incident polarization and $\mathbf{Q}$ are perpendicular, and it achieves a maximum value when they are parallel.

The main problem in practice has been to obtain efficient methods of producing polarized beams or of analysing the polarization of a beam scattered by the sample. 
Inelastic scattering events are typically $10^{-3}$ of the elastic intensity, so a relatively inefficient polarizing method that is tolerable for elastic studies may render inelastic measurements impossible.

At the time of writing there is renewed interest in the dynamics of paramagnetic spin fluctuations. Various experimental groups have reported, over the past few years, careful measurements of the paramagnetic response of insulating (EuO, EuS) and metallic ( $\mathrm{Fe}, \mathrm{Ni}, \mathrm{Pd}_{2} \mathrm{MnSn}$ ) systems. The consensus opinion is that the response evolves with increasing $Q$ from a Lorentzian-like function centred at $\omega=0$ to a squarer or top-hat function. But recent data for $\mathrm{Gd}$ [14] shows a distinct peak at finite $\omega$ for large $Q$, near the zone boundary, with a strong dispersion. This feature persists deep into the paramagnetic region, and there is, of now, no satisfactory explanation of this sloppyspin-wave-like excitation. 


\section{Illustrative Examples}

The use of neutron scattering in condensed matter and materials research began in real earriest in the early part of the nineteen fifties with particularly significant contributions coming from the USA and Canada. Instrumentational concepts from this time survived although now vastly more efficient because of fast dedicated computers and the benefits of a steady evolution in the efficiency of collimators, monochromators and detectors.

Luminaries in the subject gathered in Stockholm in October 1957 for a meeting on the use of slow neutrons to investigate the solid state: the organizers, Pauli and Stedman, attracted Brockhouse, Egelstaff, de Gennes, Larsson, Palevsky, Riste and Waller, among others, as key speakers. Several experiments on solids and liquids, irıcluding the first observation of the roton minimum in the superfluid $\mathrm{He}^{4}$ phonon dispersion curve, were made at the $\mathrm{R} 1$ reactor in Stockholm (see fig. 1). New possibilities came with the commissioning of the $\mathrm{R} 2$ reactor at Studsvik in 1962, and routine operation of state of the art diffractometers and spectrometers. Flux at the sample on a Studsvik instrument was significantly higher than on a comparable instrument placed at the heavy water reactor R1 in Stockholm. Ph.D. theses submitted to the universities of Gothenburg, Stockholm and Uppsala in the period 1968-1974 by, amongst others, Nelin, Nilsson, Sköld and Stedman contained results which attracted much acciaim. Stedman's contributions both in original instrument design (double crystal monochromator and constant-q method in phonon spectroscopy) and very precise lattice dynamics studies, mapping Fermi surface features observed in Kohn anomalies, stand out. In fig. 2 the experimental achievement during this period at Studsvik is demonstrated. Significant theoretical work was published somewhat ahead of the experimental programme by Sjölander; his review article published in 1965 [19] stimulated research on liquids and solids. In the dawn of Sweden's neutron research programme scientific interests did not include magnetic phenornena and this situation largely prevails today.

The following subsections are written with the aim of imparting to the reader a flavour of scientific progress in a range of topics that have benefited from use of the neutron scattering technique. At the sarne time, we give a perspective on work by Swedish scientists, using instrumentation based at the Studsvik reactor and overseas facilities. 
An effort has been made to reference recent papers and review articles.

Unfortunately, any choice of topics runs the risk of being invidious. To those researchers who do not find their work cited we hope no lasting offence is taken because surely none is intended. There is no special order in which topics are presented. With such a wealth of material to draw from we recognize that other authors could select a quite different set of topics and papers and still fulfil the same aims.

\subsection{Collective excitations in quantum fluids}

Helium-3 has such a high absorption for low energy neutrons that it is a favoured material for detectors. On the other hand, it forms at low temperatures an almost perfect quantum Fermi liquid which is of interest in its own right and as a guide to other, more complicated, Fermi systems. Among these are electrons in metals, where there is the added complication of scattering by ion cores, nuclei which are finite size twocomponent systems, and neutron stars whose remoteness make them less than ideal subjects for microscopic studies.

One intriguing phenomenon in liquid $\mathrm{He}^{3}$ is the existence of a collective density oscillation which, in some respects, resembles a sound mode. However, as made clear by Landau in his seminal papers published in 1956, zero-sound is a purely quantum phenomenon, associated with distortions in the Fermi surface, which occurs in the collisionless regime of dynamics. Ordinary sound is a purely classical phenomenon which occurs in the hydrodynamic (collision dominated) regime of many particle dynamics.

The dispersion of zero-sound, obtained with inelastic neutron scattering, was first reported by Sköld et al. [20] (fig. 3). These experimentalists surmounted the seemingly daunting problem due to absorption by using a clever design for the sample container and counting for very long times (at a medium flux reactor) with a correlation chopper spectrometer. Since then the damping and pressure dependence of zero-sound has been measured [21]. Mixtures of the quantum liquids $\mathrm{He}^{3}$ and $\mathrm{He}^{4}$ (Fermi and Bose liquids, respectively) pose challenges to theoretical physicists. While existing theories 
are moderately successful when confronted with data [22] a number of iriteresting features remain unexplained.

\subsection{Are classical liquids hard sphere assemblies?}

By and large, the structure of a monatomic liquid is the same as that of an assembly of hard spheres of the appropriate radius and packing density. This is the conclusion of $x$-ray and neutron diffraction measurements of the static structure factor $S(Q)$ defined in eqn (11). Neutron diffraction data for the structure factor of liquid lead are shown in fig. 4 together with the appropriate hard-sphere values. One can thus conclude that the physically essential information obtainable from a $S(Q)$ is to be found in the relatively small difference between the measured curve and the corresponding hard-sphere quantity.

This is a sad state of affairs, since it means that it is a struggle to extract information on the molecular potential from measurements of $S(Q)$. It also means that measurements and data analysis must be performed with great attention to detail if useful results are to be obtained. Both have been refined to a state where molecular potentials are extracted through an iterative process in which computer simulation and neutron scattering results are compared.

Computer simulations and neutron scattering experiments go hand-in-hand. There is probably no better example of the impact of the twin approach than in the advancements made in the past few years in understanding the static and dynamic properties of liquids [23]. It is fair to say that up to quite recently simulation studies set a standard for neutron measurements, but now groups in Sweden (Dahlborg and collaborators) and the Netherlands (de Graaf and collaborators) have brought the accuracy of neutron measurements on a par with highest quality simulation data. It is even possible to go as far as obtaining from data correlation functions in space and time which are the natural co-ordinates in a computer simulation $[24,25]$.

Results for bismuth, a semi-metal, illustrate what can be achieved. First, a molecular poteritial has been constructed which yields simulation data in accord with measured 
values of $S(Q)$ (see fig. 5), including a shoulder on the large $Q$ side of the main peak in the structure factor that seems to be a characteristic feature of semi-metals [26] (and not present in $S(Q)$ for a hard-sphere assembly shown in fig. 4). Secondly, the selfparticle dynamics has been extracted from the data, even though bismuth is a totally coherent scatterer [25]. The data have then been analysed in terms of the mean-square displacement which, as expected from simple physical considerations, increases linearly with time at sufficiently long times with a gradient proportional to the diffusion constant. Values of the latter obtained from computer simulations and scattering experiments agree well with the observed value (fig.6). This is the first time this has been demonstrated. At intermediate times, before the diffusive region is reached, there is a significant discrepancy between simulation and neutron scattering results, which awaits a satisfactory explanation.

\subsection{Structure and dynamics in hydrogenous substances}

Water, ever present in living matter, and other hydrogen bonded systems have been extensively investigated with $x$-ray and neutron scattering by several groups at almost all universities in Sweden. The amount of effort devoted to such systems reflects both their basic importance in chemistry and biology, and the very convenient neutron scattering properties of the proton and deuteron which allows use of the isotope substitutional technique. In this report we will not consider liquid water (although it has been studied extensively during the 60 's) and we refer the interested reader to a review article [27].

Protons are strong incoherent neutron scatterers, so the signal from a hydrogenous material is dominated by the properties of individual protons, whereas deuterons are predominantly coherent scatterers. Bragg scattering from deuterated crystals thus yields useful structural information whereas $X$-ray scattering is poor in locating protons because of the weak scattering power of hydrogen.

In conjunction with vibrational and NMR spectroscopy and theoretical calculations neutron and $x$-ray diffraction have been key tools in a long-standing hydrogen bond project co-ordinated by Olovsson at Uppsala University. This project has focussed on a 
broad range of structural and dynamical molecular-level aspects of the chemistry and physics of $\mathrm{H}$-bonded crystals. Neutron diffraction became early an essential technique in many of the problem areas studied by this group, such as structure and dynamics of water in solids [28], electron density mappings [29], the hydrated proton (ref. [30] and references therein), ferroelectricity and other physical properties [31], $\mathrm{H}$-bond geometry and models for $\mathrm{H}$-bond interactions [32], order-disorder problems in $\mathrm{H}$-bonds [33] and proton conductors [34].

A recent example of work in this project is the determination of the electron density in $\mathrm{NaHC}_{2} \mathrm{O}_{4} \cdot \mathrm{H}_{2} \mathrm{O}$ at $120 \mathrm{~K}$, using $\mathrm{x}$-ray and neutron diffraction techniques [35]. This is one of a limited number of investigations at low temperatures of the effect of the crystalline field on the charge density of the water molecule (see fig. 7). Deformation densities in the three independent hydrogen bonds are consistent with an electrostatic model for bonds of weak to intermediate strength in which polarization and other contributions become more important as the proton-acceptor distance decreases. The hydrogen bonding affects both the covalent bonds and the lone-pair electron density of the acceptor oxygen atoms.

Another example of the function of $\mathrm{H}$-atoms in crystals is the fact that the presence of hydrogen can induce structural ordering, although the mechanism for this is not always well understood. The $\mathrm{Pd}_{3} \mathrm{Mn}$ structure for example crystallizes in a disordered $\mathrm{fcc}$ structure above approximately $800 \mathrm{~K}$, and sluggishly transforms to an ordered tetragonal structure at lower temperatures. In the presence of hydrogen the initially disordered sample transforms to an ordered structure. As a first step toward understanding the mechanism of this type of ordering, the locations of the hydrogen atoms were determined by powder diffraction using a Rietveld profile refinement technique [36]. It was found that the deuterium atoms mainly occupy octahedral sites with six nearest-neighbour Pd sites (fig. 8).

Today powder diffraction methods in conjunction with the successful implementation of the Rietveld refinement technique scheme [37] has become a very useful structuresolving tool to the extent that it competes favourably with single-crystal work. 
6.4 Proton jumps and vibrations in hydrogen-metal systems.

Research on hydrogen-metal systems forms a small industry of work by chemists, physicists and metallurgists pursuing strategic and basic goals. Strategic work is aimed at energy storage, fusion technology, hydrogen embrittlement and the like. In addition, due largely to the small mass of the hydrogen, there are a variety of subtle effects such as fast hydrogen diffusion, large isotope effects, and hydrogen tunnelling. Moreover, there is a conceptual relation between hydrogen and muon behaviour in metals [38].

At low concentrations, hydrogen atoms undergo a diffusive motion when viewed over long (macroscopic) times. The standard model assumes that diffusion occurs over welllocalized interstitial sites interconnected by a set of jump vectors $\mathbf{e}$. The jump probability to any of the $n$ adjacent sites is $(1 / n \tau)$ where $\tau$ is the residence time at a given site. For a simple Bravais interstitial lattice the cross-section is a Lorentzian like eqn (14) with a half-width at half-height,

$\Gamma(Q)=(1 / n \tau) \sum_{j=1}^{n}\left[1-\cos \left(Q e_{j}\right)\right]\left\{=Q^{2} Q^{2} / \tau ; Q \rightarrow 0\right\}$

where the second equality defines a length $\ell$ introduced in section 5.1.3.

The first experiment in which the quasielastic scattering from a metal hydride was interpreted in terms of diffusion was that by Sköld and Nelin [39] on hydrogen in fcc palladium ( $\alpha$-phase) in 1967 (see fig. 9). Bcc metals are more complicated to model. At room temperature the standard model works quite well, but at higher temperatures the measured width is significantly smaller than calculated values. Satisfactory agreement between measurements and theory is achieved when the latter is generalized to include a double-jump mechanism with sequences of two rapid jumps to nearest and next-nearest neighbor interstitial positions and also backward jumps.

Additional information can be extracted from measurements by analysing the intensity of the quasielastic line. In this respect, eqn (14) is modified by inclusion of a DebyeWaller factor $\exp \left(-Q^{2}\left\langle u^{2}\right\rangle\right)$ where $\left\langle u^{2}\right\rangle$ is interpreted as the mean-square vibration amplitude of the hydrogen during its residence time. Values of $\left\langle u^{2}>\right.$ vary between 0.02-0.08 $\AA^{2}$ depending on the system and temperature. The value for hydrogen in palladium is particularly large, and this is attributed to the relatively low frequency of the 
localized mode in this system, mentioned already in section 5.1.3.

When viewed over short times a hydrogen atom vibrates about an interstitial site. This can be viewed as motion of a particle in a harmonic potential, centred at the interstitial site, to a good approximation. Better still, it is modelled by an impurity embedded in a matrix of atoms, as mentioned in section 5.1.3. The latter approach stresses the picture of a hydrogen atom as a probe of lattice dynamics, in much the same way as is customary when interpreting rnuon behaviour in a metal. Experiments on localized modes are very much improved by using a spallation source because they occur at energies around $130 \mathrm{meV}$ at which the intensity and resolution available at reactor based spectrometers has seriously degraded $[40,41]$.

\subsection{Zero-momerit magnetic materials}

Magnets that contain rare earth or actinide ions (4f and $5 f$ unpaired electrons, respectively) possess a rich variety of features which can border on exotic. Rare earth magnets are relatively easy to understand because $4 f$ electrons are compact and hence not too strongly perturbed by the local environment and conduction electrons. Nonetheless, some properties, of even elemental rare earth magnets such as gadolinium, defy explanation with current theoretical ideas and methods [14,42].

But here we want to draw attention to an intriguing effect in some actinide magnets, namely, the almost complete cancellation of spin and orbital contributions which results in a zero total moment. Johansson and colleagues [43] attribute this to strong interactions between the $f$ and conduction electrons ( $s, p$ and $d$ ) aiding the interatomic correlations which produce oppositely aligned spin and orbital moments in the first half of $f$ electron series. They predict that $\mathrm{UFe}_{2}$ is an intinerant ferromagnet with a magnetic moment due mainly to the iron moments. From the discussion given in section 5.2 we conclude that the magnetic neutron scattering amplitude for the uranium ion should be very small near the forward scattering direction.

Wulff et al. [9] have confirmed this to be the case. The measured total uranium momerit is zero within experimental error. The $\mathrm{Q}$-dependence of the corresponding magnetic 
amplitude is very strong and in accord with theoretical predictions by Brooks et al [43].

\subsection{Arresting Relaxation in Liquids}

Our understanding of glasses is very much in its infancy. With silica glass, for example, it is commonly believed that the structure should contain $\mathrm{SiO}_{4}$ tetrahedra similar to those of the different forms of crystalline $\mathrm{SiO}_{2}$ [44]. The nature of short and medium range order is a subject of much current debate. None of the existing models is in complete accord with $\mathrm{x}$-ray and neutron diffraction work. Nor is there satisfactory agreement with available computer simulations. As with studies of liquids, there is a need for accurate data for the static structure factor $S(Q)$ and dynamic scattering function $S(Q, \omega)$ over wide ranges of $Q$ and $\omega$.

A new kind of intermediate range ordering has recently been observed in superionic borate, phosphate and molybdate glasses. It manifests itself in an anomalous peak in $S(Q)$ at a low $Q$ of $0.8 \AA^{-1}[45]$ (fig. 10). Torell and collaborators attribute this to the formation and ordering of Agl-clusters. There are strong indications from neutron diffraction in combination with a variety of other experimental results that the Agl clusters constitute a fractal percolating network within the host glass which serves as a conducting pathway for the $\mathrm{Ag}^{+}$ions $[46,47]$.

A theory of the glass transition developed by Sjölander and collaborators [48-52] is based on the premise that the transition is basically of a dynamic nature, which stems from changes in the self-diffusion constant and viscosity, rather than driven by a mode softening at the homogeneous nucleation limit. In a supercooled liquid structural relaxation times, governed by atomic diffusion, are very long. Ultimately, a glass state is formed in which atoms form a disordered solid, structural degrees of freedom are frozen out, and structural relaxation times are truly macroscopic. At an atomic level, motion of a single tagged particle involves a non-linear coupling of self- and collective motions which gives rise to a cage effect where the medium blocks the particle's passage. The cage effect progresses to self-trapping in the super-cooled state, due to the slow response of the liquid to disturbances, and this results in a small value of the self-diffusion constant. The theory focuses on the normalized intermediate scattering function which is postulated to have a particular functional shape. 
These concepts have largely been vindicated by computer simulation [53-55] and neutron scattering studies [56-58]. In particular, there is good evidence for the predicted two slow-relaxation steps, i.e. slow on the time scale of normal lattice vibrations (see fig. 11). One of the two steps exists on both sides of the transition temperature and slows down critically. The second step exists only in the liquid phase, and when the transition is approached the divergence of the associated time scale outstrips the time scale for the first step. There is also a divergence of transport constants, as might be expected. However, since the transition is not a continuous phase transition, as in a simple magnet, established universality classes and scaling concepts do not apply. Even so, theory predicts that the second relaxation process obeys a master curve, which is a form of scaling, and this feature is indeed borne out by experiment.

\section{Future trends}

The few examples discussed above just give a flavour of the overwhelming possibilities of the neutron scattering technique. Many areas were not even mentioned such as the small-angle scattering technique by which important contributions to many different kinds of sciences has been made. On the one hand, the neutron scattering technique complements other experimental methods, like $\mathrm{X}$-ray and light scattering, but in many instances it provides information not available by other means. It has also the advantage of being a direct probe which makes meaningful comparisons between theoretical predictions and experimental results feasible provided, of course, that the experimental data has been treated with up-to-date methods with respect to data correction procedures.

What are the general trends and how will the technique develop in the future?

Several different research areas of increased interest can be identified. To be mentioned here is especially the enhanced use of polarized neutrons which has been made possible through the development of efficient polarizers, above all the supermirror [7]. This has resulted in an efficient tool that makes it easy to distinguish between nuclear and magnetic phenomena in a scattering experiment and this can now be used with advantage also at reactors of medium source strength. The construction of a facility for 
polarized neutrons at the $\mathrm{R} 2$ reactor is in line with this development and it is anticipated that it will be of great use for Swedish scientists in the future. Other developments which also certainly will continue is the increased interest in studies of complex chemical systems, amorphous materials (both magnetic and non-magnetic), thin films, biological substances, the energy levels of the crystal field in solids, etc.

Another insight which has slowly emanated over the years among neutron scattering scientists is the observation that one experimental technique alone is not able to give answers to all questions. This does also apply to the neutron scattering technique and thus a stronger and stronger interplay between neutron scattering and other experimental methods (X-ray diffraction, light scattering and absorption, molecular dynamics simulations) have to be employed in order to map static and dynamic phenomena especially in complex systems. Complex in this respect means both systems which behave in a complicated and subtle way, but also chemically complex. As an example we can mention ordinary water, the behaviour and interaction of which on a molecular level, even after intense scientific onslaught over many decades, is to a large degree unexplained, materials of technological interest like soft amorphous and partly crystalized magnetic materials.

In order to extract some relevant information today from a diffraction or from a neutron inelastic scattering experiment it is necessary not only to have high quality data (good statistics, large enough Q- $\omega$ range, appropriate resolution in order to unravel the desired information from the measured data, etc) but also to have as a full control of the experiment itself as possible. What is even more important is that the experimentalist must be able to accurately correct the data for all the experimental effects. These criteria work hand in hand and to improve one and not the others to the same degree is meaningless. For example, with the advent of dedicated spectrometers for measurements of dynamic scattering functions $S(Q, \omega)$ at ILL, ISIS, and Studsvik it is thus essential to put a lot of effort into the development of accurate correction procedures. In order to optimize the experimental accuracy it will be more and more common to simulate the complete experiment on a computer by Monte-Carlo methods before it is actually performed.

It is definitely so that the pulsed neutron sources, especially ISIS, have brought a new dimension into the neutron scattering field and that they will be of outstanding 
importance (two examples only to be mentioned) for studies of magnetic phenomena and for studies of high-energy collective excitations at small $Q$. It should however be pointed out that a high-flux research reactor like the one at ILL and the ISIS spallation source are complementary. Both kinds of sources are needed in order to cover in the best possible way the vast research areas where the neutron scattering technique can give unique and substantial information about microscopic phenomena. It has also to be observed that this does not mean that the scientific case for medium flux research reactors, like $R 2$, is defunct. There are many examples where the quality of the data obtained at for example R2 or other smaller research reactors are as good as those obtained at the ILL. We should not dwell into explaining the reasons for this but rather state it as a fact. It is certainly also so that the exploratory measurements in the future preferably are to be made at smaller centres as well as those experiments requiring very complicated and fancy auxiliary equipment. 


\section{Acknowledgements}

We are grateful to K. Hermansson, E.B. Karlsson, I. Olovsson, K. Sköld, R. Tellgren, J.O. Thomas and L.M. Torell for discussions and correspondence. 


\section{References}

1. Scherm, R.H. in The Art of Measurernent, edited by B. Kramer (VCH, Weinheim 1988)

2. Lovesey S.W. Theory of Neutron Scattering from Condensed Matter, Vols 1 and 2, 3rd impression (Oxford University Press, Oxford, 1987)

3. Neutron Scattering, Methods of Experimental Physics Vols 23A,B,C, edited by D.L. Price and K. Sköld (Academic Press, N.Y. 1986)

4. Neutron Scattering at a Pulsed Source, edited by R.J. Newport, B.D. Rainford and R. Cywinski (Adam Hilger, Bristol, 1988)

5. Lovesey, S. W., Inst. Phys. Conf. Ser. No 101, 133 (1990).

6. Moon, R.M. and West, C.D., Physica 137B, 347 (1986)

7. Williams, W.G., Polarized Neutrons (Oxford University Press, Oxford, 1988).

8. Bee, M., Quasi-elastic Neutron Scattering (Adam Hilger, 1989)

9. Williams, R.D. and Lovesey, S.W., Z. Phys. B62, 413 (1986)

10. Wulff, M., Lander, G. H., Lebech, B. and Delapalme, A., Phys Rev B39, 4719 (1989)

11. Williams, W.G., Boland, B.C., Bowden, Z.A., Taylor, A.D., Culverhouse, S. and Rainford, B.D., J. Phys. F17, L151 (1987)

12. Taylor, A.D., Osborn, R., McEwen, K.A., Stirling, W.G., Bowden, Z.A., Balcar, E. and Lovesey, S.W., Phys. Rev. Lett. 61, 1309 (1988). 
13. Fulde, P. and Loewenhaupt, M., Adv. Phys. 34, 589 (1986).

14. Cable, J.W., and Nicklow, R.M., Phys. Rev. B39, 11732 (1989).

15. Palevsky, H., Otnes, K. and Larsson, K.-E., Phys. Rev. 112, 11 (1958)

16. Brockhouse, B.N. and Stewart, A.T., Rev. Mod. Phys. 30, 236 (1958)

17. Larsson, K.-E., Dahlborg, U. and Holmryd, S., Ark. Fys. 17, 369 (1960)

18. Stedman, R. and Nilsson, G., Phys. Rev. 145, 492 (1966)

19. Sjölander, A., chap. 7 in Thermal Neutron Scattering, edited by P.A.Egelstaff, Academic Press, London and New York, 1965

20. Sköld, K., Pelizzari, C.A., Kleb, R. and Ostrowski, G., Phys. Rev. Letters 37, 842 (1976)

21. Scherm, R., Guckelsberger, K., Fak, B., Sköld, K., Dianoux, A. J., Godfrin, H. and Stirling, W.G., Phys. Rev. Lett. 59, 217 (1987).

22. Scherm, R., Dianoux, A.J., Fak, B., Guckelsberger, K., Körfer, M and Stirling, W.G., Physica B 156 and 157, 311 (1989).

23. Larsson, K-E., Phys. Chem. Liq. 12, 273 (1983)

24. Dahlborg, U., Söderström, O., Gudowski, W., Larsson, K.-E. and Davidovic, M., J .Phys. F: Met. Phys. 15, 2053 (1985)

25. Dahlborg U, Gudowski, W. and Davidovic, M., J. Condens. Matter 1, 6173 (1989).

26. Dzugutov, M. and Dahlborg, U., Phys. Rev. A40, 4103 (1989).

27. Hermansson, K., Portugaliae Physica 19, 133 (1988). 
28. Eriksson, A. and Hermansson, K., Acta Cryst. B 39, 703 (1983)

29. Hermansson, K., Acta Cryst. B 41, 161 (1985)

30. Gustafsson, T., Acta Cryst. C 43, 816 (1987)

31. Liminga, R. and Tellgren, R., Acta cryst. B 38, 1551 (1982)

32. Olovsson, I. and Jönsson, P.G., chap. 8 in The hydrogen bond - recent developments in theory and experiments, edited by P.Schuster et al. (North-Holland Publ. Co, Amsterdam) 1976.

33. Thomas, J.O., Tellgren, R. and Olovsson, I., Acta Cryst. B 30, 2540 (1974)

34. Thomas, J.O. and Farrington, G.C., Acta Cryst B 29, 227 (1983)

35. Delaplane, R.G., Tellgren, R. and Olovsson, I., Acta Cryst. B 46, 361 (1990)

36. Ahlzen, P.-J., Andersson, Y., Tellgren, R., Rodic, D., Flanagan, T.B. and Sakamoto, Y.Z., Z. Phys. Chem. 163, 213 (1989)

37. Rietveld, H.M., J. Appl. Phys. 2, 65 (1969)

38. Karlsson, E.B. Int. J. Quan. Chem. XXXV, 779 (1989)

39. Sköld, K. and Nelin, G., J. Phys. Chem. Solids 28, 2369 (1967)

40. Bennington, S.M., Benham, M.J., Ross, D.K., Taylor, A.D. and Bowden, Z.A., Z. Phys. Chem. 164, 1071 (1989)

41. Noreus, D. and Tomkinson, J., Chem. Phys. Lett. 154, 439 (1989) 
42. Hartmann, O., Karlsson, E.B., Wäppling, R., Chappert, J., Yaouanc, A., Asch, L., and Kalvius, G.M., J. Phys. F: Met Phys. 16, 1593 (1986).

43. Brooks; M.S.S., Eriksson, O., Johansson, B., Franse, J.J.M., and Frings, P.H., J. Phys F.18, L33 (1988).

44. Graneli, B. and Dahlborg, U., J. Non-Cryst. Solids 109, 295 (1989).

45. Börjesson, L., Torell, L.M., Dahlborg, U., and Howells, W.S., Phys. Rev. B 39, 3404 (1989)

46. Börjesson, L., Torell, L.M. and Howells, W.S., Phil. Mag. B 59, 105 (1989)

47. Börjesson, L. and Howells, W.S., Solid State lonics (in press).

48. Bengtzelius, U., Götze, W. and Sjölander, A., J. Phys. C 17, 5915 (1984)

49. Bengtzelius, U. and Sjögren, L., J. Chem. Phys. 85, 1744 (1986)

50. Götze, W., Z. Phys. B 60, 195 (1984)

51. Götze, W., Phys. Scripta 34, 66 (1986)

52. Götze, W. and Sjögren, L., Z. Phys. B 65, 415 (1987)

53. Signorini, G.F., Barrat, J-L., and Klein, M.L., J. Chem. Phys 92, 1294 (1990)

54. Kinell, T. and Lovesey, S.W., J. Phys. C 19, L791 (1986)

55. Dzugutov, M. and Dahlborg, U., Proc. from International Discussion Meeting on Relaxations in Complex Systems, Heraklion June 18-29, 1990. (to be published)

56. Mezei, F., Knaak, W. and Farago, B., Eur. Phys. Lett. 7, 529 (1988) 
.066 I IE

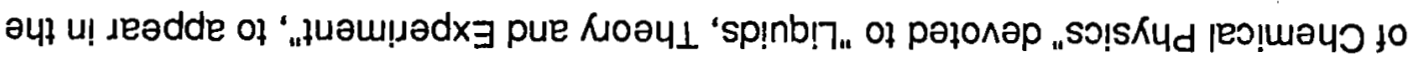

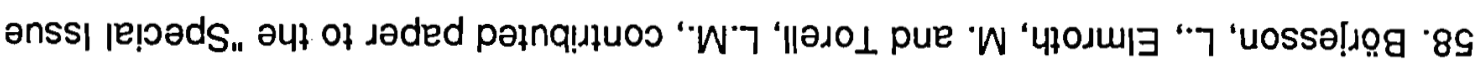

(686)

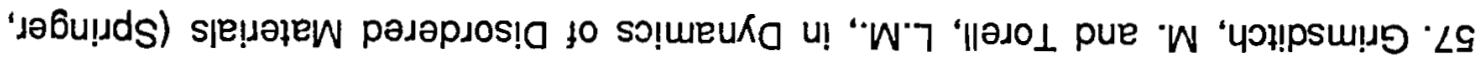
$8 \varepsilon$ 
Table 1.

Neutron scattering and absorption data for some selected elements (taken from a compilation by L.Koester and W.B.Yelon, 1982.)

\begin{tabular}{|c|c|c|c|c|c|}
\hline Element & $A$ & $\%$ abundance & $b$ & $\sigma_{\mathrm{s}}$ & $\sigma_{\mathrm{a}}$ \\
\hline \multirow[t]{3}{*}{$\mathrm{H}$} & 1 & 100 & -0.3741 & 81.87 & 0.3326 \\
\hline & 2 & 0.0149 & 0.6674 & 7.63 & 0.000519 \\
\hline & 3 & & 0.494 & 3.03 & $<0.000006$ \\
\hline \multirow[t]{3}{*}{$\mathrm{He}$} & $\star$ & & 0.326 & 1.21 & $<0.001$ \\
\hline & 3 & 0.00014 & 0.574 & 5.6 & 5333. \\
\hline & 4 & 100. & 0.326 & 1.21 & $\sim 0$ \\
\hline \multirow[t]{4}{*}{$\mathrm{Li}$} & $\star$ & & -0.203 & 1.40 & 70.5 \\
\hline & 6 & 7.5 & 0.187 & 0.98 & 940. \\
\hline & & & $-0.026 i$ & & \\
\hline & 7 & 92.5 & -0.220 & 1.44 & 0.0454 \\
\hline \multirow[t]{4}{*}{$\mathrm{C}$} & * & & 0.6648 & 5.564 & 0.00350 \\
\hline & 12 & 98.89 & 0.6653 & 5.564 & 0.00353 \\
\hline & 13 & 1.11 & 0.62 & 5.5 & 0.00137 \\
\hline & 14 & & & & $<10^{-6}$ \\
\hline \multirow[t]{4}{*}{0} & * & & 0.5805 & 4.234 & 0.00019 \\
\hline & 16 & 99.762 & 0.5805 & 4.234 & 0.00019 \\
\hline & 17 & 0.038 & 0.578 & 4.20 & 0.24 \\
\hline & 18 & 0.200 & 0.584 & 4.3 & 0.00016 \\
\hline $\mathrm{Na}$ & 23 & 100. & 0.363 & 3.23 & 0.530 \\
\hline \multirow[t]{3}{*}{$\mathrm{Cl}$} & * & & 0.9579 & 16.63 & 33.5 \\
\hline & 35 & 75.77 & 1.17 & 21.62 & 44.1 \\
\hline & 37 & 24.23 & 0.308 & 1.2 & 0.433 \\
\hline \multirow[t]{4}{*}{ K } & $\star$ & & 0.367 & 2.10 & 2.1 \\
\hline & 39 & 93.258 & 0.379 & 2.19 & 2.1 \\
\hline & 40 & 0.012 & & & 34. \\
\hline & 41 & 6.73 & 0.258 & 0.83 & 1.46 \\
\hline
\end{tabular}




\begin{tabular}{|c|c|c|c|c|c|}
\hline \multirow{3}{*}{ V } & * & & -0.0382 & 4.953 & 5.08 \\
\hline & 50 & 0.25 & & & 60. \\
\hline & 51 & 99.75 & -0.0414 & 4.946 & 4.9 \\
\hline \multirow[t]{5}{*}{$\mathrm{Fe}$} & $*$ & & 0.954 & 11.66 & 2.56 \\
\hline & 54 & 5.8 & 0.42 & 2.2 & 2.3 \\
\hline & 56 & 91.72 & 1.01 & 12.8 & 2.6 \\
\hline & 57 & 2.2 & 0.23 & $<1$ & 2.5 \\
\hline & 58 & 0.28 & 1.5 & 28. & 1.28 \\
\hline \multirow[t]{6}{*}{$\mathrm{Ni}$} & * & & 1.03 & 17.56 & 4.5 \\
\hline & 58 & 68.27 & 1.44 & 25.87 & 4.6 \\
\hline & 60 & 26.10 & 0.28 & 0.96 & 2.9 \\
\hline & 61 & 1.13 & 0.76 & 7.23 & 2.5 \\
\hline & 62 & 3.59 & -0.87 & 9.6 & 14.5 \\
\hline & 64 & 0.91 & -0.038 & 0.02 & 1.52 \\
\hline \multirow[t]{3}{*}{$\mathrm{Ag}$} & * & & 0.597 & 5.09 & 63.3 \\
\hline & 107 & 51.83 & 0.764 & & 38. \\
\hline & 109 & 48.17 & 0.419 & & 91. \\
\hline \multirow[t]{5}{*}{$\mathrm{Pb}$} & * & & 0.9401 & 11.11 & 0.17 \\
\hline & 204 & 1.4 & & & 0.66 \\
\hline & 206 & 24.1 & & & 0.0305 \\
\hline & 207 & 22.1 & & & 0.709 \\
\hline & 208 & 52.4 & & & 0.00049 \\
\hline $\mathrm{Bi}$ & 209 & 100. & 0.8533 & 9.156 & 0.033 \\
\hline
\end{tabular}

$\mathrm{b}\left(10^{-12} \mathrm{~cm}\right)$ Coherent scattering length for bound atoms. Complex values correspond to a neutron wave length of $1 \AA$.

$\sigma_{\mathrm{s}}$ (barns) Total scattering cross sections of bound atoms for thermal neutrons

$\sigma_{\mathrm{a}}$ (barns) Absorption cross sections for thermal neutrons ( $2200 \mathrm{~m} / \mathrm{sec}$ )

Natural isotope mixture 
Table 2.

Characteristic parameters of neutron-producing reactions used in neutron sources

$\begin{array}{lllll}\text { reaction } & \begin{array}{l}\text { number of } \\ \text { neutrons }\end{array} & \text { example } & \begin{array}{l}\text { neutron } \\ \text { production }\end{array} & \begin{array}{l}\text { target } \\ \text { power }\end{array} \\ \text { fission } & -2.5 \text { per } & \text { ILL high-flux } & 2 \times 10^{18} \mathrm{n} / \mathrm{s} & 57 \mathrm{MW} \\ & \text { fission } & \text { reactor } & & \\ & (1 \text { effective) } & \text { (continuous) } & & \end{array}$

spallation $\quad-26$ per $800 \quad$ RAL spallation $\quad 3 \times 10^{16} \mathrm{n} / \mathrm{s} \quad \sim 0.2 \mathrm{MW}$

in $U^{238} \quad$ MeV proton source (pulsed) 
Figure captions

Fig. 1. Dispersion curve for the excitations in superfluid ${ }^{4} \mathrm{He}$ at about 1.5K. The dashed line is the prediction of the Larıdau-Feynman theory. (from Palevsky et al [15])

Fig.2. Demonstration of the enormous increase in accuracy of the measured dispersion curves during the late 50's and early 60's. As example are results for aluminium in the [200] direction of the Brillouin zone taken. a) Brockhouse and Stewart 1958 [16], b) Larsson et al 1960 [17], c) Stedman and Nilsson 1966 [18]. The dashed and full lines correspond to different theories.

Fig. 3. a) Example fit of a phenomenological function to measured data. The dashed curves are the individual comporients of the fit (spin fluctuation, zero-sound and multi-pair) and the solid cirve is the sum. b) Dispersion relation for collective ("zero-sound") excitations in liquid ${ }^{3} \mathrm{He}$. Solid curves are theoretical predictions and dashed line an extrapolation of the zero-sound velocity at low frequencies. (Sköld, private communication)

Fig. 4. Measured $S(Q)$ for liquid lead (circles) and calculated curve according to the Percus-Yevick approximation for hard spheres.

Fig. 5. Comparison between the zeroth moments of the dynamic scattering function $S(Q)$ obtained from an optimized molecular dynamics simulation procedure [ ] and experimental results. The small $Q$ region is shown in the inset. (from Dzugutov and Dahlborg [26])

Fig. 6. The mean square displacement (MSD) of atom obtained from the width of the self correlation correlation function $G_{s}(r, t)$ for liquid bismuth. The full curve corresponds to molecular dynamics simulation results. (from Dahlborg et al [25]) 
Fig. 7. (a) The geometry of $\mathrm{NaHC}_{2} \mathrm{O}_{4}$ at $120 \mathrm{~K}$. Thermal ellipsoids are plotted at $75 \%$ probability. (b) to (d) show the experimental deformation densities in the $\mathrm{HC}_{2} \mathrm{O}_{4}$ ion. Each composite map has been plotted separately through the $O(1), C(1)$, $O(2)$ and $O(3), C(2), O(4)$ planes. (b) $X-N$ difference density, (c) high-order $X-$ $(X, N)$ difference density, (d) dynamic $(X, N)$ multipole deformation density. Contours are at intervals of $0.05 \mathrm{e}^{-3}$. Solid and dashed lines denote positive and negative contours, respectively; the zero-level has been omitted. (from Delaplane et al. [35])

Fig.8. The tetragonal structure of $\mathrm{Pd}_{3} \mathrm{Mn}$. The coordinations of the two deuterium positions are shown in the lower part of the figure. In the upper part, the stacking sequence is indicated. (from Ahlzen et al. [36])

Fig. 9. Observed width of the scattering function in $\mathrm{Pd}-\mathrm{H}$ for three temperatures. The curves predicted by the octahedral model are shown by the solid lines for the best value of $\tau$ at each temperature. Dashed line shows the curve predicted by the tetrahedral model for an arbitrary chosen value of $\tau$. (from Sköld and Nelin [39])

Fig. 10. The structure factors for two $(\mathrm{Agl})_{x}-\left(\mathrm{Ag}_{2} \mathrm{O}-2 \mathrm{~B}_{2} \mathrm{O}_{3}\right)_{1-x}$ glasses with $\mathrm{x}=0.6$ (upper curves) and $x=0.1$ (lower curves) measured on the liquid and amorphous diffractometer LAD at ISIS at scattering angles (a) $146^{\circ}$ and (b) $10^{\circ}$. The upper curves have been shifted by 0.3 units to higher values for clarity. The difference between the intensities of the $x=0.5$ (upper curve), the $x=0.3$ (lower curve) glass and the undoped binary glass $x=0$ is shown in (c) for the $10^{\circ}$ scattering angle. (from Börjesson et al [46])

Fig.11. The normalized density correlation function at the reduced temperature 0.5 . solid line: $Q \sigma=7$; dashed line: $Q \sigma=6.2$; chain-dotted line: $Q \sigma=5.4$. $\sigma$ is a characteristic length and $\tau$ is characteristic time related to the particular shape of the assumed interaction potential. The squares represent fits of Kohlrausch functions. The time scales of two different relaxation mechanisms are clearly seen. (from Dzugutov and Dahlborg [55]) 


\section{INSTRUMENTATION FOR THERMAL NEUTRON SCATTERING AT THE REACTOR R2, STUDSVIK}

\begin{tabular}{|c|c|c|}
\hline $\begin{array}{l}\text { Neutron } \\
\text { beam tube }\end{array}$ & Instrumentation & Responsible \\
\hline $\mathrm{H1}$ & Service instrument & $\begin{array}{l}\text { NFL } \\
\text { (scientist: W.Gudowski) }\end{array}$ \\
\hline H3 & $\begin{array}{l}\text { Time-of-flight spectrometer } \\
\text { for thermal neutrons } \\
\text { (operational summer 1990) }\end{array}$ & $\begin{array}{l}\text { Department of Neutronphysics, } \\
\text { KTH, Stockholm } \\
\text { (scientist: U.Dahlborg) }\end{array}$ \\
\hline H5 & Triple-axis spectrometer & $\begin{array}{l}\text { Department of Neutron Research, } \\
\text { UU, Studsvik (scientist: K.Sköld) }\end{array}$ \\
\hline $\mathrm{H} 6$ & $\begin{array}{l}\text { Diffractometer for protein } \\
\text { studies } \\
\text { (operational winter 1990) }\end{array}$ & $\begin{array}{l}\text { NFL } \\
\text { (scientist: L.Sjölin, CTH) }\end{array}$ \\
\hline $\mathrm{H} 7$ & $\begin{array}{l}\text { Diffractometer for } \\
\text { disordered structures } \\
\text { (operational winter 1990) }\end{array}$ & $\begin{array}{l}\text { NFL } \\
\text { (scientist: R.Delaplane) }\end{array}$ \\
\hline $\mathrm{H} 8$ & Powder diffractometer & $\begin{array}{l}\text { Department of Chemistry, UU } \\
\text { (scientist: R.Tellgren) }\end{array}$ \\
\hline H9 & $\begin{array}{l}\text { Single-crystal diffractometer } \\
\text { (operational but temporarally } \\
\text { placed at beam tube } \mathrm{H} 3 \text { ) }\end{array}$ & $\begin{array}{l}\text { Department of Chemistry, UU } \\
\text { (scientist: R.Tellgren) }\end{array}$ \\
\hline $\mathrm{H} 10$ & $\begin{array}{l}\text { Time-of-flight spectrometer } \\
\text { for cold neutrons } \\
\text { (operational winter 1991) }\end{array}$ & $\begin{array}{l}\text { Department of Neutronphysics, } \\
\text { KTH, and NFL } \\
\text { (scientists: U.Dahlborg and K.Sköld) }\end{array}$ \\
\hline $\mathrm{H} 10 \mathrm{~A}$ & $\begin{array}{l}\text { Spectrometer for polarized } \\
\text { neutrons } \\
\text { (operational winter 1991) }\end{array}$ & $\begin{array}{l}\text { Department of Neutronphysics, } \\
\text { KTH, and NFL } \\
\text { (scientist: U.Dahlborg) }\end{array}$ \\
\hline
\end{tabular}

KTH: Royal Institute of Technology, Stockholm; tel. 08/7906396

UU: Uppsala University; tel. 018/183776

CTH: Chalmers University, Gothenburg; tel. 031/722856

NFL: Neutron Research Laboratory, Studsvik; tel. 0155/21852 (UD), 21843 (RD), 21849 (KS) 


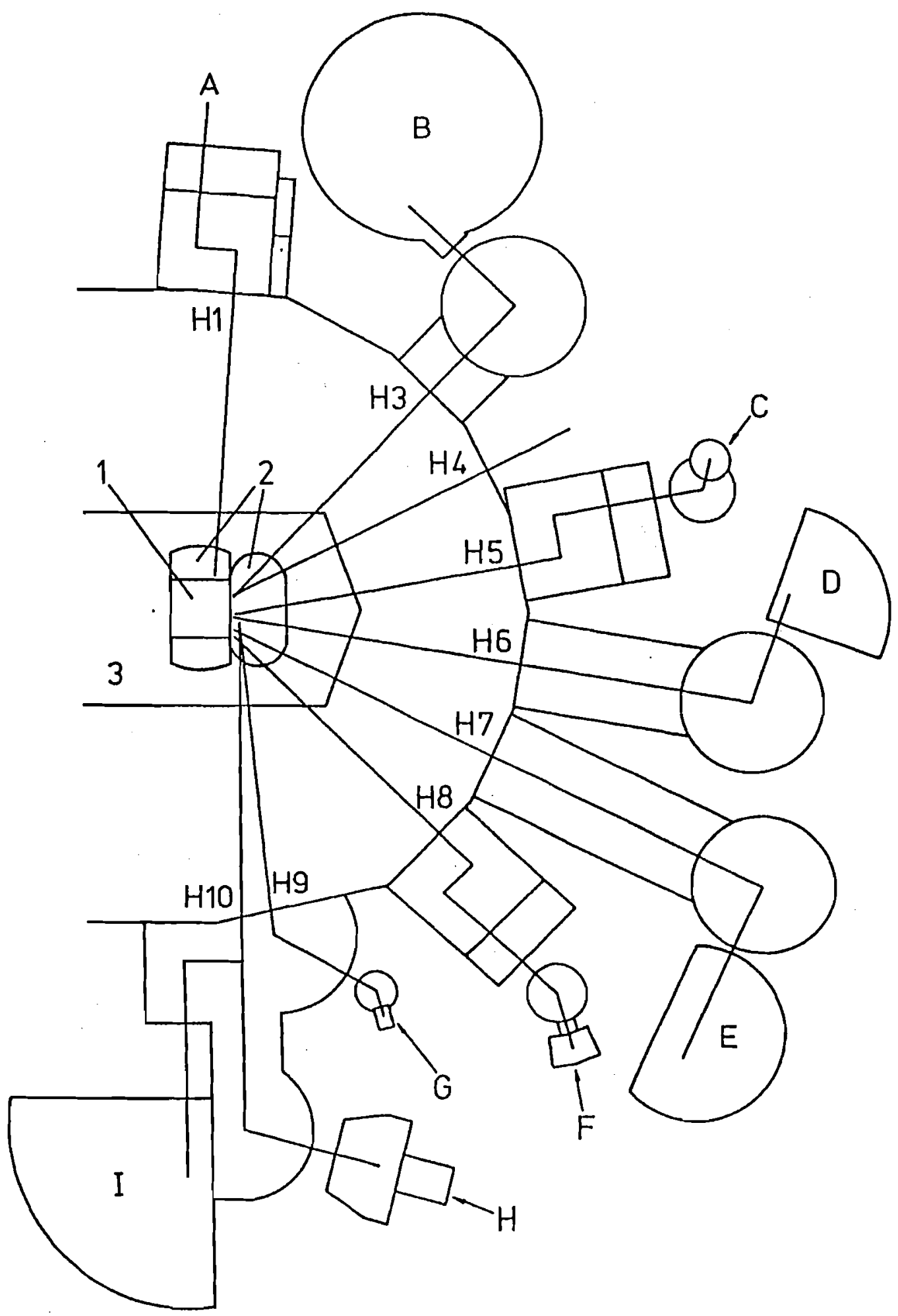

Diagram shows layout of the instrumentation around R2, Studsvik.

1) Reactor core (enriched ${ }^{235} \mathrm{U}$ and $\mathrm{H}_{2} \mathrm{O}$ ); 2) $\mathrm{D}_{2} \mathrm{O}$ moderator; 3) reactor pool 


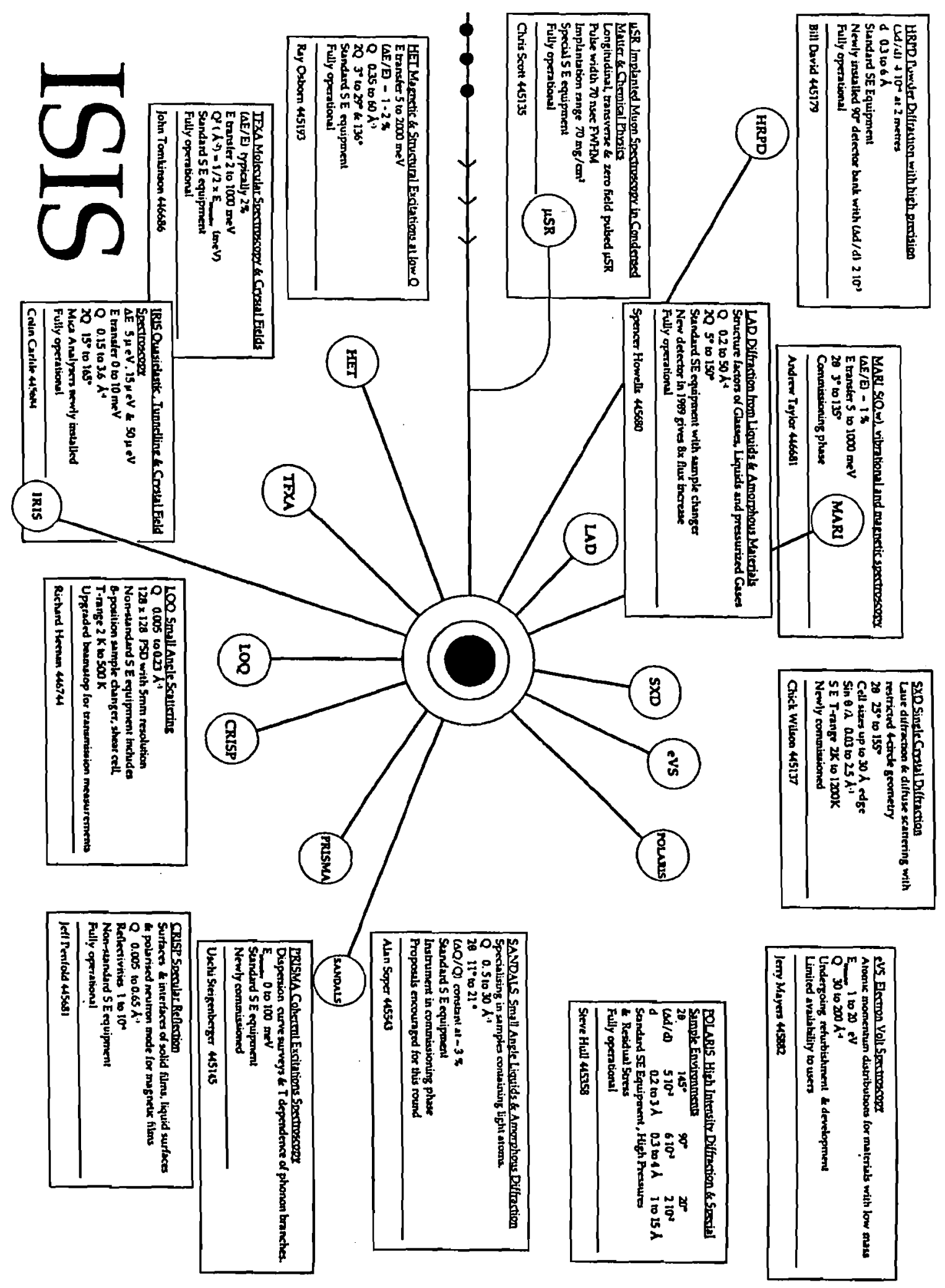

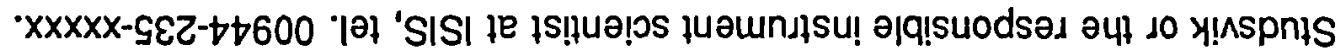

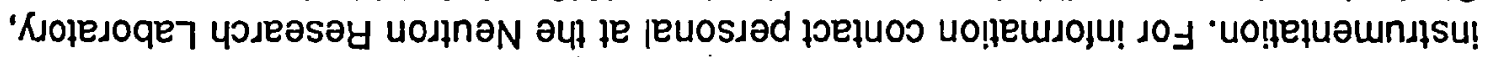

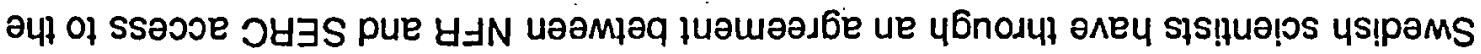

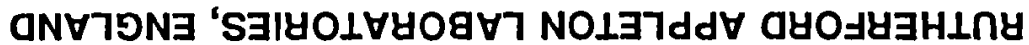

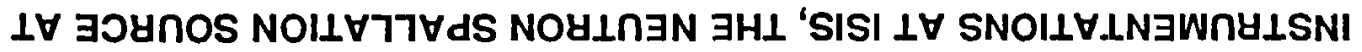
¿ xppuədd $\forall$ 


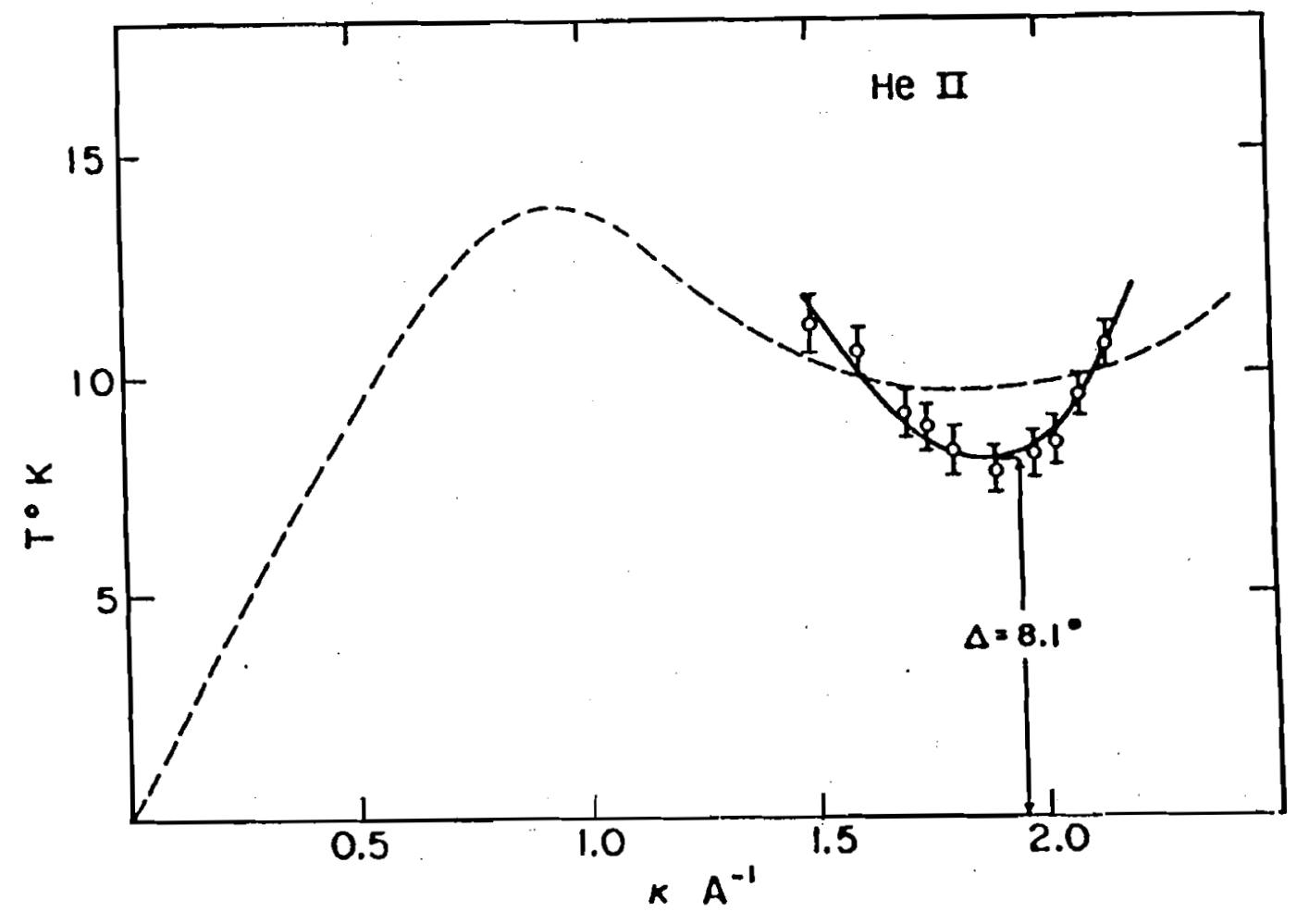

Fig. I 
$2 . \underline{b_{2}}$
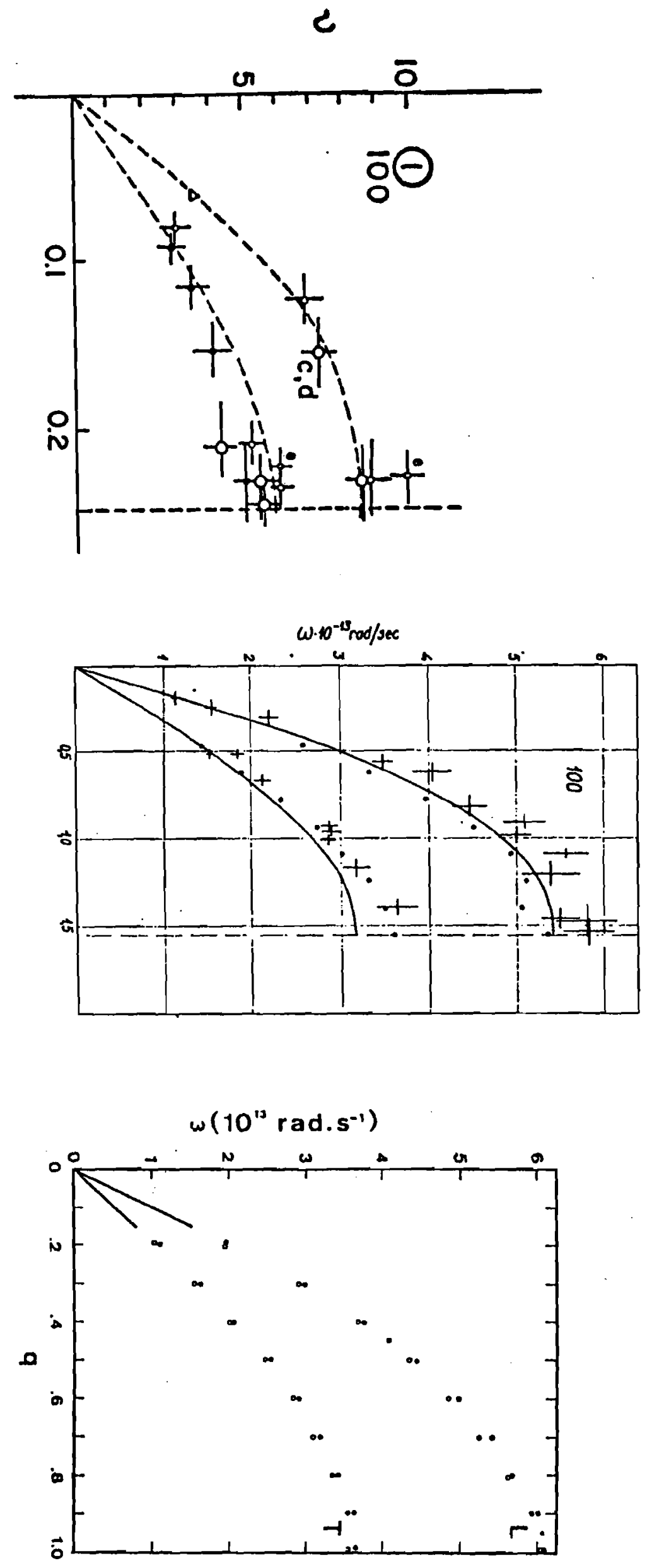

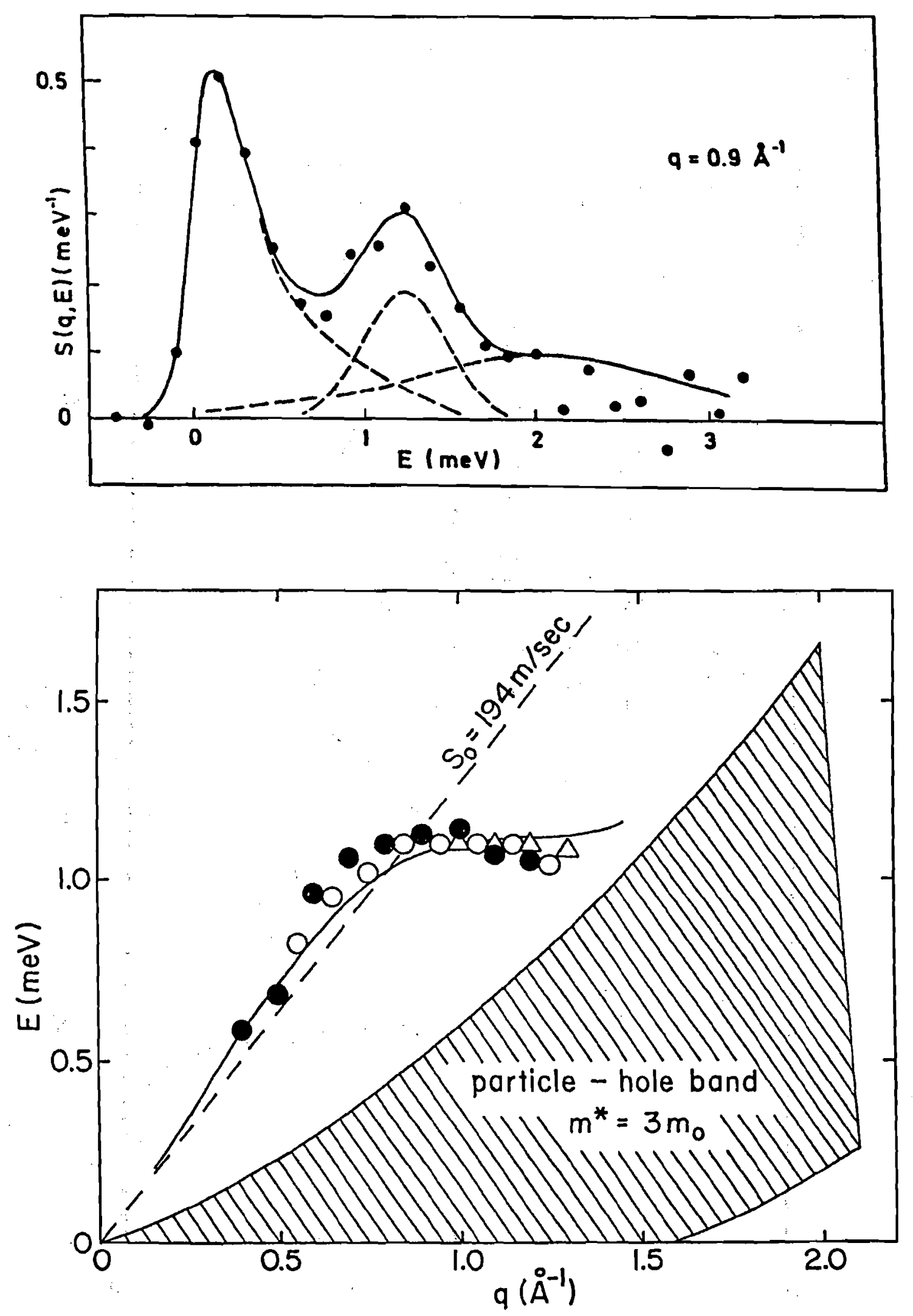

$$
\text { Tigs }
$$




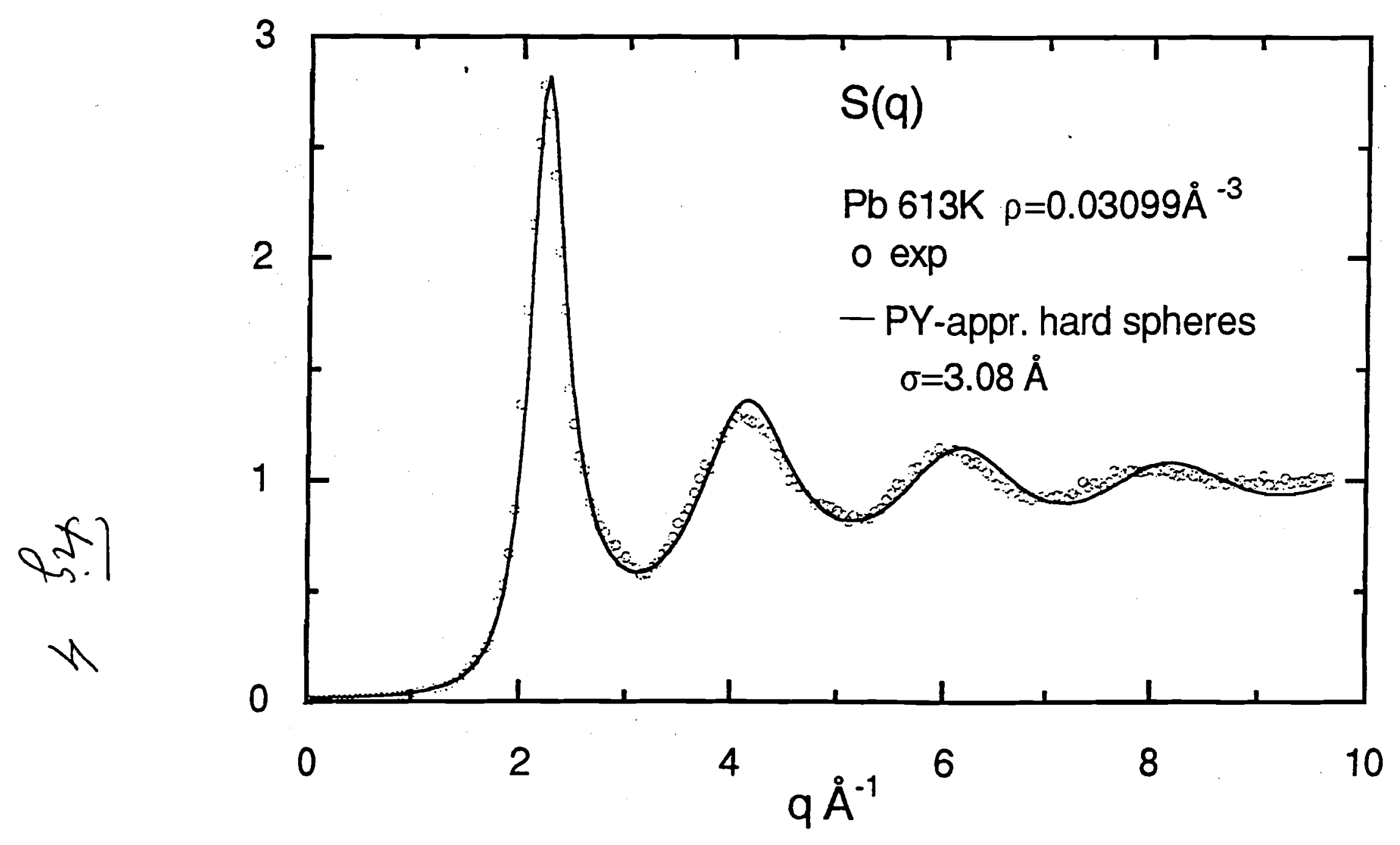




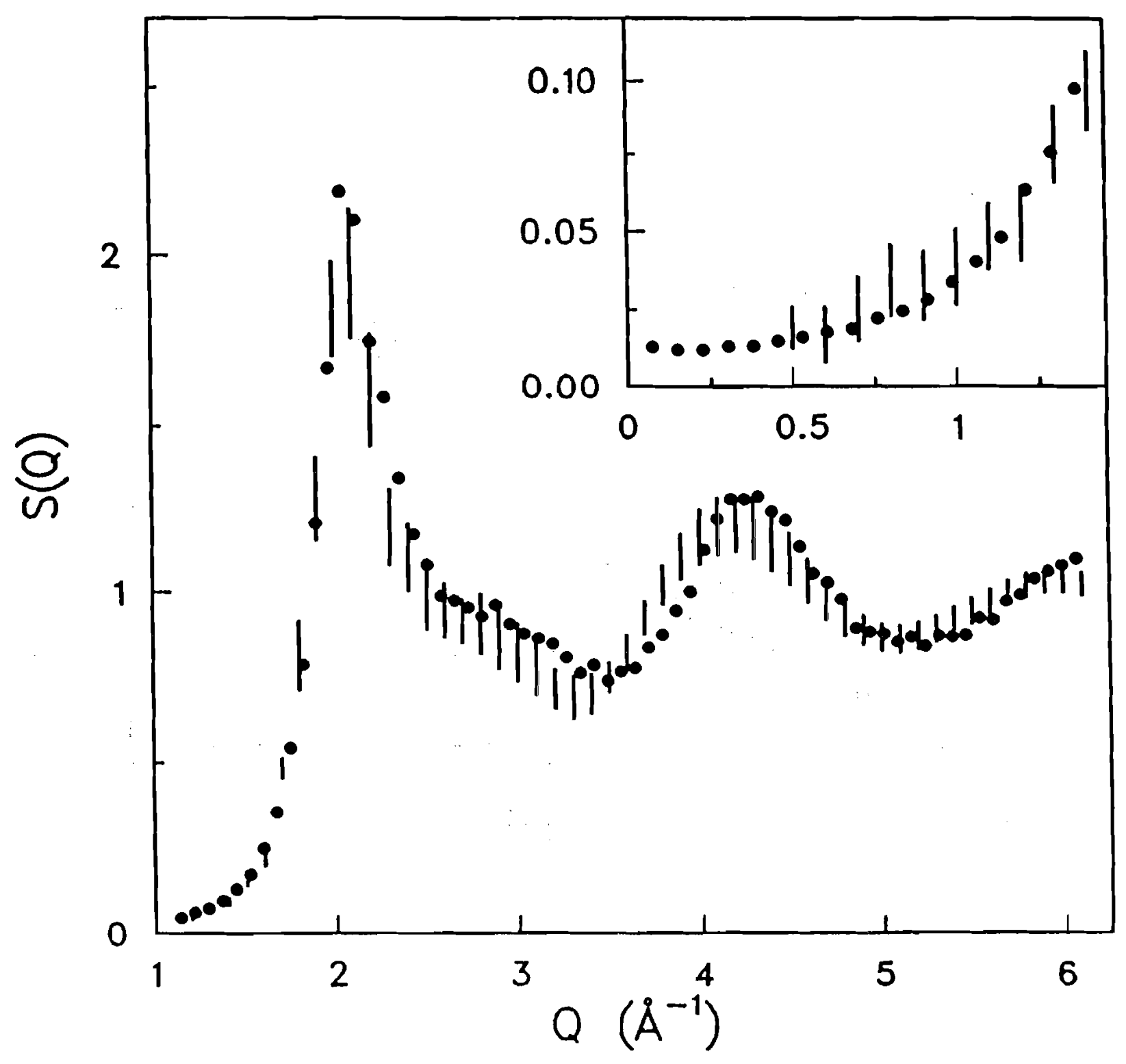

Fig 5 
9 b.

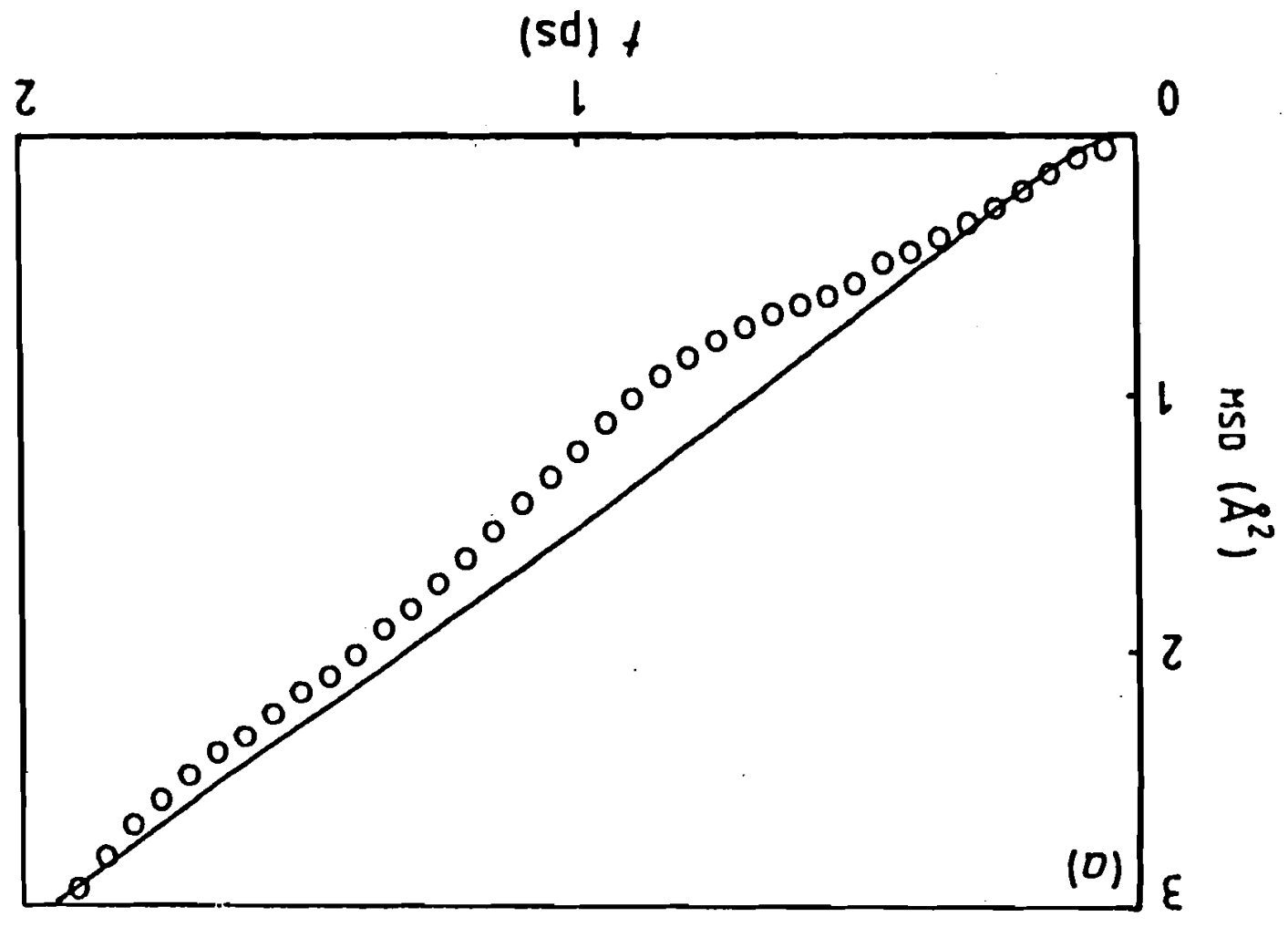



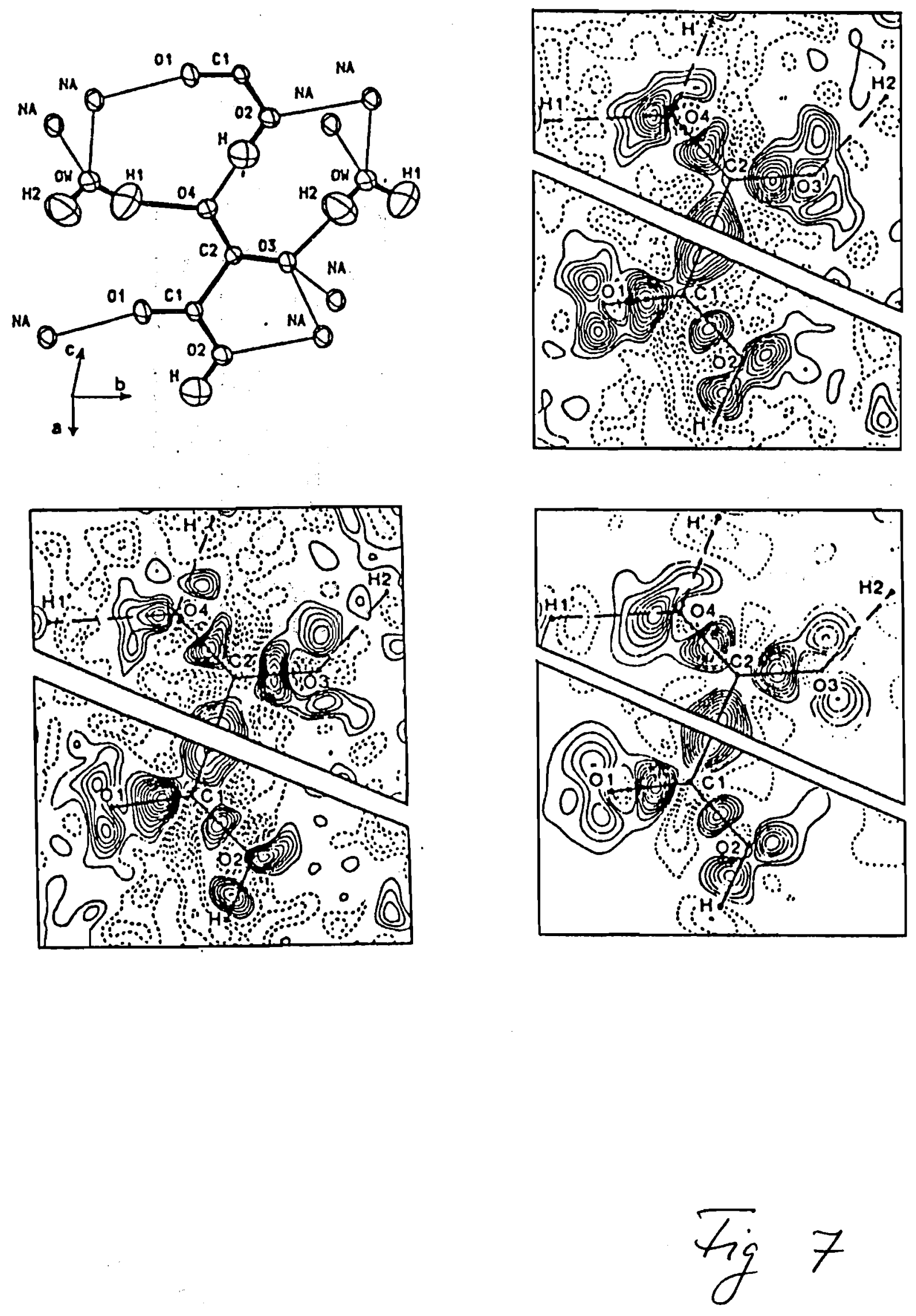
$8 \underline{b}$
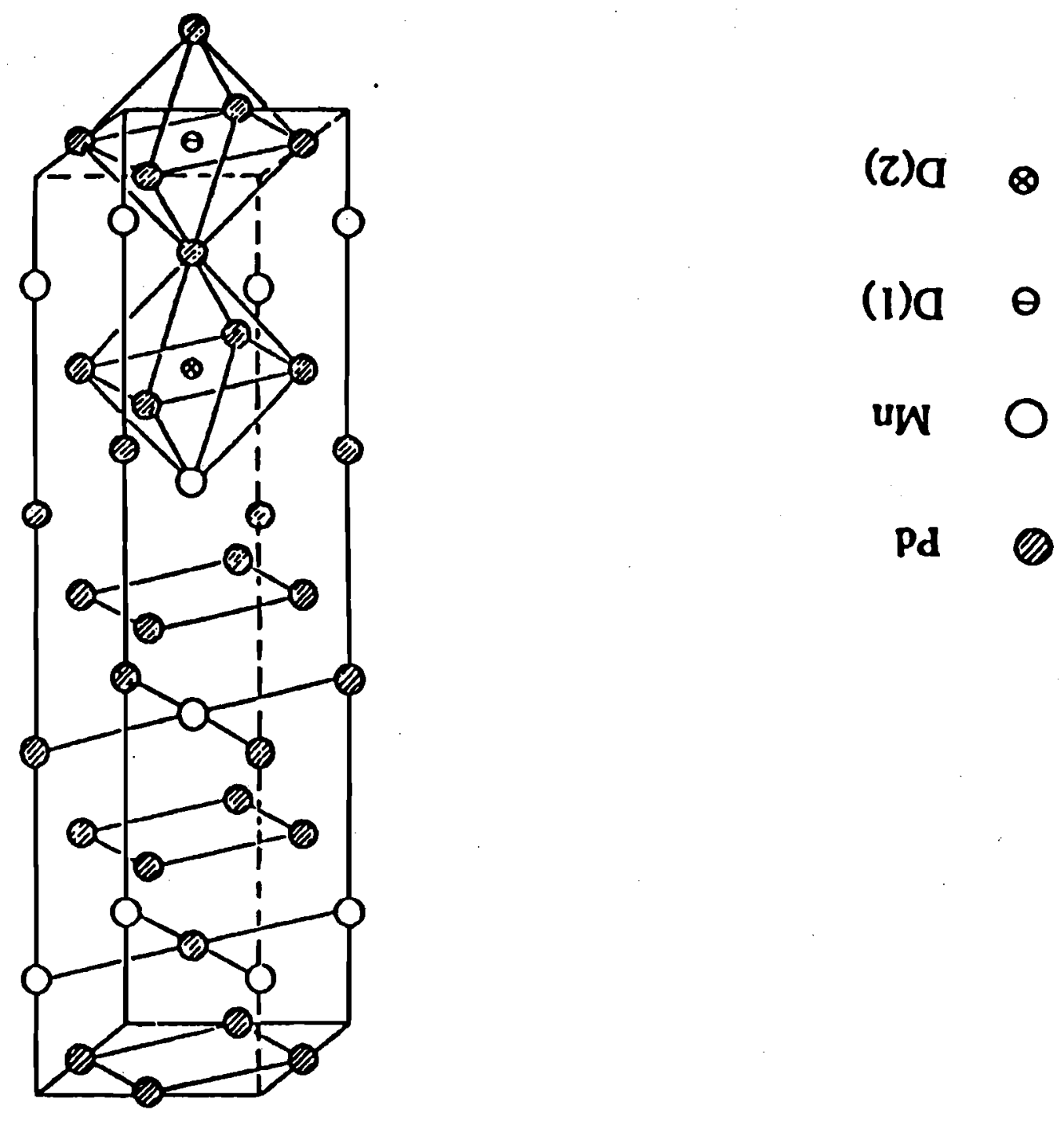


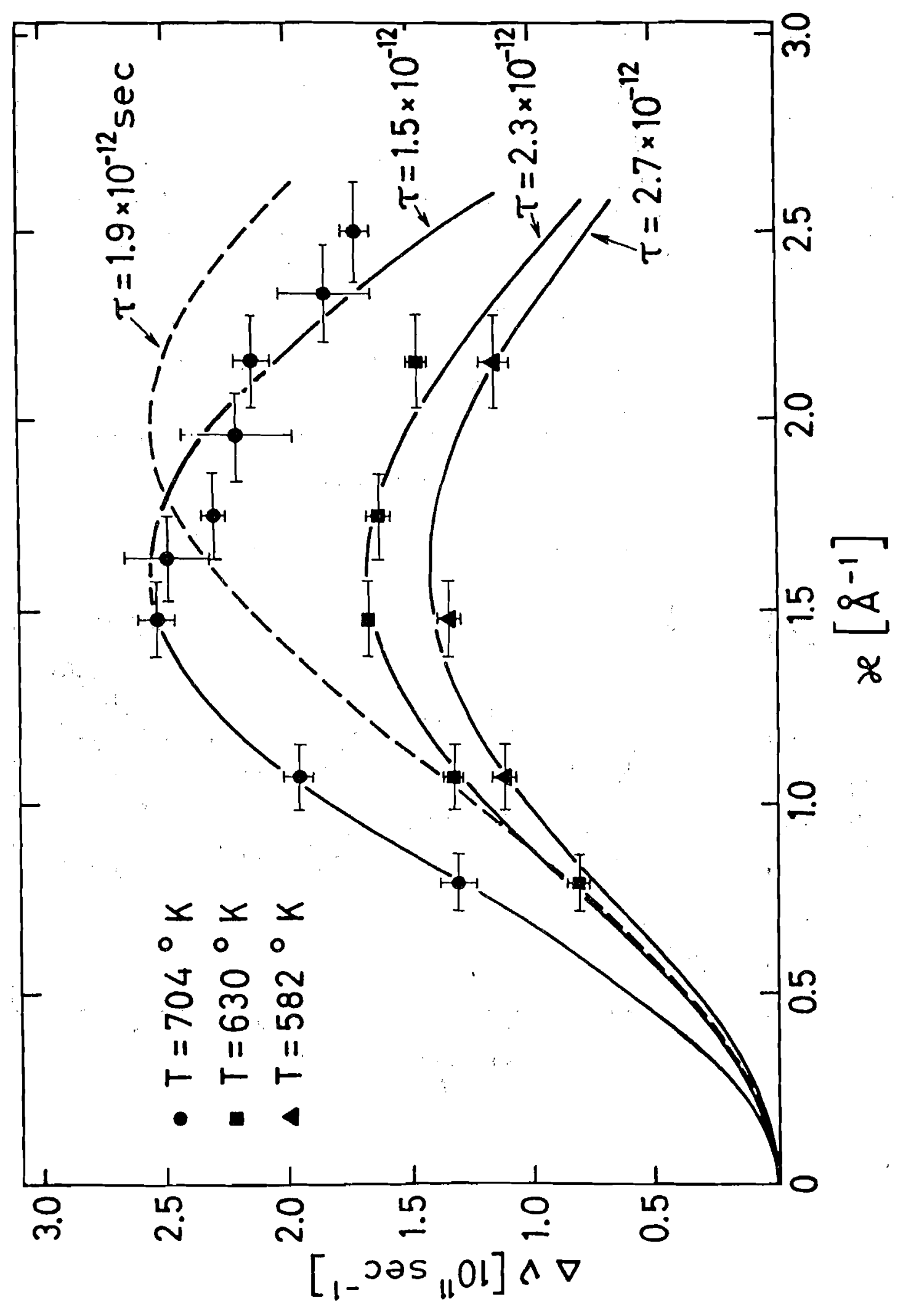

Fig 9 
0.54

(q)

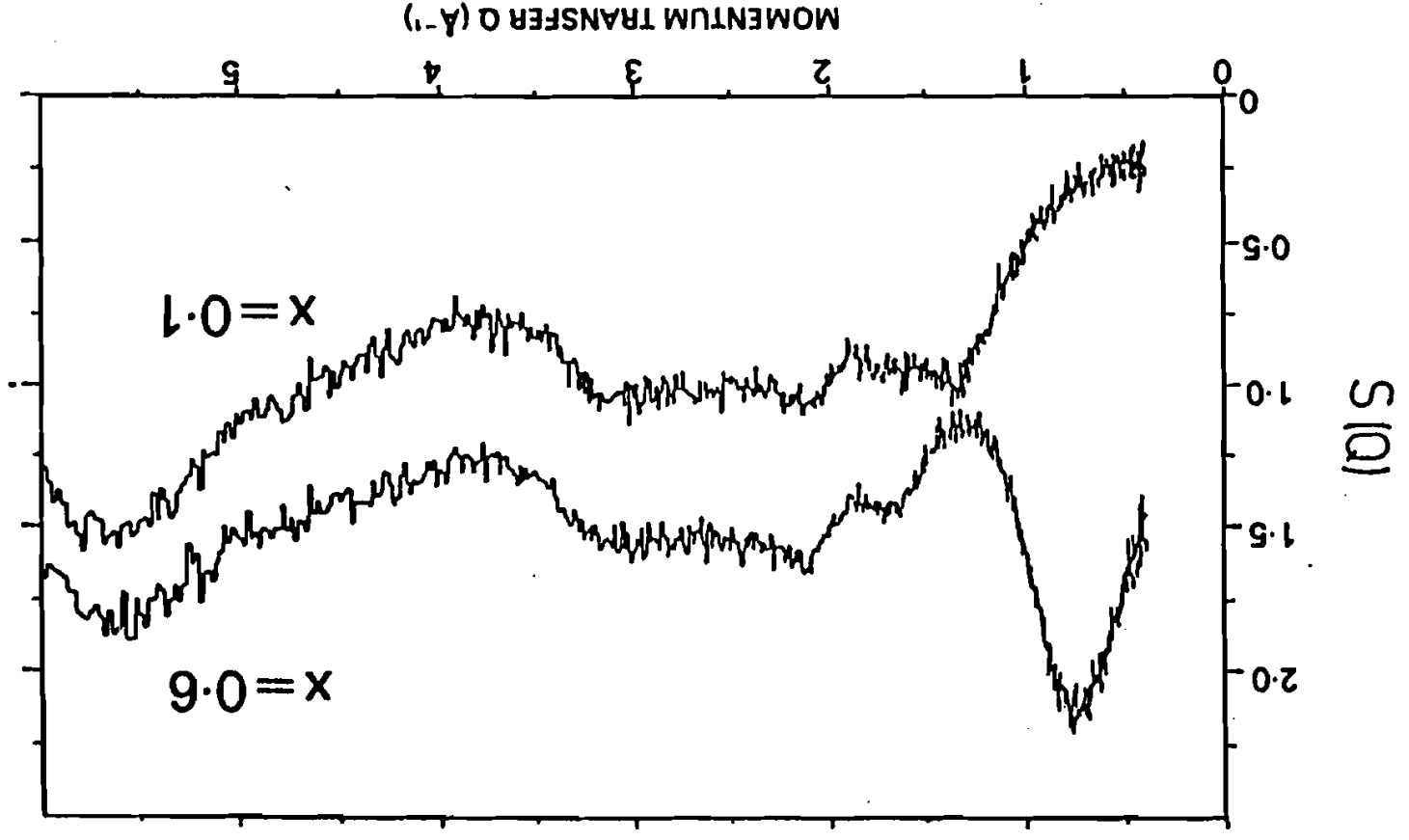

(b)

$(1-Y)$ O YGASNYYI WNLNJWOW

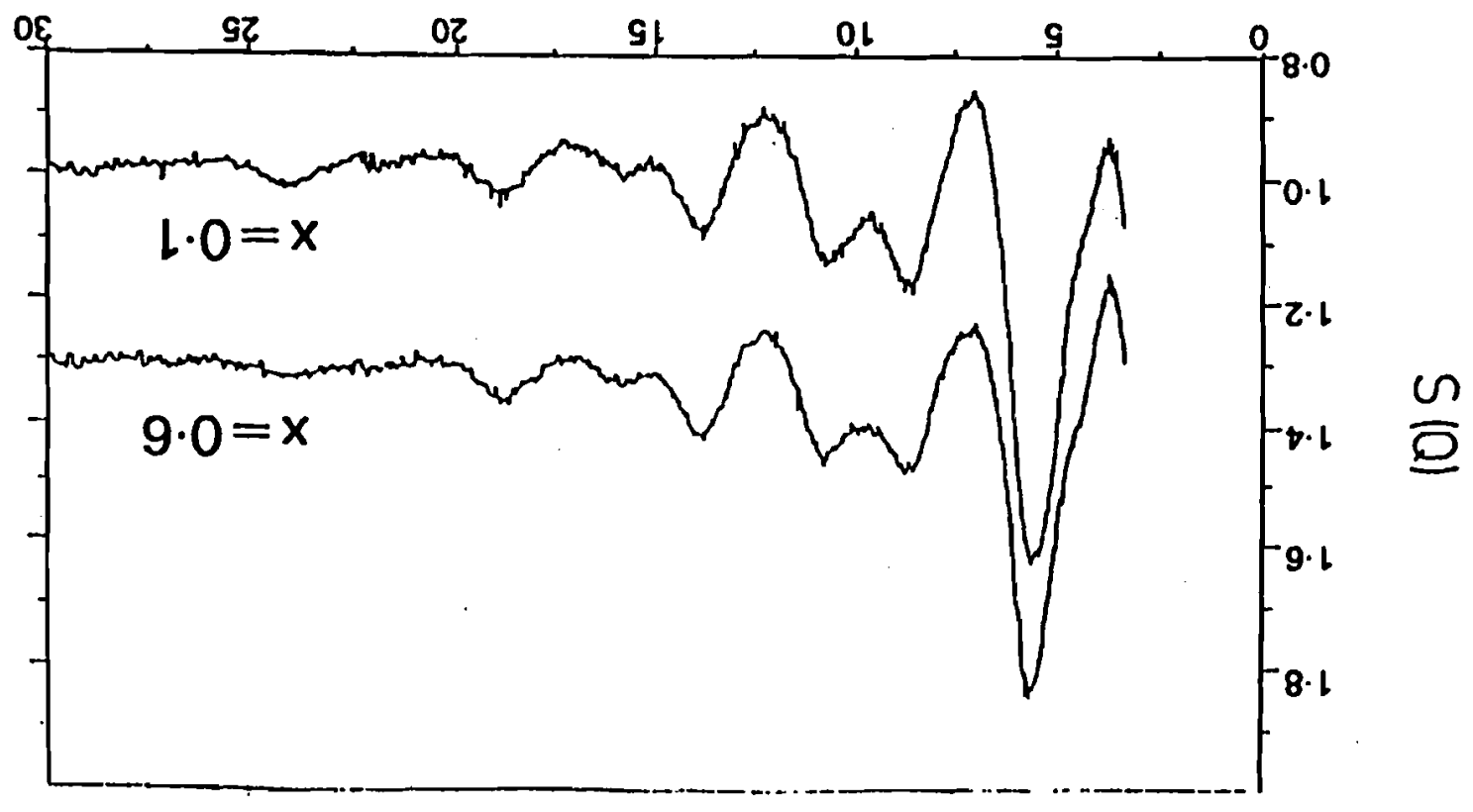




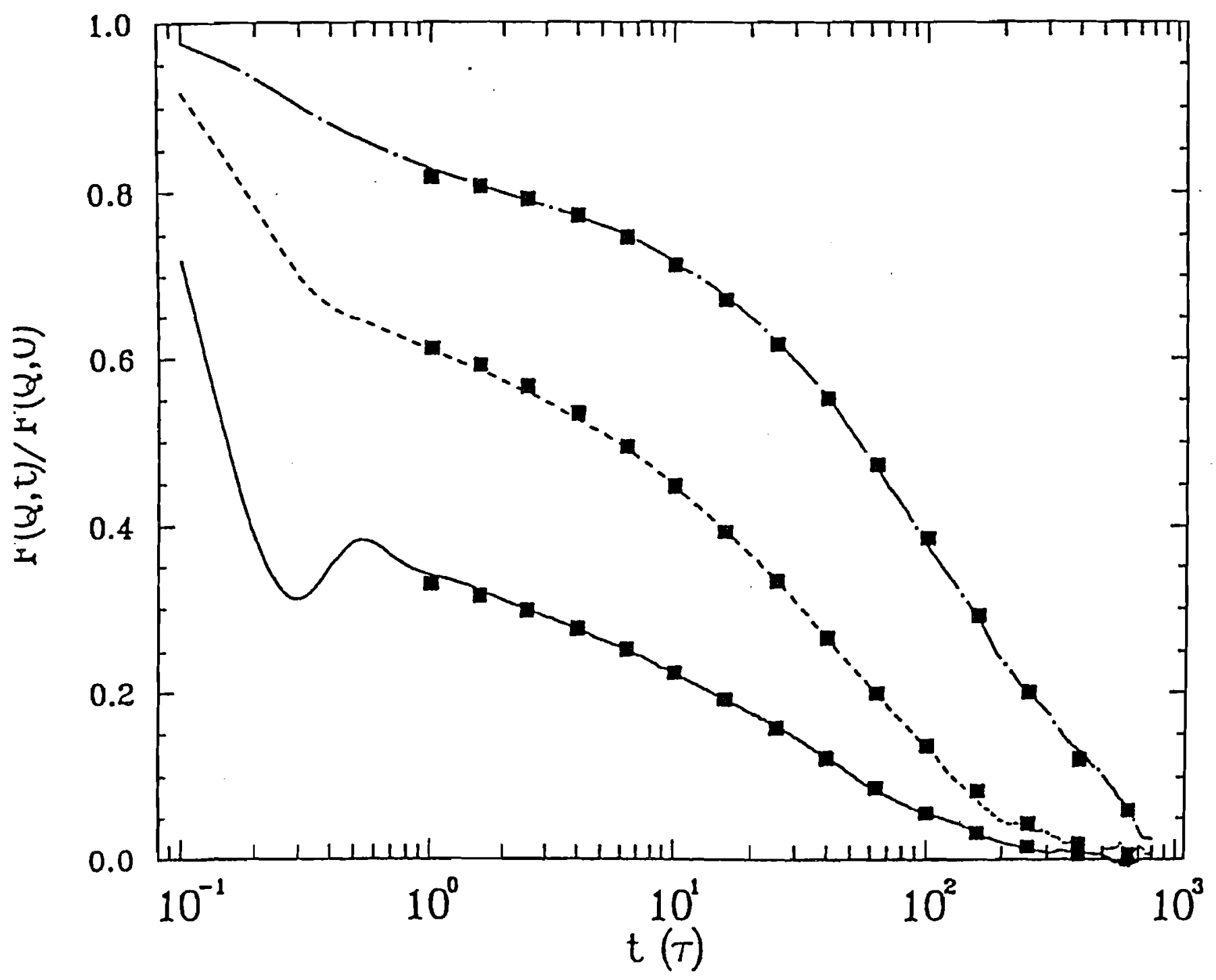

Fij I 


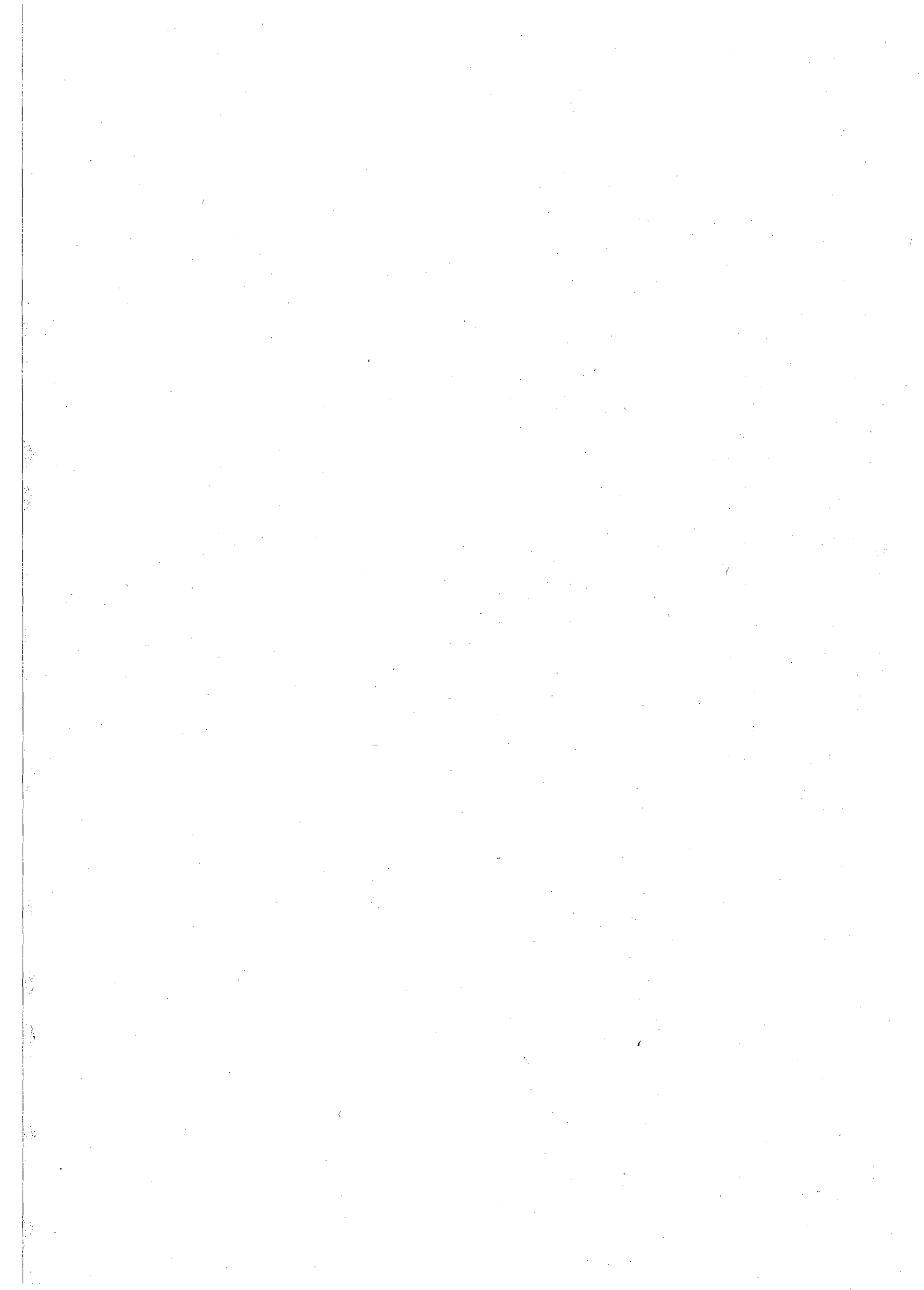


